\title{
Using Chlorotrifluoroethane for Tifluoroethylation of (Hetero)Aryl Bromides and Chlorides via Nickel Catalysis
}

\author{
Xuefei Li, ${ }^{1}$ Xing Gao ${ }^{1}$, Chun-Yang He, ${ }^{2}$ and Xingang Zhang*,1
}

${ }^{1}$ Key Laboratory of Organofluorine Chemistry, Center for Excellence in Molecular Synthesis, Shanghai Institute of Organic Chemistry, University of Chinese Academy of Sciences, Chinese Academy of Sciences, 345 Lingling Lu, Shanghai 200032, China

${ }^{2}$ Key Laboratory of Biocatalysis \& Chiral Drug Synthesis of Guizhou Province, Generic Drug Research Center of Guizhou Province, Zunyi Medical University, Zunyi, Guizhou, 563003, P. R. China 


\section{Table of Contents}

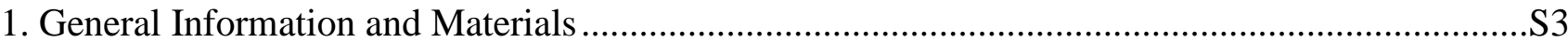

2. Optimization of the Nickel-Catalyzed Cross-Coupling of $\mathrm{CF}_{3} \mathrm{CH}_{2} \mathrm{Cl}$ with Phenylbromide or Phenylchloride (Tables S1-S11).

3. General Procedure for the Nickel-Catalyzed Cross-Coupling of $\mathrm{CF}_{3} \mathrm{CH}_{2} \mathrm{Cl}$ with (Hetero)aryl Bromides and Chlorides

4. Characterization Data of Compounds 3-35e.................................................................................

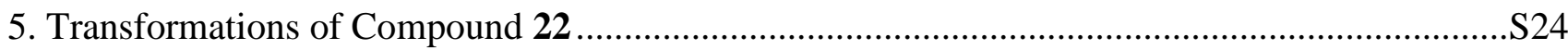

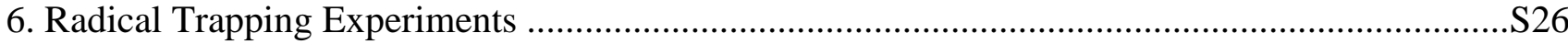

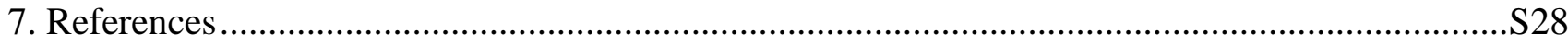

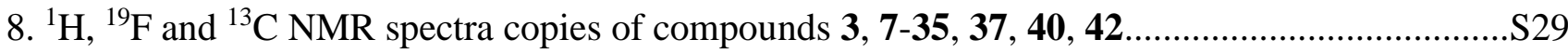




\section{General Information and Materials}

General information: ${ }^{1} \mathrm{H}$ NMR and ${ }^{13} \mathrm{C}$ NMR spectra were recorded on a Bruker AM400 and AM500 spectrometer. ${ }^{19} \mathrm{~F}$ NMR was recorded on a Bruker $\mathrm{AM} 400$ spectrometer $\left(\mathrm{CFCl}_{3}\right.$ as an external standard and low field is positive). Chemical shifts $(\delta)$ are reported in ppm, and coupling constants $(J)$ are in Hertz $(\mathrm{Hz})$. The following abbreviations were used to explain the multiplicities: $\mathrm{s}$ $=$ singlet, $\mathrm{d}=$ doublet, $\mathrm{t}=$ triplet, $\mathrm{q}=$ quartet, $\mathrm{m}=$ multiplet, $\mathrm{br}=$ broad. NMR yield was determined by ${ }^{19} \mathrm{~F}$ NMR using fluorobenzene as an internal standard before working up the reaction.

Materials: All reagents, including compounds L1-L12, 1, 2, 6, 34a-c, indomethacin, 34e, 36 and 39 were used as received from commercial sources. Compound 1 was purchased from Qinba Chemie (http://www.qb-chemie.com) and stored in a high-pressure cylinder. Compounds L1-L12, 2, 6, 34a-c, indomethacin, 34e, 36, 39, catalysts and solvents were purchased from three suppliers: Sigma Aldrich $^{\circledR}, \mathrm{TCI}^{\circledR}$ and Energy Chemical (https://www.energy-chemical.com). Compound 34d was synthesized from indomethacin according to the literature, ${ }^{1}$ and its spectroscopic characterizations were consistent with the literature. ${ }^{1} \mathrm{DMF}, \mathrm{DMA}, \mathrm{CH}_{3} \mathrm{CN}$ and DMPU were distilled under reduced pressure from $\mathrm{CaH}_{2}$. 1, 4-dioxane and THF were distilled from sodium and benzophenone immediately before use, all solvents were degassed by argon. Zinc powder was activated by aqueous hydrochloric acid solutions (1 M) before used.

\section{Preparation of $\mathrm{CF}_{3} \mathrm{CH}_{2} \mathrm{Cl}$ stock solution:}

The solubility of $\mathrm{CF}_{3} \mathrm{CH}_{2} \mathrm{Cl}$ in common solvents is good, we take DMF as an example. Under argon atmosphere, to a $25 \mathrm{~mL}$ Schlenk tube was added DMF $(22 \mathrm{~mL})$, then $\mathrm{CF}_{3} \mathrm{CH}_{2} \mathrm{Cl}$ was slowly bubbled using a long needle until the total volume of the solution reached approximately $25 \mathrm{~mL}$. Then, the tube was sealed with a Teflon-lined cap. The concentration of the $\mathrm{CF}_{3} \mathrm{CH}_{2} \mathrm{Cl}$ stock solution (2 M) was determined by ${ }^{19}$ F NMR using fluorobenzene as an internal standard. 
2. Optimization of the Nickel-Catalyzed Cross-Coupling of $\mathrm{CF}_{3} \mathrm{CH}_{2} \mathrm{Cl}$ with Phenylbromide or Phenylchloride (Tables S1-S11).

\section{Screenings for nickel-catalyzed cross-coupling of $\mathrm{CF}_{3} \mathrm{CH}_{2} \mathrm{Cl} 1$ with bromobenzene $2 \mathrm{a}$ (Tables}

S1-S10). To a $25 \mathrm{~mL}$ of Schlenk tube were added manganese or zinc powder (1.8 - 3.4 equiv) and ligand (0.02 - 0.12 equiv). The tube was evacuated and back-filled with argon for three times, then nickel catalyst (0.02 - 0.12 equiv), additive ( $0.2-1.25$ equiv), bromobenzene 2a (1.0 - 1.8 equiv), anhydrous solution of $\mathrm{CF}_{3} \mathrm{CH}_{2} \mathrm{Cl} 1$ ( $0.2 \mathrm{mmol}, 1.0$ equiv), and solvent $(2 \mathrm{~mL})$ were added. The reaction mixture was heated to $50-90{ }^{\circ} \mathrm{C}$ (oil bath). After stirring for 6 - 24 hours at $50-90{ }^{\circ} \mathrm{C}$, the reaction mixture was cooled to room temperature and quenched with water $(2 \mathrm{~mL})$ and ethyl acetate $(2 \mathrm{~mL})$. The yield was determined by ${ }^{19} \mathrm{~F}$ NMR using fluorobenzene as an internal standard.

Table S1. Screening of the nickel sources ${ }^{a}$

\begin{tabular}{|c|c|c|c|c|c|}
\hline $\mathrm{Cl}^{-}$ & $\overbrace{2 a}^{B r}$ & $\begin{array}{c}\text { [Ni] }(10 \mathrm{~mol} \%) \\
\text { 4,4'- ditBu-bpy (10 mol\%) } \\
\text { Mn (3.0 equiv) } \\
\text { DMF, } 80^{\circ} \mathrm{C}, 12 \mathrm{~h}\end{array}$ & & $\mathrm{CF}_{3}$ & \\
\hline \multirow{2}{*}{ Entry } & \multirow{2}{*}[\mathrm{Ni}]{} & \multirow{2}{*}{ 1, Conversion $(\%)$} & \multicolumn{3}{|c|}{ Yield $(\%)^{b}$} \\
\hline & & & 3 & 4 & 5 \\
\hline 1 & $\mathrm{NiBr}_{2} \cdot 3 \mathrm{H}_{2} \mathrm{O}$ & 26 & 12 & 0 & 0 \\
\hline 2 & $\mathrm{NiBr}_{2}$ & 55 & 27 & 3 & $\mathbf{0}$ \\
\hline 3 & $\mathrm{NiI}_{2}$ & 55 & 20 & 4 & 0 \\
\hline 4 & $\mathrm{NiBr}_{2} \cdot\left(\mathrm{PPh}_{3}\right)_{2}$ & 22 & 13 & 0 & 0 \\
\hline 5 & $\mathrm{NiCl}_{2} \cdot\left(\mathrm{PPh}_{3}\right)_{2}$ & 40 & 17 & 1 & 0 \\
\hline 6 & $\mathrm{Ni}(\mathrm{COD})_{2}$ & 39 & 24 & 0 & 0 \\
\hline 7 & $\mathrm{NiCl}_{2} \cdot \mathrm{DME}$ & 53 & 24 & 4 & 0 \\
\hline 8 & $\mathrm{NiBr}_{2} \cdot \mathrm{DME}$ & 74 & 26 & 5 & 0 \\
\hline 9 & $\mathrm{NiBr}_{2} \cdot$ glyme & 59 & 27 & 5 & 0 \\
\hline
\end{tabular}

${ }^{a}$ Reaction conditions (unless otherwise specified): 1 ( 0.2 mmol, 1.0 equiv), 2a (1.5 equiv), DMF (2 $\mathrm{mL}) .{ }^{b}$ Determined by ${ }^{19} \mathrm{~F}$ NMR using fluorobenzene as an internal standard. 
Table S2. Screening of the ligands ${ }^{a}$
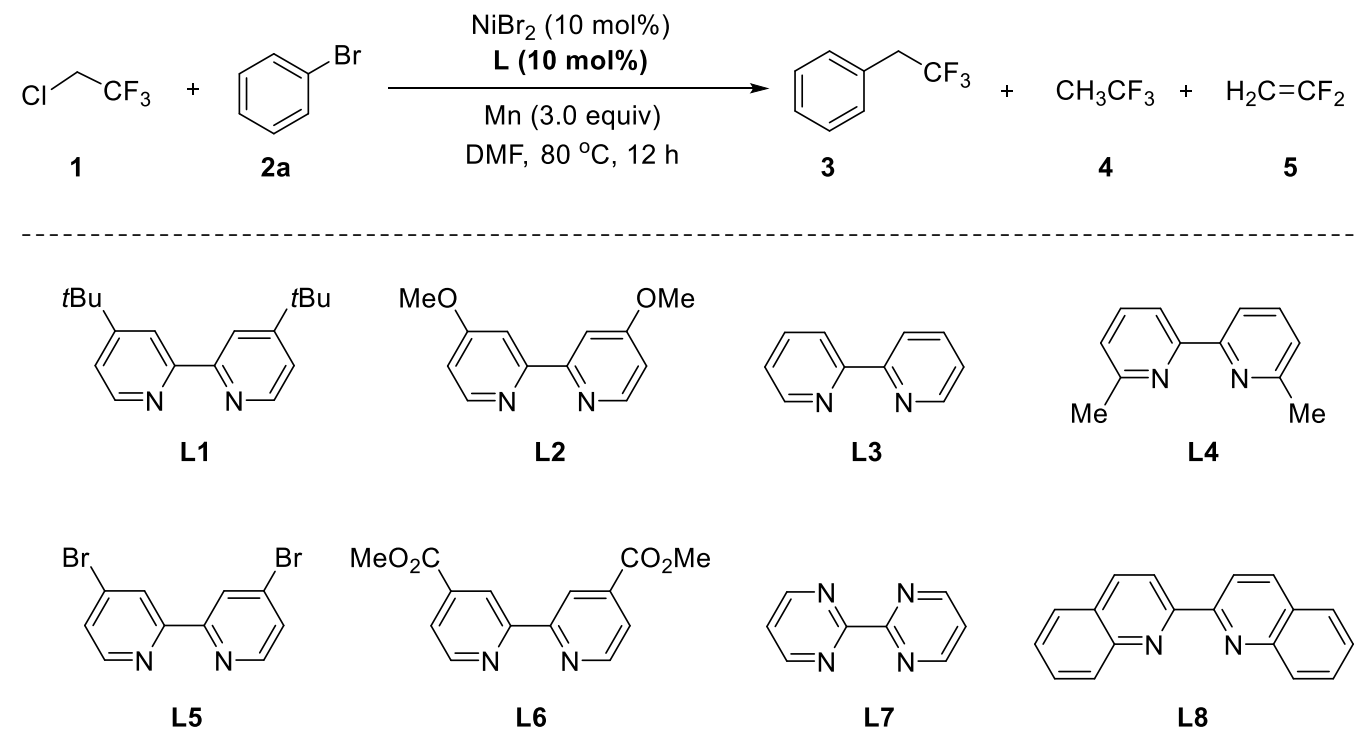

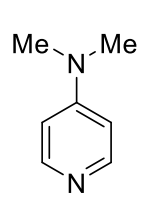

L9

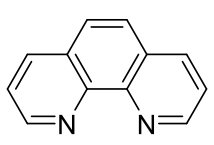

L10

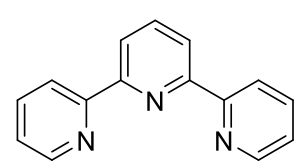

L11

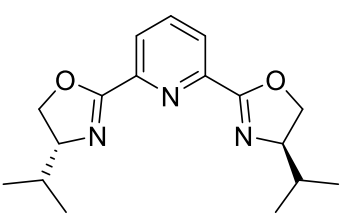

L12

\begin{tabular}{cccccc}
\hline \multirow{2}{*}{ Entry } & Ligand $(\mathbf{L})$ & $\mathbf{1}$, Conversion $(\%)$ & $\mathbf{3}$ & $\mathbf{4}$ & $\mathbf{5}$ \\
\hline $\mathbf{1}$ & $\mathbf{L 1}$ & $\mathbf{5 5}$ & $\mathbf{2 7}$ & $\mathbf{3}$ & $\mathbf{0}$ \\
2 & $\mathbf{L 2}$ & 22 & 14 & 0 & 0 \\
3 & $\mathbf{L 3}$ & 16 & 12 & 0 & 0 \\
4 & $\mathbf{L 4}$ & 67 & 5 & 5 & 1 \\
5 & $\mathbf{L 5}$ & 8 & trace & 0 & 0 \\
6 & $\mathbf{L 6}$ & 8 & 1 & 0 & trace \\
7 & $\mathbf{L 7}$ & 25 & 2 & 0 & 0 \\
8 & $\mathbf{L 8}$ & 20 & 0 & 0 & 0 \\
9 & $\mathbf{L 9}$ & 13 & 1 & 3 & 0 \\
10 & $\mathbf{L 1 0}$ & 67 & 26 & 5 & 1 \\
11 & $\mathbf{L 1 1}$ & 100 & 12 & 9 & 9 \\
12 & $\mathbf{L 1 2}$ & 79 & 5 & 10 & 3 \\
\hline
\end{tabular}

${ }^{a}$ Reaction conditions (unless otherwise specified): $\mathbf{1}$ ( 0.2 mmol, 1.0 equiv), 2 a (1.5 equiv), DMF (2 $\mathrm{mL}) .{ }^{b}$ Determined by ${ }^{19} \mathrm{~F}$ NMR using fluorobenzene as an internal standard. 
Table S3. Screening of the solvents ${ }^{a}$

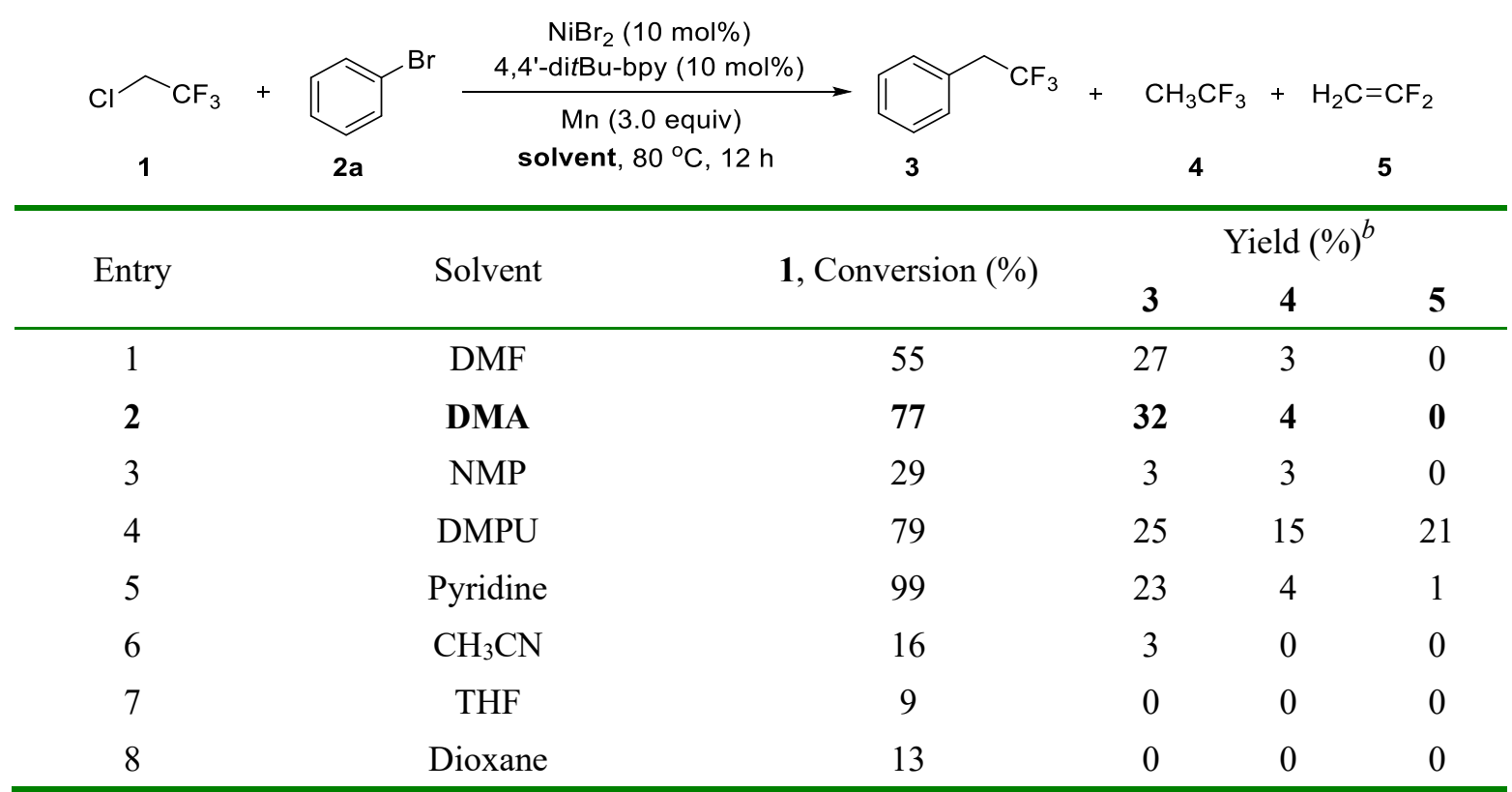

${ }^{a}$ Reaction conditions (unless otherwise specified): 1 ( $0.2 \mathrm{mmol}, 1.0$ equiv), 2a ( 1.5 equiv), solvent ( 2 $\mathrm{mL}) .{ }^{b}$ Determined by ${ }^{19} \mathrm{~F}$ NMR using fluorobenzene as an internal standard. 
Table S4. Screening of the reductants and additives ${ }^{a}$

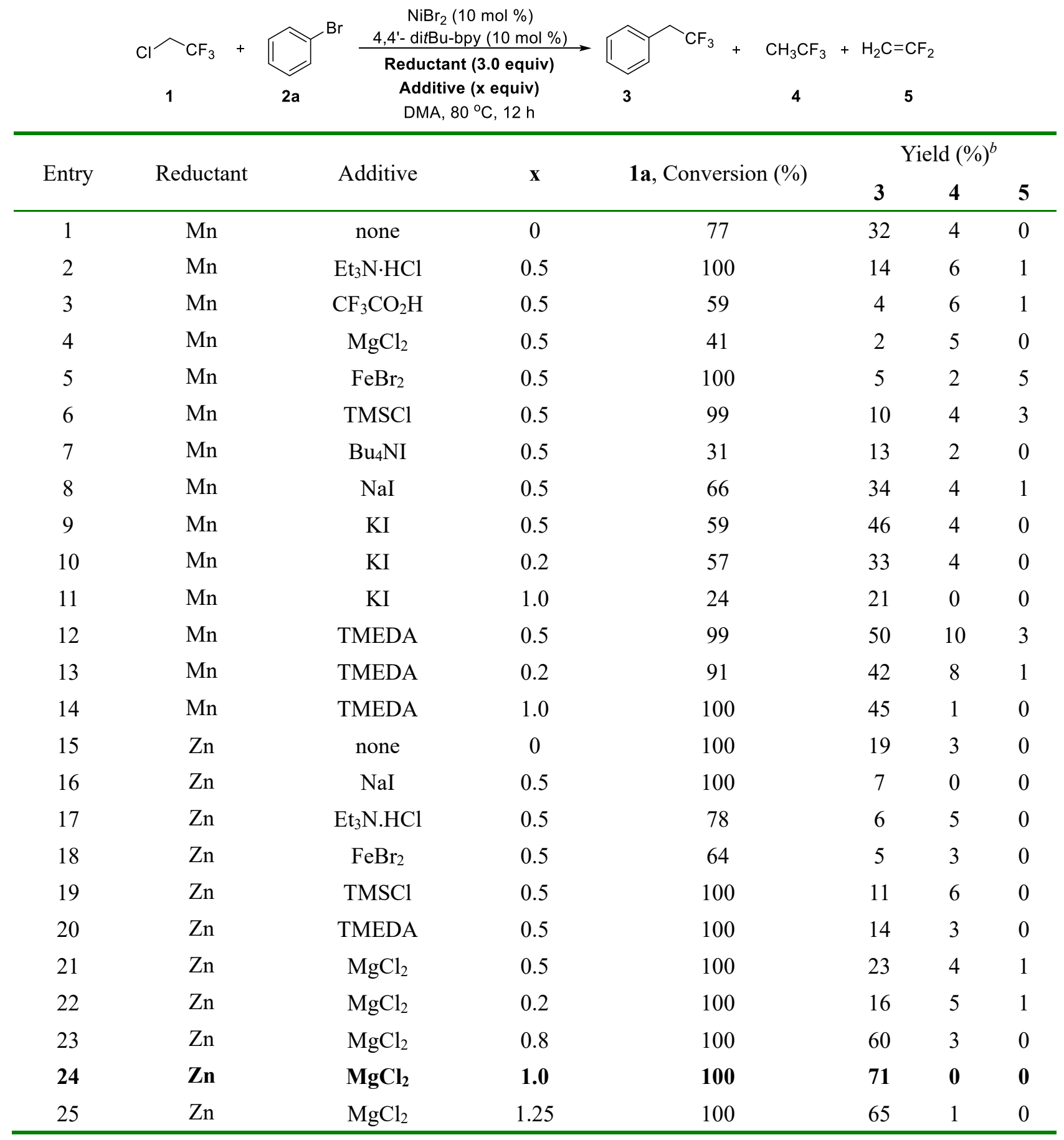

${ }^{a}$ Reaction conditions (unless otherwise specified): 1 ( 0.2 mmol, 1.0 equiv), 2a (1.5 equiv), DMA (2 $\mathrm{mL}) .{ }^{b}$ Determined by ${ }^{19} \mathrm{~F}$ NMR using fluorobenzene as an internal standard. 
Table S5. Screening of the magnesium salts and metal chlorides ${ }^{a}$

\begin{tabular}{|c|c|c|c|c|c|}
\hline & $2 a$ & $\begin{array}{c}\mathrm{NiBr}_{2}(10 \mathrm{~mol} \%) \\
4,4^{\prime}-\text { ditBu-bpy }(10 \mathrm{~mol} \%) \\
\mathrm{Zn}(3.0 \text { equiv) } \\
\text { Additive }(1.0 \text { equiv) } \\
\text { DMA, } 80^{\circ} \mathrm{C}, 12 \mathrm{~h}\end{array}$ & & & \\
\hline \multirow{2}{*}{ Entry } & \multirow{2}{*}{ Additives } & \multirow{2}{*}{ 1, Conversion $(\%)$} & \multicolumn{3}{|c|}{ Yield $(\%)^{b}$} \\
\hline & & & 3 & 4 & 5 \\
\hline 1 & $\mathrm{MgCl}_{2}$ & 100 & 71 & $\mathbf{0}$ & $\mathbf{0}$ \\
\hline 2 & $\operatorname{Mg}(\mathrm{OTf})_{2}$ & 100 & 21 & 5 & 0 \\
\hline 3 & $\mathrm{MgSO}_{4}$ & 100 & 15 & 5 & 0 \\
\hline 4 & $\mathrm{MgSO}_{4} \cdot 7 \mathrm{H}_{2} \mathrm{O}$ & 56 & 6 & 7 & 0 \\
\hline 5 & $\mathrm{Mg}(\mathrm{OAc})_{2}$ & 45 & 1 & 0 & 0 \\
\hline 6 & $\mathrm{LiCl}$ & 100 & 36 & 1 & 0 \\
\hline 7 & $\mathrm{KCl}$ & 100 & 19 & 1 & 0 \\
\hline 8 & $\mathrm{CaCl}_{2}$ & 100 & 41 & 0 & 0 \\
\hline
\end{tabular}

${ }^{a}$ Reaction conditions (unless otherwise specified): 1 ( 0.2 mmol, 1.0 equiv), 2a (1.5 equiv), DMA (2 $\mathrm{mL}) .{ }^{b}$ Determined by ${ }^{19} \mathrm{~F}$ NMR using fluorobenzene as an internal standard.

Table S6. Screening of the loading amount of NiBr2 and 4, $4^{\prime}$-ditBu-bpy ${ }^{a}$

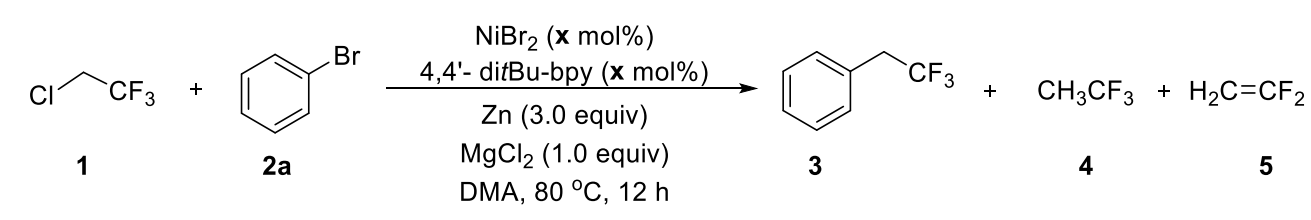

\begin{tabular}{cccccc}
\hline Entry & $\mathbf{x}$ & $\mathbf{1}$, Conversion $(\%)$ & $\mathbf{3}$ & Yield (\%) $^{\mathrm{b}}$ & $\mathbf{4}$ \\
\hline 1 & 2 & 80 & 33 & 7 & 0 \\
2 & 4 & 99 & 51 & 6 & 0 \\
3 & 6 & 100 & 56 & 3 & 0 \\
4 & 8 & 100 & 65 & 0 & 0 \\
$\mathbf{5}$ & $\mathbf{1 0}$ & $\mathbf{1 0 0}$ & $\mathbf{7 1}$ & $\mathbf{0}$ & $\mathbf{0}$ \\
6 & 12 & 100 & 72 & 1 & 0 \\
\hline
\end{tabular}

${ }^{a}$ Reaction conditions (unless otherwise specified): $\mathbf{1}\left(0.2 \mathrm{mmol}, 1.0\right.$ equiv), $\mathbf{2 a}(1.5$ equiv), DMA ( $2 \mathrm{~mL}) .{ }^{b} \mathrm{Determined} \mathrm{by}$ ${ }^{19}$ F NMR using fluorobenzene as an internal standard. 
Table S7. Screening of the reaction temperature and time ${ }^{a}$

\begin{tabular}{|c|c|c|c|c|c|c|}
\hline & $\widehat{C F}_{1}+$ & 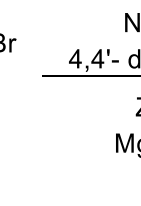 & $\begin{array}{l}\text { l\%) } \\
0 \text { mol\%) } \\
\text { iv) } \\
\text { quiv) } \\
\text { p }\end{array}$ & ${ }_{3} \mathrm{CF}_{3}$ & $\mathrm{CF}_{2}$ & \\
\hline \multirow{2}{*}{ Entry } & \multirow{2}{*}{ Temp $/{ }^{\circ} \mathrm{C}$} & \multirow{2}{*}{ Time $/ \mathrm{h}$} & \multirow{2}{*}{1, Conversion $(\%)$} & \multicolumn{3}{|c|}{ Yield $(\%)^{b}$} \\
\hline & & & & 3 & 4 & 5 \\
\hline 1 & 90 & 12 & 100 & 70 & 0 & 0 \\
\hline 2 & 80 & 12 & 100 & 71 & $\mathbf{0}$ & $\mathbf{0}$ \\
\hline 3 & 70 & 12 & 100 & 64 & 1 & 0 \\
\hline 4 & 60 & 12 & 89 & 40 & trace & 1 \\
\hline 5 & 60 & 24 & 100 & 29 & 1 & 0 \\
\hline 6 & 50 & 24 & 77 & 30 & 1 & 2 \\
\hline
\end{tabular}

${ }^{a}$ Reaction conditions (unless otherwise specified): 1 ( $0.2 \mathrm{mmol}, 1.0$ equiv), 2a (1.5 equiv), DMA (2 $\mathrm{mL}) .{ }^{b}$ Determined by ${ }^{19} \mathrm{~F}$ NMR using fluorobenzene as an internal standard.

Table S8. Screening of the ratio of 1 and $2 a^{a}$

\begin{tabular}{|c|c|c|c|c|c|}
\hline & $2 a$ & $\begin{array}{c}\mathrm{NiBr}_{2}(10 \mathrm{~mol} \%) \\
4^{4}-\text { - ditBu-bpy (10 mol\%) } \\
\mathrm{Zn}(3.0 \text { equiv) } \\
\mathrm{MgCl}_{2}(1.0 \text { equiv) } \\
\mathrm{DMA}, 80^{\circ} \mathrm{C}, 12 \mathrm{~h}\end{array}$ & & & \\
\hline \multirow{2}{*}{ Entry } & \multirow{2}{*}{ 1: $2 a$} & \multirow{2}{*}{ 1, Conversion $(\%)$} & \multicolumn{3}{|c|}{ Yield $(\%)^{b}$} \\
\hline & & & 3 & 4 & 5 \\
\hline 1 & $1: 1$ & 100 & 50 & 1 & 0 \\
\hline 2 & 1: 1.2 & 100 & 60 & 1 & 0 \\
\hline 3 & $1: 1.5$ & 100 & 71 & $\mathbf{0}$ & 0 \\
\hline 4 & 1: 1.8 & 100 & 70 & 1 & 0 \\
\hline 5 & 1.2: 1 & 100 & 39 & 2 & 0 \\
\hline 6 & 1.4: 1 & 100 & 41 & 2 & 0 \\
\hline 7 & 1.6: 1 & 100 & 35 & 3 & 1 \\
\hline 8 & 1.8: 1 & 90 & 24 & 4 & 3 \\
\hline
\end{tabular}

${ }^{a}$ Reaction conditions (unless otherwise specified): reactions run on a $0.2 \mathrm{mmol}$ scale, DMA ( $\left.2 \mathrm{~mL}\right)$. ${ }^{b}$ Determined by ${ }^{19} \mathrm{~F}$ NMR using fluorobenzene as an internal standard. 
Table S9. Screening of the loading amount of zinc powder ${ }^{a}$

\begin{tabular}{|c|c|c|c|c|c|}
\hline & $2 a$ & $\begin{array}{c}\mathrm{NiBr}_{2}(10 \mathrm{~mol} \%) \\
\text { 4'- }-\mathrm{ditBu}-\text { bpy }(10 \mathrm{~mol} \%)\end{array}$ & + & $\mathrm{H}_{2} \mathrm{C}=$ & \\
\hline \multirow{2}{*}{ Entry } & \multirow{2}{*}{$\mathrm{x}$} & \multirow{2}{*}{ 1, Conversion $(\%)$} & \multicolumn{3}{|c|}{ Yield $(\%)^{b}$} \\
\hline & & & 3 & 4 & 5 \\
\hline 1 & 1.8 & 100 & 70 & trace & 0 \\
\hline 2 & 2.2 & 100 & 73 & $\mathbf{0}$ & $\mathbf{0}$ \\
\hline 3 & 2.6 & 100 & 70 & 0 & 0 \\
\hline 4 & 3.0 & 100 & 71 & 0 & 0 \\
\hline 5 & 3.4 & 100 & 51 & 2 & 0 \\
\hline
\end{tabular}

${ }^{a}$ Reaction conditions (unless otherwise specified): 1 ( 0.2 mmol, 1.0 equiv), 2a (1.5 equiv), DMA (2 $\mathrm{mL}) .{ }^{b}$ Determined by ${ }^{19} \mathrm{~F}$ NMR using fluorobenzene as an internal standard.

Table S10. Control experiments ${ }^{a}$

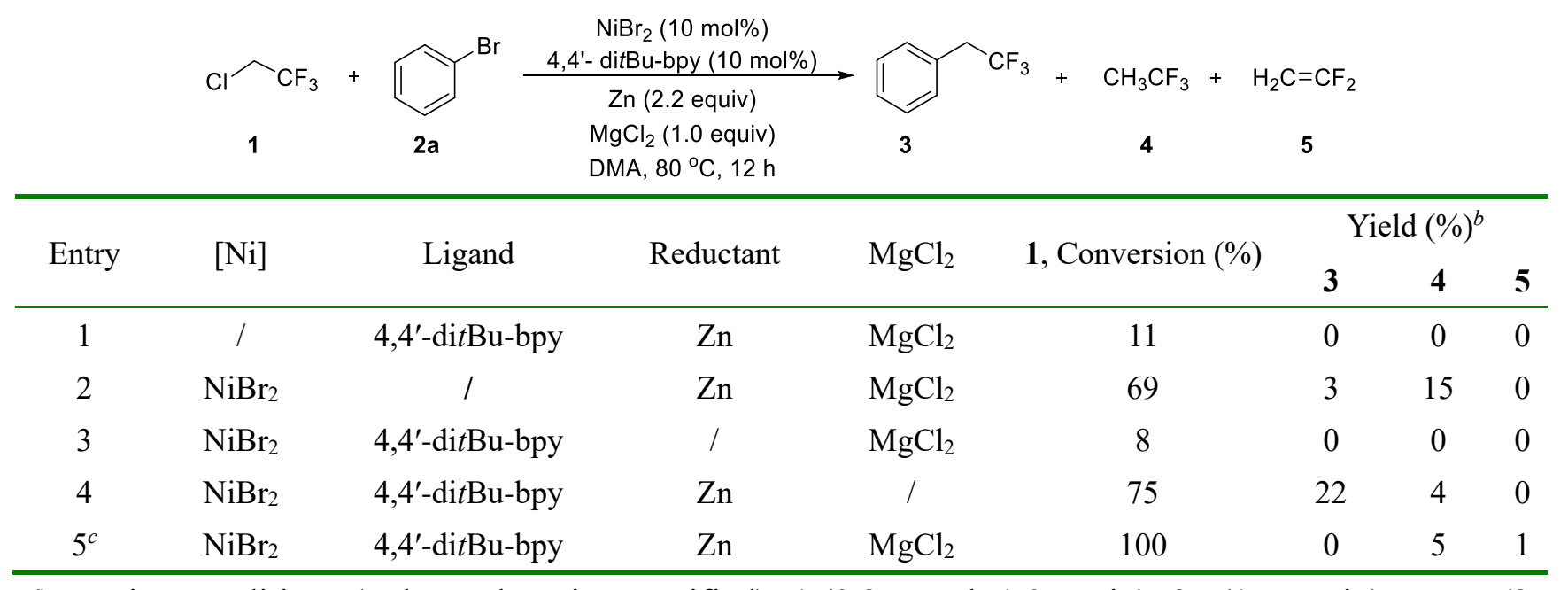

${ }^{a}$ Reaction conditions (unless otherwise specified): $\mathbf{1}$ ( $0.2 \mathrm{mmol}, 1.0$ equiv), $\mathbf{2 a}$ ( 1.5 equiv), DMA (2 $\mathrm{mL}) .{ }^{b}$ Determined by ${ }^{19} \mathrm{~F}$ NMR using fluorobenzene as an internal standard. ${ }^{c}$ Reaction ran in the absence of $\mathbf{2 a}$. 
Table S11. Optimization of the nickel-catalyzed cross-coupling of $\mathrm{CF}_{3} \mathrm{CH}_{2} \mathrm{Cl} 1$ with chlorobenzene $6 a^{a}$

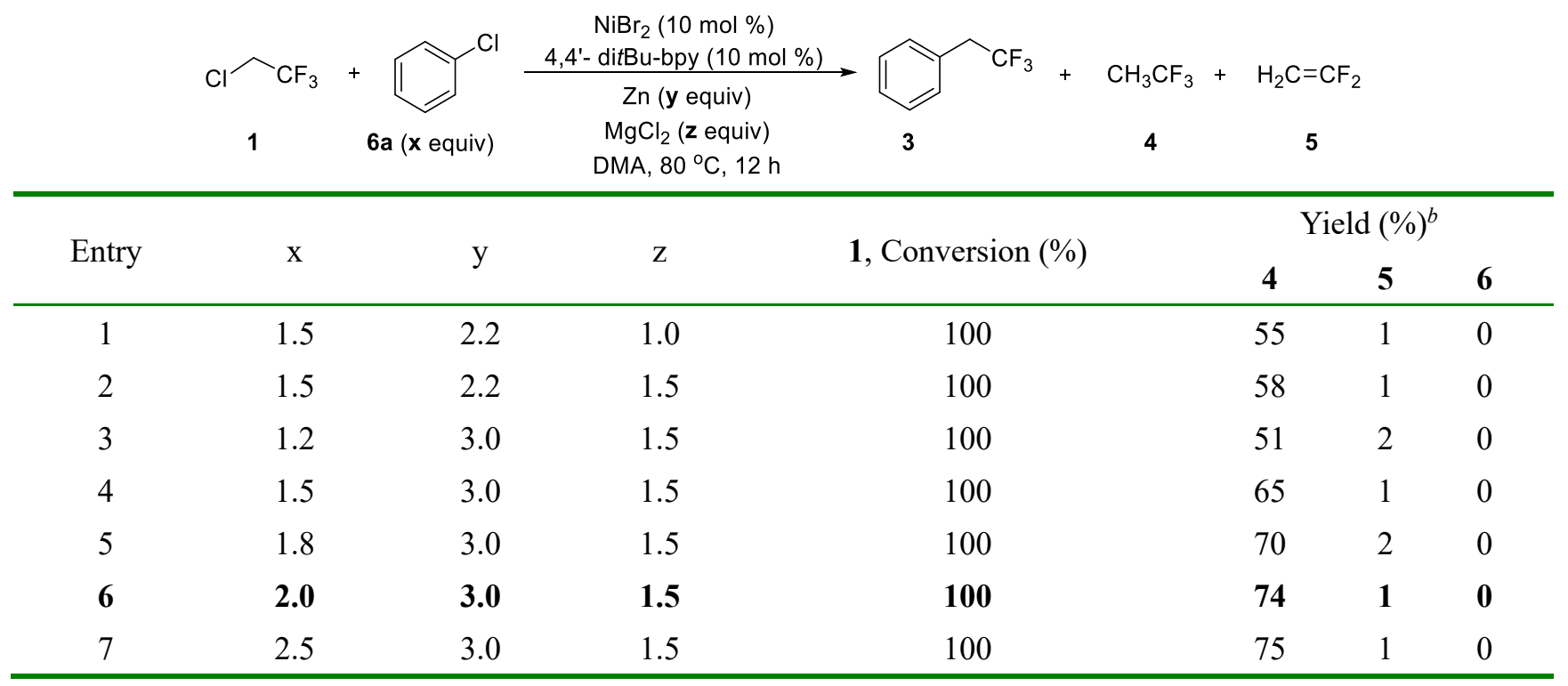

${ }^{a}$ Reaction conditions (unless otherwise specified): 1 a $(0.2 \mathrm{mmol}, 1.0$ equiv), 6a (m equiv), DMA ( 2 $\mathrm{mL}) .{ }^{b}$ Determined by ${ }^{19} \mathrm{~F}$ NMR using fluorobenzene as an internal standard.

\section{General Procedure for the Nickel-Catalyzed Cross-Coupling of $\mathrm{CF}_{3} \mathrm{CH}_{2} \mathrm{Cl}$ with (Hetero)aryl Bromides and Chlorides.}

\section{General procedure using (hetero)aryl bromides as the coupling partner:}

To a $25 \mathrm{~mL}$ of Schlenk tube were added (hetero)aryl bromide (0.6 mmol 1.5 equiv), $\mathrm{NiBr}_{2}(0.04$ mmol, $8.8 \mathrm{mg}, 0.1$ equiv), 4,4'-dtbbpy (0.04 mmol, $10.8 \mathrm{mg}, 0.1$ equiv), $\mathrm{MgCl}_{2}$ (0.4 mmol, $38.0 \mathrm{mg}$, 1.0 equiv), and zinc ( $0.88 \mathrm{mmol}, 57.2 \mathrm{mg}, 2.2$ equiv). The tube was then evacuated and refilled with argon for three times. Anhydrous DMA (4 mL) was added, followed by addition of anhydrous solution of $\mathrm{CF}_{3} \mathrm{CH}_{2} \mathrm{Cl} 1$ in DMA (1.0 equiv, $\left.0.4 \mathrm{mmol}, 2 \mathrm{M}\right)$ under Ar. The reaction mixture was stirred at $80{ }^{\circ} \mathrm{C}$ for $12 \mathrm{~h}$. The reaction was quenched by water $(4 \mathrm{~mL})$ and ethyl acetate $(4 \mathrm{~mL})$. The yield was determined by ${ }^{19} \mathrm{~F}$ NMR. Ethyl acetate $(100 \mathrm{~mL})$ was then added. The resulting reaction mixture was washed with water, brine, dried over anhydrous $\mathrm{Na}_{2} \mathrm{SO}_{4}$. After the filtration, the filtrate was concentrated and the residue was purified by silica gel column chromatography or Kugelrohr $\left(\mathrm{BUCHI}^{\circledR}\right)$ distillation. 


\section{General procedure using (hetero)aryl chlorides as the coupling partner:}

To a $25 \mathrm{~mL}$ of Schlenk tube were added (hetero)aryl chloride (0.8 mmol, 2.0 equiv), $\mathrm{NiBr}_{2}(0.04$ mmol, $8.8 \mathrm{mg}, 0.1$ equiv), 4,4'-dtbbpy (0.04 mmol, $10.8 \mathrm{mg}, 0.1$ equiv), $\mathrm{MgCl}_{2}$ (0.6 mmol, $57.2 \mathrm{mg}$, 1.5 equiv), and zinc (1.2 mmol, $79.6 \mathrm{mg}, 3.0$ equiv). The tube was then evacuated and refilled with argon for three times. Anhydrous DMA $(4 \mathrm{~mL})$ was added, followed by addition of anhydrous solution of $\mathrm{CF}_{3} \mathrm{CH}_{2} \mathrm{Cl} 1$ in DMA (1.0 equiv, $\left.0.4 \mathrm{mmol}, 2 \mathrm{M}\right)$ under Ar. The reaction mixture was stirred at $80{ }^{\circ} \mathrm{C}$ for $12 \mathrm{~h}$. The reaction was quenched by water $(4 \mathrm{~mL})$ and ethyl acetate $(4 \mathrm{~mL})$. The yield was determined by ${ }^{19} \mathrm{~F}$ NMR. Ethyl acetate $(100 \mathrm{~mL})$ was then added. The resulting reaction mixture was washed with water, brine, dried over anhydrous $\mathrm{Na}_{2} \mathrm{SO}_{4}$. After the filtration, the filtrate was concentrated and the residue was purified by silica gel column chromatography or Kugelrohr $\left(\mathrm{BUCHI}^{\circledR}\right)$ distillation.

\section{Characterization Data of Compounds 3-35e.}

$(\mathbf{2}, \mathbf{2}, 2$-Trifluoroethyl)benzene (3). The reaction was conducted on $0.2 \mathrm{mmol}$ scale. The yield of compound 3 (Ar-X: $\mathrm{X}=\mathrm{Br}, 73 \%$ yield; $\mathrm{X}=\mathrm{Cl}, 74 \%$ yield) as a light yellow oil was determined by ${ }^{19} \mathrm{~F}$ NMR. Due to the low boiling point of compound $\mathbf{3}$, it is difficult to isolate it, only NMR yield of 3 was provided. This compound is known. ${ }^{2}{ }^{19} \mathrm{~F}$ NMR $(376 \mathrm{MHz}, \delta$ $-66.3(\mathrm{t}, J=10.9 \mathrm{~Hz}, 3 \mathrm{~F})$ and GC-MS analysis confirmed its structure. GC-MS: (EI) m/z: 160 [M] $]^{+}$ $91(100)$.

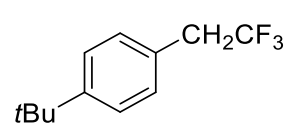

1-(tert-Butyl)-4-(2, 2, 2-trifluoroethyl)benzene (7). The reaction was conducted on $0.2 \mathrm{mmol}$ scale. The product 7 (Ar-X: $\mathrm{X}=\mathrm{Br}, 36 \mathrm{mg}, 83 \%$ yield; $\mathrm{X}=\mathrm{Cl}, 32$ mg, 74\% yield) as a colorless oil was purified by Kugelrohr distillation. This compound is known. ${ }^{3}$ H NMR (400 MHz, $\left.\mathrm{CDCl}_{3}\right): \delta 7.38(\mathrm{~d}, J=8.2 \mathrm{~Hz}, 2 \mathrm{H}), 7.24(\mathrm{~d}, J=8.2 \mathrm{~Hz}, 2 \mathrm{H}), 3.34(\mathrm{q}, J=11.0 \mathrm{~Hz}$, 2H), $1.32(\mathrm{~s}, 9 \mathrm{H}) .{ }^{13} \mathrm{C} \mathrm{NMR}\left(126 \mathrm{MHz}, \mathrm{CDCl}_{3}\right): \delta 151.0,129.8,128.0,125.9\left(\mathrm{C}-\mathrm{F},{ }^{1} J_{\mathrm{C}-\mathrm{F}}=276.6 \mathrm{~Hz}\right)$, 125.6, $39.7\left(\mathrm{C}-\mathrm{F},{ }^{2} J_{\mathrm{C}-\mathrm{F}}=29.6 \mathrm{~Hz}\right), 34.5,31.3 .{ }^{19} \mathrm{~F}$ NMR $\left(376 \mathrm{MHz}, \mathrm{CDCl}_{3}\right): \delta-66.0(\mathrm{t}, J=11.0 \mathrm{~Hz}$, 3F). MS (EI) m/z: $216[\mathrm{M}]^{+}, 216$ (100). HRMS (EI-TOF) m/z: [M] ${ }^{+}$Calcd. for $\mathrm{C}_{12} \mathrm{H}_{15} \mathrm{~F}_{3} 216.1126$; Found 216.1120. 
$\mathrm{CH}_{2} \mathrm{CF}_{3}$

1-(tert-Butyl)-3-(2, 2, 2-trifluoroethyl)benzene (8). The reaction was conducted on $0.2 \mathrm{mmol}$ scale. The product $\mathbf{8}$ (Ar-X: $\mathrm{X}=\mathrm{Br}, 39 \mathrm{mg}, 91 \%$ yield) as a colorless oil was purified by Kugelrohr distillation. ${ }^{1} \mathrm{H}$ NMR $\left(400 \mathrm{MHz}, \mathrm{CDCl}_{3}\right): \delta$ 7.45-7.36 $(\mathrm{m}, 1 \mathrm{H})$, 7.36-7.27 (m, 2H), $7.14(\mathrm{~d}, J=7.6 \mathrm{~Hz}, 1 \mathrm{H}), 3.38(\mathrm{q}, J=10.9 \mathrm{~Hz}, 2 \mathrm{H}), 1.34(\mathrm{~s}, 9 \mathrm{H}) .{ }^{13} \mathrm{C}$ NMR $(126$ $\left.\mathrm{MHz}_{\mathrm{CDCl}}\right): \delta 151.6,129.8\left(\mathrm{C}-\mathrm{F},{ }^{3} J_{\mathrm{C}-\mathrm{F}}=2.9 \mathrm{~Hz}\right), 128.3,127.23,127.18,125.9\left(\mathrm{C}-\mathrm{F},{ }^{1} J_{\mathrm{C}-\mathrm{F}}=276.8\right.$ $\mathrm{Hz}), 125.0,40.5\left(\mathrm{C}-\mathrm{F},{ }^{2} J_{\mathrm{C}-\mathrm{F}}=29.5 \mathrm{~Hz}\right), 34.6,31.3 .{ }^{19} \mathrm{~F}$ NMR $\left(376 \mathrm{MHz}, \mathrm{CDCl}_{3}\right): \delta-65.9(\mathrm{t}, J=10.9$ $\mathrm{Hz}, 3 \mathrm{~F}$ ). MS (EI) m/z: $216[\mathrm{M}]^{+}, 216$ (100). HRMS (EI-TOF) m/z: [M] ${ }^{+}$Calcd. for $\mathrm{C}_{12} \mathrm{H}_{15} \mathrm{~F}_{3}$ 216.1126; Found 216.1129.

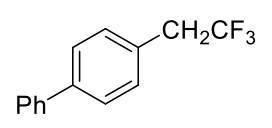

4-(2, 2, 2-Trifluoroethyl)-1, 1'-biphenyl (9). The product 9 (Ar-X: X = Br, 59 mg, $62 \%$ yield; $\mathrm{X}=\mathrm{Cl}, 67 \mathrm{mg}, 71 \%$ yield) as a white solid (m.p. $72-73{ }^{\circ} \mathrm{C}$ ) was purified by silica gel column chromatography (petroleum ether). This compound is known. ${ }^{4} \mathrm{H}$ NMR (400 $\left.\mathrm{MHz}, \mathrm{CDCl}_{3}\right): \delta$ 7.66-7.56 (m, 4H), 7.52-7.44 (m, 2H), 7.42-7.36 (m, 3H), $3.43(\mathrm{q}, J=10.8 \mathrm{~Hz}, 2 \mathrm{H})$. ${ }^{13} \mathrm{C} \mathrm{NMR}\left(126 \mathrm{MHz}, \mathrm{CDCl}_{3}\right): \delta 141.1,140.5,130.5,129.1\left(\mathrm{C}-\mathrm{F},{ }^{3} J_{\mathrm{C}-\mathrm{F}}=2.9 \mathrm{~Hz}\right), 128.8,127.5,127.4$, 127.1, $125.8\left(\mathrm{C}-\mathrm{F},{ }^{1} J_{\mathrm{C}-\mathrm{F}}=276.8 \mathrm{~Hz}\right), 39.9\left(\mathrm{C}-\mathrm{F},{ }^{2} J_{\mathrm{C}-\mathrm{F}}=29.7 \mathrm{~Hz}\right) .{ }^{19} \mathrm{~F}$ NMR $\left(376 \mathrm{MHz}, \mathrm{CDCl}_{3}\right): \delta$ $-65.9(\mathrm{t}, J=10.8 \mathrm{~Hz}, 3 \mathrm{~F}) . \mathrm{MS}(\mathrm{EI}) \mathrm{m} / \mathrm{z}: 236[\mathrm{M}]^{+}, 167$ (100). HRMS (EI-TOF) m/z: [M] $]^{+}$Calcd. for $\mathrm{C}_{14} \mathrm{H}_{11} \mathrm{~F}_{3} 236.0813$; Found 236.0817.

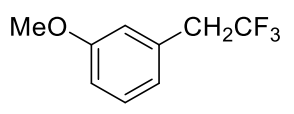

1-Methoxy-3-(2, 2, 2-trifluoroethyl)benzene (10). The reaction was conducted on $0.2 \mathrm{mmol}$ scale. The product $\mathbf{1 0}$ (Ar-X: $\mathrm{X}=\mathrm{Br}, 31 \mathrm{mg}, 82 \%$ yield; $\mathrm{X}=\mathrm{Cl}, 24$ $\mathrm{mg}, 62 \%$ yield) as a colorless oil was distilled by kugelrohr $\left(\mathrm{BUCHI}^{\circledR}\right)$. This compound is known. ${ }^{5}$ ${ }^{1} \mathrm{H}$ NMR (400 MHz, $\left.\mathrm{CDCl}_{3}\right): \delta 7.29(\mathrm{t}, J=7.8 \mathrm{~Hz}, 1 \mathrm{H}), 6.94-6.88(\mathrm{~m}, 2 \mathrm{H}), 6.86(\mathrm{~s}, 1 \mathrm{H}), 3.83(\mathrm{~s}, 3 \mathrm{H})$, $3.36(\mathrm{q}, J=10.8 \mathrm{~Hz}, 2 \mathrm{H}) .{ }^{13} \mathrm{C} \mathrm{NMR}\left(126 \mathrm{MHz}, \mathrm{CDCl}_{3}\right): \delta 159.7,131.5\left(\mathrm{C}-\mathrm{F},{ }^{3} J_{\mathrm{C}-\mathrm{F}}=2.9 \mathrm{~Hz}\right), 129.6$, $125.8\left(\mathrm{C}-\mathrm{F},{ }^{1} J_{\mathrm{C}-\mathrm{F}}=276.7 \mathrm{~Hz}\right), 122.4,115.9,113.4,55.2,40.2\left(\mathrm{C}-\mathrm{F},{ }^{2} J_{\mathrm{C}-\mathrm{F}}=29.7 \mathrm{~Hz}\right) .{ }^{19} \mathrm{~F}$ NMR $(376 \mathrm{MHz}$, $\left.\mathrm{CDCl}_{3}\right): \delta-65.8(\mathrm{t}, J=10.8 \mathrm{~Hz}, 3 \mathrm{~F}) . \mathrm{MS}(\mathrm{EI}) \mathrm{m} / \mathrm{z}: 190[\mathrm{M}]^{+}, 190(100) . \mathrm{HRMS}(\mathrm{EI}-\mathrm{TOF}) \mathrm{m} / \mathrm{z}:[\mathrm{M}]^{+}$ Calcd. for $\mathrm{C}_{9} \mathrm{H}_{9} \mathrm{~F}_{3} \mathrm{O} 190.0605$; Found 190.0603. 
$\mathrm{BnO}$<smiles>F[C+](F)Cc1ccccc1</smiles>

1-(Benzyloxy)-3-(2, 2, 2-trifluoroethyl)benzene (11). The product 11 (ArX: $\mathrm{X}=$

$\mathrm{Br}, 75 \mathrm{mg}, 71 \%$ yield) as a yellow oil was purified by silica gel column chromatography (PE:EA $=30: 1)$. This compound is known. ${ }^{6}{ }^{1} \mathrm{H}$ NMR $\left(400 \mathrm{MHz}, \mathrm{CDCl}_{3}\right)$ : $\delta$ 7.48-7.38 (m, 4H), 7.38-7.32 (m, 1H), 7.29 (t, $J=7.6 \mathrm{~Hz}, 1 \mathrm{H}), 6.99-6.89(\mathrm{~m}, 3 \mathrm{H}), 5.08(\mathrm{~s}, 2 \mathrm{H}), 3.35$ $(\mathrm{q}, J=10.8 \mathrm{~Hz}, 2 \mathrm{H}) .{ }^{13} \mathrm{C} \mathrm{NMR}\left(126 \mathrm{MHz}, \mathrm{CDCl}_{3}\right): \delta 158.9,136.7,131.6\left(\mathrm{C}-\mathrm{F},{ }^{3} J_{\mathrm{C}-\mathrm{F}}=2.8 \mathrm{~Hz}\right), 129.7$, 128.6, 128.0, 127.5, $125.7\left(\mathrm{C}-\mathrm{F},{ }^{1} J_{\mathrm{C}-\mathrm{F}}=276.8 \mathrm{~Hz}\right), 122.7,116.9,114.3,70.0,40.2\left(\mathrm{C}-\mathrm{F},{ }^{2} J_{\mathrm{C}-\mathrm{F}}=29.7\right.$ Hz). ${ }^{19} \mathrm{~F}$ NMR $\left(376 \mathrm{MHz}, \mathrm{CDCl}_{3}\right): \delta-65.8(\mathrm{t}, J=10.8 \mathrm{~Hz}, 3 \mathrm{~F}) . \mathrm{MS}(\mathrm{EI}) \mathrm{m} / \mathrm{z}: 266[\mathrm{M}]^{+}, 91(100)$. HRMS (EI-TOF) m/z: [M] Calcd. for $\mathrm{C}_{15} \mathrm{H}_{13} \mathrm{~F}_{3} \mathrm{O} 266.0918$; Found 266.0922.

$\overbrace{}^{\mathrm{N}} \mathrm{CH}_{2} \mathrm{CF}_{3}$ 4-(3-(2, 2, 2-Trifluoroethyl)phenyl)morpholine (12). The product 12 (ArX: $\mathrm{X}=\mathrm{Br}, 40 \mathrm{mg}, 41 \%$ yield) as a yellow solid (m.p. 59-61 ${ }^{\circ} \mathrm{C}$ ) was purified by silica gel column chromatography (PE:EA $=5: 1) .{ }^{1} \mathrm{H}$ NMR $\left(400 \mathrm{MHz}, \mathrm{CDCl}_{3}\right): \delta 7.27(\mathrm{t}, J=7.9 \mathrm{~Hz}$, $1 \mathrm{H}), 6.90(\mathrm{dd}, J=7.6 \mathrm{~Hz}, 1.9 \mathrm{~Hz}, 1 \mathrm{H}), 6.82(\mathrm{~d}, J=7.5 \mathrm{~Hz}, 2 \mathrm{H}), 3.88-3.86(\mathrm{~m}, 4 \mathrm{H}), 3.34(\mathrm{q}, J=$ $10.8 \mathrm{~Hz}, 2 \mathrm{H}), 3.19-3.16(\mathrm{~m}, 4 \mathrm{H}) .{ }^{13} \mathrm{C} \mathrm{NMR}\left(126 \mathrm{MHz}, \mathrm{CDCl}_{3}\right): \delta 151.5,131.1\left(\mathrm{C}-\mathrm{F},{ }^{3} J_{\mathrm{C}-\mathrm{F}}=2.9 \mathrm{~Hz}\right)$, $129.4,125.8\left(\mathrm{C}-\mathrm{F},{ }^{1} J_{\mathrm{C}-\mathrm{F}}=276.8 \mathrm{~Hz}\right), 121.6,117.4,115.2,66.9,49.1,40.4\left(\mathrm{C}-\mathrm{F},{ }^{2} J_{\mathrm{C}-\mathrm{F}}=29.5 \mathrm{~Hz}\right) .{ }^{19} \mathrm{~F}$ NMR (376 MHz, $\left.\mathrm{CDCl}_{3}\right): \delta-65.7(\mathrm{t}, J=10.9 \mathrm{~Hz}, 3 \mathrm{~F}) . \mathrm{MS}(\mathrm{EI}) \mathrm{m} / \mathrm{z}: 245[\mathrm{M}]^{+}, 187$ (100). HRMS (EI-TOF) m/z: [M] ${ }^{+}$Calcd. for $\mathrm{C}_{12} \mathrm{H}_{14} \mathrm{~F}_{3} \mathrm{NO} 245.1027$; Found 245.1022.

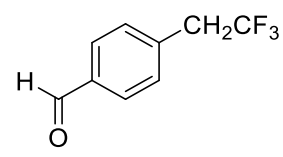

4-(2, 2, 2-Trifluoroethyl)benzaldehyde (13). The reaction was conducted on 0.2 mmol scale. The product 13 (ArX: $\mathrm{X}=\mathrm{Br}, 49 \mathrm{mg}, 65 \%$ yield) as a white solid was purified by silica gel column chromatography $(\mathrm{PE}: \mathrm{EA}=40: 1)$. This compound is known. ${ }^{3} \mathrm{H}_{\mathrm{NMR}}\left(400 \mathrm{MHz}, \mathrm{CDCl}_{3}\right)$ : $\delta 10.02(\mathrm{~s}, 1 \mathrm{H}), 7.87(\mathrm{~d}, J=8.2 \mathrm{~Hz}, 2 \mathrm{H}), 7.47$ (d, $J=8.0 \mathrm{~Hz}, 2 \mathrm{H}), 3.46(\mathrm{q}, J=10.6 \mathrm{~Hz}, 2 \mathrm{H}) .{ }^{13} \mathrm{C} \mathrm{NMR}\left(126 \mathrm{MHz}, \mathrm{CDCl}_{3}\right): \delta 191.6,136.7\left(\mathrm{C}-\mathrm{F},{ }^{3} J_{\mathrm{C}-\mathrm{F}}=\right.$ $2.9 \mathrm{~Hz}), 130.9,130.0,125.3\left(\mathrm{C}-\mathrm{F},{ }^{1} J_{\mathrm{C}-\mathrm{F}}=277.0 \mathrm{~Hz}\right), 40.3\left(\mathrm{C}-\mathrm{F},{ }^{2} J_{\mathrm{C}-\mathrm{F}}=30.1 \mathrm{~Hz}\right) .{ }^{19} \mathrm{~F}$ NMR $(376 \mathrm{MHz}$, $\left.\mathrm{CDCl}_{3}\right): \delta-65.5(\mathrm{t}, J=10.6 \mathrm{~Hz}, 3 \mathrm{~F}) . \mathrm{MS}(\mathrm{EI}) \mathrm{m} / \mathrm{z}: 188[\mathrm{M}]^{+}, 204$ (100). HRMS (EI-TOF) m/z: [M] ${ }^{+}$ Calcd. for $\mathrm{C}_{9} \mathrm{H}_{7} \mathrm{~F}_{3} \mathrm{O}$ 188.0449; Found 188.0444. 
$\mathrm{OHC}$ 3-(2, 2, 2-Trifluoroethyl)benzaldehyde (14). The reaction was conducted on 0.2 mmol scale. The product 14 ( $\mathrm{ArX}: \mathrm{X}=\mathrm{Br}, 49 \mathrm{mg}, 65 \%$ yield) as a colorless oil was purified by silica gel column chromatography (PE:EA $=40: 1$ ). This compound is known. ${ }^{1}{ }^{1} \mathrm{H}$ NMR (500 MHz, $\left.\mathrm{CDCl}_{3}\right): \delta 10.01$ (s, 1H), $7.86(\mathrm{dd}, J=7.2 \mathrm{~Hz}, 1.6 \mathrm{~Hz}, 1 \mathrm{H}), 7.82(\mathrm{~s}, 1 \mathrm{H}), 7.58-7.52$ (m, 2H), 3.46 (q, $J=10.7 \mathrm{~Hz}, 2 \mathrm{H}) .{ }^{13} \mathrm{C}$ NMR (126 MHz, $\mathrm{CDCl}_{3}$ ): $\delta$ 191.7, 136.8, 136.0, 131.3 (C-F, $\left.{ }^{3} J_{\mathrm{C}-\mathrm{F}}=3.0 \mathrm{~Hz}\right), 131.0,129.6,129.4,125.4\left(\mathrm{C}-\mathrm{F},{ }^{1} J_{\mathrm{C}-\mathrm{F}}=276.8 \mathrm{~Hz}\right), 39.9\left(\mathrm{C}-\mathrm{F},{ }^{2} J_{\mathrm{C}-\mathrm{F}}=30.0 \mathrm{~Hz}\right) .{ }^{19} \mathrm{~F}$ NMR (376 MHz, $\left.\mathrm{CDCl}_{3}\right): \delta-65.9(\mathrm{t}, J=10.7 \mathrm{~Hz}, 3 \mathrm{~F})$. MS (EI) m/z: $188[\mathrm{M}]^{+}, 187$ (100). HRMS (EI-TOF) m/z: [M] $]^{+}$Calcd. for $\mathrm{C}_{9} \mathrm{H}_{7} \mathrm{~F}_{3} \mathrm{O}$ 188.0449; Found 188.0450 .<smiles>CC(=O)c1cccc(CC[AsH3+])c1</smiles>

1-(3-(2, 2, 2-Trifluoroethyl)phenyl)ethan-1-one (15). The reaction was conducted on $0.2 \mathrm{mmol}$ scale. The product 15 (ArX: $\mathrm{X}=\mathrm{Br}, 29 \mathrm{mg}, 71 \%$ yield; $\mathrm{X}=$ $\mathrm{Cl}, 28 \mathrm{mg}, 70 \%$ yield) as a yellow oil was purified by silica gel column chromatography (PE:EA = 30:1). This compound is known. ${ }^{3}{ }^{1} \mathrm{H}$ NMR (400 MHz, $\left.\mathrm{CDCl}_{3}\right): \delta 7.92(\mathrm{~d}, J=7.4 \mathrm{~Hz}, 1 \mathrm{H}), 7.89$ (s, 1H), 7.52-7.43 (m, 2H), 3.43 (q, $J=10.7 \mathrm{~Hz}, 2 \mathrm{H}), 2.60(\mathrm{~s}, 3 \mathrm{H}) .{ }^{13} \mathrm{C}$ NMR $\left(126 \mathrm{MHz}, \mathrm{CDCl}_{3}\right): \delta$ 197.5, 137.5, 134.6, $130.7\left(\mathrm{C}-\mathrm{F},{ }^{3} J_{\mathrm{C}-\mathrm{F}}=2.9 \mathrm{~Hz}\right), 129.9,129.0,128.1,125.5\left(\mathrm{C}-\mathrm{F},{ }^{1} J_{\mathrm{C}-\mathrm{F}}=276.8 \mathrm{~Hz}\right)$, $40.0\left(\mathrm{C}-\mathrm{F},{ }^{2} J_{\mathrm{C}-\mathrm{F}}=30.0 \mathrm{~Hz}\right), 26.6 .{ }^{19} \mathrm{~F}$ NMR $\left(376 \mathrm{MHz}, \mathrm{CDCl}_{3}\right): \delta-65.9(\mathrm{t}, J=10.7 \mathrm{~Hz}, 3 \mathrm{~F})$. MS (EI) m/z: $202[\mathrm{M}]^{+}, 187$ (100). HRMS (EI-TOF) m/z: [M] Calcd. for $\mathrm{C}_{10} \mathrm{H}_{9} \mathrm{~F}_{3} \mathrm{O}$ 202.0605; Found 202.0609 .

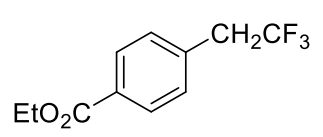

Ethyl 4-(2, 2, 2-trifluoroethyl)benzoate (16). The product 16 (ArX: $\mathrm{X}=\mathrm{Br}, 51$ $\mathrm{mg}, 55 \%$ yield; $\mathrm{X}=\mathrm{Cl}, 70 \mathrm{mg}, 75 \%$ yield) as a colorless oil was purified by silica gel column chromatography (PE:EA $=20: 1)$. This compound is known. ${ }^{1} \mathrm{H}$ NMR $(400 \mathrm{MHz}$, $\left.\mathrm{CDCl}_{3}\right): \delta 8.04$ (d, $\left.J=8.2 \mathrm{~Hz}, 2 \mathrm{H}\right), 7.37(\mathrm{~d}, J=8.1 \mathrm{~Hz}, 2 \mathrm{H}), 4.38$ (q, $\left.J=7.2 \mathrm{~Hz}, 2 \mathrm{H}\right), 3.43$ (q, $J=$ $10.7 \mathrm{~Hz}, 2 \mathrm{H}), 1.39(\mathrm{t}, J=7.2 \mathrm{~Hz}, 3 \mathrm{H}) .{ }^{13} \mathrm{C}$ NMR $\left(126 \mathrm{MHz}, \mathrm{CDCl}_{3}\right): \delta 166.1,134.9\left(\mathrm{C}-\mathrm{F},{ }^{3} J_{\mathrm{C}-\mathrm{F}}=2.9\right.$ $\mathrm{Hz}), 130.4,130.1,129.8,125.4\left(\mathrm{C}-\mathrm{F},{ }^{1} J_{\mathrm{C}-\mathrm{F}}=275.9 \mathrm{~Hz}\right), 61.1,40.1\left(\mathrm{C}-\mathrm{F},{ }^{2} J_{\mathrm{C}-\mathrm{F}}=30.0 \mathrm{~Hz}\right), 14.3 .{ }^{19} \mathrm{~F}$ NMR (376 MHz, CDCl $\left.)_{3}\right): \delta-65.7(\mathrm{t}, J=10.7 \mathrm{~Hz}, 3 \mathrm{~F})$. MS (EI) m/z: $232[\mathrm{M}]^{+}, 187$ (100). HRMS (EI-TOF) m/z: [M] Calcd. for $\mathrm{C}_{11} \mathrm{H}_{11} \mathrm{~F}_{3} \mathrm{O}_{2} 232.0711$; Found 232.0705. 
$\mathrm{EtO}_{2} \mathrm{C} \mathrm{CH}_{2} \mathrm{CF}_{3}$

Ethyl 3-(2, 2, 2-trifluoroethyl)benzoate (17). The product 17 (ArX: X = Br, 51 $\mathrm{mg}, 55 \%$ yield, reaction run for $15 \mathrm{~h} ; \mathrm{X}=\mathrm{Cl}, 63 \mathrm{mg}, 68 \%$ yield) was a colorless oil purified by silica gel column chromatography $(\mathrm{PE}: \mathrm{EA}=20: 1)$. This compound is known. ${ }^{3} \mathrm{H}$ $\operatorname{NMR}\left(400 \mathrm{MHz}, \mathrm{CDCl}_{3}\right): \delta 8.02(\mathrm{~d}, J=7.6 \mathrm{~Hz}, 1 \mathrm{H}), 7.99(\mathrm{~s}, 1 \mathrm{H}), 7.49(\mathrm{~d}, J=7.6 \mathrm{~Hz}, 1 \mathrm{H}), 7.44(\mathrm{t}, J$ $=7.6 \mathrm{~Hz}, 1 \mathrm{H}), 4.39(\mathrm{q}, J=7.1 \mathrm{~Hz}, 2 \mathrm{H}), 3.42(\mathrm{q}, J=0.7 \mathrm{~Hz}, 2 \mathrm{H}), 1.40(\mathrm{t}, J=7.1 \mathrm{~Hz}, 3 \mathrm{H}) .{ }^{13} \mathrm{C} \mathrm{NMR}$ $\left(126 \mathrm{MHz}, \mathrm{CDCl}_{3}\right): \delta 166.1,134.4,131.2,131.0,130.4\left(\mathrm{C}-\mathrm{F},{ }^{3} J_{\mathrm{C}-\mathrm{F}}=3.0 \mathrm{~Hz}\right), 129.3,128.7,125.5$ $\left(\mathrm{C}-\mathrm{F},{ }^{1} J_{\mathrm{C}-\mathrm{F}}=276.9 \mathrm{~Hz}\right), 61.1,40.0\left(\mathrm{C}-\mathrm{F},{ }^{2} J_{\mathrm{C}-\mathrm{F}}=30.2 \mathrm{~Hz}\right), 14.3 .{ }^{19} \mathrm{~F}$ NMR $\left(376 \mathrm{MHz}, \mathrm{CDCl}_{3}\right): \delta-66.0$ (t, $J=10.7 \mathrm{~Hz}, 3 \mathrm{~F}) . \mathrm{MS}(\mathrm{EI}) \mathrm{m} / \mathrm{z}: 232[\mathrm{M}]^{+}, 187$ (100). HRMS (EI-TOF) m/z: [M] Calcd. for $\mathrm{C}_{11} \mathrm{H}_{11} \mathrm{~F}_{3} \mathrm{O}_{2} 232.0711$; Found 232.0714.

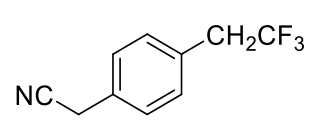

2-(4-(2, 2, 2-Trifluoroethyl)phenyl)acetonitrile (18). The product 18 (ArX: X= Br, $65 \mathrm{mg}, 82 \%$ yield; $\mathrm{X}=\mathrm{Cl}, 49 \mathrm{mg}, 61 \%$ yield) as a white solid (m.p. $79-80{ }^{\circ} \mathrm{C}$ ) was purified by silica gel column chromatography $(\mathrm{PE}: \mathrm{EA}=8: 1)$. This compound is known. ${ }^{1} \mathrm{H}$ NMR (400 MHz, $\left.\mathrm{CDCl}_{3}\right): \delta$ 7.35-7.33 (m, 4H), $3.76(\mathrm{~s}, 2 \mathrm{H}), 3.38(\mathrm{q}, J=10.7 \mathrm{~Hz}, 2 \mathrm{H}) .{ }^{13} \mathrm{C}$ NMR $\left(126 \mathrm{MHz}, \mathrm{CDCl}_{3}\right): \delta 130.9,130.1\left(\mathrm{C}-\mathrm{F},{ }^{3} J_{\mathrm{C}-\mathrm{F}}=2.9 \mathrm{~Hz}\right), 129.9,128.2,125.5\left(\mathrm{C}-\mathrm{F},{ }^{1} J_{\mathrm{C}-\mathrm{F}}=276.8 \mathrm{~Hz}\right)$, 117.6, $39.7\left(\mathrm{C}-\mathrm{F},{ }^{2} J_{\mathrm{C}-\mathrm{F}}=29.9 \mathrm{~Hz}\right), 23.2 .{ }^{19} \mathrm{~F} \mathrm{NMR}\left(376 \mathrm{MHz}, \mathrm{CDCl}_{3}\right): \delta-66.0(\mathrm{t}, J=10.7 \mathrm{~Hz}, 3 \mathrm{~F})$. MS (EI) m/z: $199[\mathrm{M}]^{+}, 130$ (100). HRMS (EI-TOF) m/z: [M] $]^{+}$Calcd. for $\mathrm{C}_{10} \mathrm{H}_{8} \mathrm{~F}_{3} \mathrm{~N}$ 199.0609; Found 199.0610.

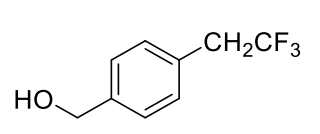

(4-(2, 2, 2-Trifluoroethyl)phenyl)methanol (19). The product 19 (ArX: X=Br, $40 \mathrm{mg}, 52 \%$ yield; $\mathrm{X}=\mathrm{Cl}, 42 \mathrm{mg}, 55 \%$ yield) as a white solid (m.p. $63-64{ }^{\circ} \mathrm{C}$ ) was purified by silica gel column chromatography (PE:EA = 5:1). ${ }^{1} \mathrm{H}$ NMR $\left(400 \mathrm{MHz}, \mathrm{CDCl}_{3}\right): \delta$ $7.35(\mathrm{~d}, J=7.9 \mathrm{~Hz}, 2 \mathrm{H}), 7.29$ (d, $J=8.0 \mathrm{~Hz}, 2 \mathrm{H}), 4.68(\mathrm{~s}, 2 \mathrm{H}), 3.37$ (q, $J=10.8 \mathrm{~Hz}, 2 \mathrm{H}) .2 .00$ (br, 1H). ${ }^{13} \mathrm{C}$ NMR $\left(126 \mathrm{MHz} \mathrm{CDCl}_{3}\right): \delta 140.8,130.2,129.5\left(\mathrm{C}-\mathrm{F},{ }^{3} J_{\mathrm{C}-\mathrm{F}}=2.9 \mathrm{~Hz}\right), 127.2,125.7(\mathrm{C}-\mathrm{F}$, $\left.{ }^{1} J_{\mathrm{C}-\mathrm{F}}=276.7 \mathrm{~Hz}\right), 64.8,39.9\left(\mathrm{C}-\mathrm{F},{ }^{2} J_{\mathrm{C}-\mathrm{F}}=29.9 \mathrm{~Hz}\right) .{ }^{19} \mathrm{~F}$ NMR $\left(376 \mathrm{MHz}, \mathrm{CDCl}_{3}\right): \delta-66.0(\mathrm{t}, J=10.8$ $\mathrm{Hz}, 3 \mathrm{~F}$ ). MS (EI) m/z: $190[\mathrm{M}]^{+}, 107$ (100). HRMS (EI-TOF) m/z: [M] ${ }^{+}$Calcd. for $\mathrm{C}_{9} \mathrm{H}_{9} \mathrm{~F}_{3} \mathrm{O}$ 190.0605; Found 190.0601. 
Trimethyl(4-(2, 2, 2-trifluoroethyl)phenyl)silane (20). The product 20 (ArX: $\mathrm{X}=$ $\mathrm{Br}, 84 \mathrm{mg}, 90 \%$ yield) as a colorless oil was distilled by kugelrohr (BUCHI $\left.{ }^{\circledR}\right)$. This compound is known. ${ }^{2} \mathrm{H}$ NMR $\left(400 \mathrm{MHz}, \mathrm{CDCl}_{3}\right): \delta 7.58(\mathrm{~d}, J=7.9 \mathrm{~Hz}, 2 \mathrm{H}), 7.34(\mathrm{~d}, J=7.6$ $\mathrm{Hz}, 2 \mathrm{H}), 3.40$ (q, $J=10.9 \mathrm{~Hz}, 2 \mathrm{H}) .0 .33$ (s, 9H). ${ }^{13} \mathrm{C} \mathrm{NMR}\left(126 \mathrm{MHz}, \mathrm{CDCl}_{3}\right): \delta 140.4,133.7,130.6$ $\left(\mathrm{C}-\mathrm{F},{ }^{3} J_{\mathrm{C}-\mathrm{F}}=2.9 \mathrm{~Hz}\right), 129.5,125.8\left(\mathrm{C}-\mathrm{F},{ }^{1} J_{\mathrm{C}-\mathrm{F}}=275.7 \mathrm{~Hz}\right), 40.2\left(\mathrm{C}-\mathrm{F},{ }^{2} J_{\mathrm{C}-\mathrm{F}}=29.6 \mathrm{~Hz}\right),-1.2 .{ }^{19} \mathrm{~F}$ NMR (376 MHz, $\left.\mathrm{CDCl}_{3}\right): \delta-65.8(\mathrm{t}, J=10.8 \mathrm{~Hz}, 3 \mathrm{~F}) . \mathrm{MS}(\mathrm{EI}) \mathrm{m} / \mathrm{z}: 232[\mathrm{M}]^{+}, 217$ (100). HRMS (EI-TOF) $\mathrm{m} / \mathrm{z}:[\mathrm{M}]^{+}$Calcd. for $\mathrm{C}_{11} \mathrm{H}_{15} \mathrm{~F}_{3} \mathrm{Si} 232.0895$; Found 232.0887.

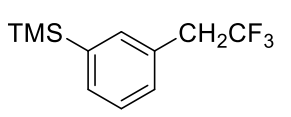

Trimethyl(3-(2, 2, 2-trifluoroethyl)phenyl)silane (21). The product 21 (ArX: $\mathrm{X}=$

$\mathrm{Br}, 86 \mathrm{mg}, 93 \%$ yield) as a colorless oil was distilled by kugelrohr $\left(\mathrm{BUCHI}^{\circledR}\right) .{ }^{1} \mathrm{H}$ NMR (400 MHz, $\left.\mathrm{CDCl}_{3}\right): \delta 7.55(\mathrm{~d}, J=7.2 \mathrm{~Hz}, 1 \mathrm{H}), 7.47$ (s, 1H), 7.39 (t, $\left.J=7.4 \mathrm{~Hz}, 2 \mathrm{H}\right), 7.33(\mathrm{~d}, J$ $=7.6 \mathrm{~Hz}, 1 \mathrm{H}), 3.41(\mathrm{~d}, J=10.9 \mathrm{~Hz}, 2 \mathrm{H}), 0.33(\mathrm{~s}, 9 \mathrm{H}) .{ }^{13} \mathrm{C} \mathrm{NMR}\left(126 \mathrm{MHz}, \mathrm{CDCl}_{3}\right): \delta 141.2,135.1$, 133.0, 130.5, $129.5\left(\mathrm{C}-\mathrm{F},{ }^{3} J_{\mathrm{C}-\mathrm{F}}=3.2 \mathrm{~Hz}\right), 128.0,125.9\left(\mathrm{C}-\mathrm{F},{ }^{1} J_{\mathrm{C}-\mathrm{F}}=275.7 \mathrm{~Hz}\right), 40.4\left(\mathrm{C}-\mathrm{F},{ }^{2} J_{\mathrm{C}-\mathrm{F}}=29.7\right.$ $\left.\mathrm{Hz}),-1.2 .{ }^{19} \mathrm{~F} \mathrm{NMR}\left(376 \mathrm{MHz}, \mathrm{CDCl}_{3}\right): \delta-65.9(\mathrm{t}, J=10.9 \mathrm{~Hz}, 3 \mathrm{~F}) . \mathrm{MS}(\mathrm{EI}) \mathrm{m} / \mathrm{z}: 232[\mathrm{M}]\right]^{+}, 217$ (100). HRMS (EI-TOF) m/z: [M] $]^{+}$Calcd. for $\mathrm{C}_{11} \mathrm{H}_{15} \mathrm{~F}_{3} \mathrm{Si} 232.0895$; Found 232.0902.

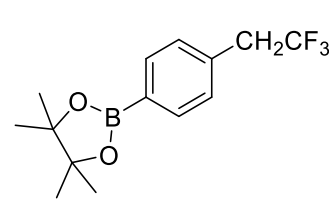

\section{4, 4, 5, 5-Tetramethyl-2-(4-(2, 2, 2-trifluoroethyl)phenyl)-1， 3,} 2-dioxaborolane (22). The product 22 ( $\mathrm{ArX}$ : $\mathrm{X}=\mathrm{Br}, 102 \mathrm{mg}, 94 \%$ yield; $\mathrm{X}=\mathrm{Cl}$, $90 \mathrm{mg}, 79 \%$ yield) as a white solid (m.p. $68-70{ }^{\circ} \mathrm{C}$ ) was purified by silica gel column chromatography $(\mathrm{PE}: \mathrm{EA}=50: 1)$. This compound is known. ${ }^{8}{ }^{1} \mathrm{H} \mathrm{NMR}\left(400 \mathrm{MHz}, \mathrm{CDCl}_{3}\right): \delta$ $7.80(\mathrm{~d}, J=7.9 \mathrm{~Hz}, 2 \mathrm{H}), 7.31(\mathrm{~d}, J=7.7 \mathrm{~Hz}, 2 \mathrm{H}), 3.38(\mathrm{q}, J=10.8 \mathrm{~Hz}, 2 \mathrm{H}) .1 .35(\mathrm{~s}, 12 \mathrm{H}) .{ }^{13} \mathrm{C} \mathrm{NMR}$ $\left(126 \mathrm{MHz} \mathrm{CDCl}_{3}\right): \delta 135.1,133.1\left(\mathrm{C}-\mathrm{F},{ }^{3} J_{\mathrm{C}-\mathrm{F}}=2.8 \mathrm{~Hz}\right), 129.5,125.7\left(\mathrm{C}-\mathrm{F},{ }^{1} J_{\mathrm{C}-\mathrm{F}}=276.9 \mathrm{~Hz}\right), 83.9$, $40.4\left(\mathrm{C}-\mathrm{F},{ }^{2} J_{\mathrm{C}-\mathrm{F}}=29.7 \mathrm{~Hz}\right), 24.9 .{ }^{19} \mathrm{~F} \mathrm{NMR}\left(376 \mathrm{MHz}, \mathrm{CDCl}_{3}\right): \delta-65.8(\mathrm{t}, J=10.8 \mathrm{~Hz}, 3 \mathrm{~F}) . \mathrm{MS}(\mathrm{EI})$ m/z: $286[\mathrm{M}]^{+}, 187$ (100). HRMS (EI-TOF) m/z: [M] $]^{+}$Calcd. for $\mathrm{C}_{14} \mathrm{H}_{17} \mathrm{~F}_{3} \mathrm{O}_{2} \mathrm{~B}^{10}$ 284.1310; Found 284.1299. 


\section{Gram-scale synthesis of compound 22.}

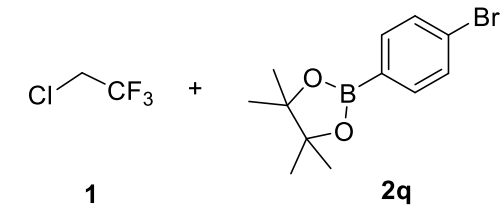

$(7.0 \mathrm{mmol}, 1.0 \mathrm{eq}) \quad(9.1 \mathrm{mmol}, 1.3 \mathrm{eq})$

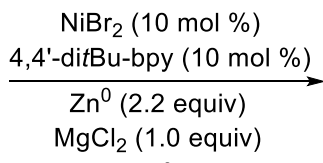

DMA, $80^{\circ} \mathrm{C}, 12 \mathrm{~h}$

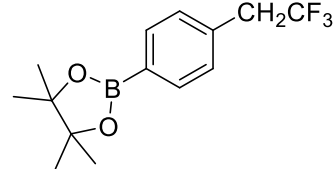

$22,87 \%$

$(6.1 \mathrm{mmol}, 1.74 \mathrm{~g})$

To a $100 \mathrm{~mL}$ of Schlenk tube were added compound $\mathbf{2 q}$ (1.3 equiv, $9.1 \mathrm{mmol}, 2.58 \mathrm{~g}), \mathrm{NiBr}_{2}(0.10$ equiv, $0.07 \mathrm{mmol}, 15.3 \mathrm{mg}), 4,4$-dtbbpy (0.10 equiv, $0.07 \mathrm{mmol}, 18.8 \mathrm{mg}$ ), $\mathrm{MgCl}_{2}$ (1.0 equiv, 7.0 mmol, $0.67 \mathrm{~g}$ ), and zinc ( 2.2 equiv, $15.4 \mathrm{mmol}, 1.00 \mathrm{~g}$ ). The tube was then evacuated and refilled with argon for three times. Anhydrous DMA $(40 \mathrm{~mL})$ and a solution of 1 in DMA (1.0 equiv, 7.0 mmol) were added under Ar. After stirring at $80^{\circ} \mathrm{C}$ for $12 \mathrm{~h}$, the reaction mixture was cooled to room temperature and quenched by water $(100 \mathrm{~mL})$ and ethyl acetate $(200 \mathrm{~mL})$. The reaction mixture was stirred for 5 minutes, and was washed by $0.5 \mathrm{M} \mathrm{HCl}(200 \mathrm{~mL})$ and brine $(200 \mathrm{~mL})$, successively. The organic layer was dried over anhydrous $\mathrm{Na}_{2} \mathrm{SO}_{4}$, filtered, and concentrated. The crude solid was purified by recrystallization (petroleum ether) to afford the pure product 22 as a white solid (6.1 mmol, $1.74 \mathrm{~g}, 87 \%$ yield).

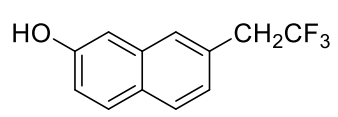

7-(2, 2, 2-Trifluoroethyl)naphthalen-2-ol (23). The reaction was conducted on $0.2 \mathrm{mmol}$ scale. The product 23 (ArX: $\mathrm{X}=\mathrm{Br}, 23 \mathrm{mg}, 50 \%$ yield) as a white solid (m.p. 98-99 ${ }^{\circ} \mathrm{C}$ ) was purified by silica gel column chromatography (PE:EA = 5:1). ${ }^{1} \mathrm{H}$ NMR (400 MHz, $\left.\mathrm{CDCl}_{3}\right): \delta 7.74(\mathrm{~d}, J=8.1 \mathrm{~Hz}, 2 \mathrm{H}), 7.58(\mathrm{~s}, 1 \mathrm{H}), 7.24(\mathrm{~d}, J=7.2 \mathrm{~Hz}, 1 \mathrm{H}), 7.14-7.08(\mathrm{~m}$, 2H), $5.14(\mathrm{~s}, 1 \mathrm{H}), 3.48(\mathrm{q}, J=10.7 \mathrm{~Hz}, 2 \mathrm{H}) .{ }^{13} \mathrm{C} \mathrm{NMR}\left(126 \mathrm{MHz}, \mathrm{CDCl}_{3}\right): \delta 153.8,134.5,129.7$, 128.4, $128.3\left(\mathrm{C}-\mathrm{F},{ }^{3} J_{\mathrm{C}-\mathrm{F}}=3.1 \mathrm{~Hz}\right), 128.2,127.9,125.9\left(\mathrm{C}-\mathrm{F},{ }^{1} J_{\mathrm{C}-\mathrm{F}}=277.0 \mathrm{~Hz}\right), 125.4,118.2,109.4$, $40.4\left(\mathrm{C}-\mathrm{F},{ }^{2} J_{\mathrm{C}-\mathrm{F}}=29.6 \mathrm{~Hz}\right) .{ }^{19} \mathrm{~F}$ NMR $\left(376 \mathrm{MHz}, \mathrm{CDCl}_{3}\right): \delta-65.6(\mathrm{t}, J=10.8 \mathrm{~Hz}, 3 \mathrm{~F}) . \mathrm{MS}(\mathrm{EI}) \mathrm{m} / \mathrm{z}$ : $226[\mathrm{M}]^{+}, 226$ (100). HRMS (EI-TOF) m/z: [M] ${ }^{+}$Calcd. for $\mathrm{C}_{12} \mathrm{H}_{9} \mathrm{~F}_{3} \mathrm{O}$ 226.0605; Found 226.0600.

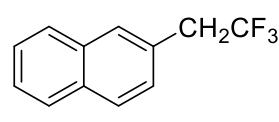

2-(2, 2, 2-Trifluoroethyl)naphthalene (24). The reaction was conducted on 0.2 mmol scale. The product $24(\mathrm{ArX}: \mathrm{X}=\mathrm{Br}, 34 \mathrm{mg}, 81 \%$ yield; $\mathrm{X}=\mathrm{Cl}, 31 \mathrm{mg}, 74 \%$ yield) as a white solid (m.p. $51-52{ }^{\circ} \mathrm{C}$ ) was purified by silica gel column chromatography (Petroleum ether). This compound is known. ${ }^{3} \mathrm{H}$ NMR $\left(400 \mathrm{MHz}, \mathrm{CDCl}_{3}\right)$ : $\delta$ 7.87-7.85 (m, 3H), $7.79(\mathrm{~s}, 1 \mathrm{H})$, 7.53-7.50 (m, 2H), $7.42(\mathrm{~d}, J=8.4 \mathrm{~Hz}, 1 \mathrm{H}), 3.55(\mathrm{q}, J=10.8 \mathrm{~Hz}, 2 \mathrm{H}) .{ }^{13} \mathrm{C} \mathrm{NMR}\left(126 \mathrm{MHz}, \mathrm{CDCl}_{3}\right)$ : 
$\delta 133.2,132.8,129.5,128.4,127.8,127.7,127.57,127.55\left(\mathrm{C}-\mathrm{F},{ }^{3} J_{\mathrm{C}-\mathrm{F}}=3.0 \mathrm{~Hz}\right), 126.4,126.3,125.9$ $\left(\mathrm{C}-\mathrm{F},{ }^{1} J_{\mathrm{C}-\mathrm{F}}=276.9 \mathrm{~Hz}\right), 40.4\left(\mathrm{C}-\mathrm{F},{ }^{2} J_{\mathrm{C}-\mathrm{F}}=29.7 \mathrm{~Hz}\right) .{ }^{19} \mathrm{~F} \mathrm{NMR}\left(376 \mathrm{MHz}, \mathrm{CDCl}_{3}\right): \delta-65.7(\mathrm{t}, J=10.8$ $\mathrm{Hz}, 3 \mathrm{~F}) . \mathrm{MS}(\mathrm{EI}) \mathrm{m} / \mathrm{z}: 210[\mathrm{M}]^{+}, 57$ (100). HRMS (EI-TOF) m/z: $[\mathrm{M}]^{+}$Calcd. for $\mathrm{C}_{12} \mathrm{H}_{9} \mathrm{~F}_{3} 210.0656$; Found 210.0654 .

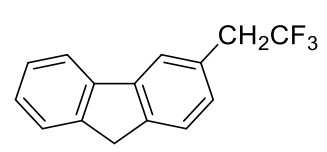

2-(2, 2, 2-Trifluoroethyl)-9H-fluorene (25). The product 25 (ArX: $\mathrm{X}=\mathrm{Br}, 65$ $\mathrm{mg}, 65 \%$ yield) as a white solid (m.p. $93-94{ }^{\circ} \mathrm{C}$ ) was purified by silica gel column chromatography (Petroleum ether). This compound is known. ${ }^{9}{ }^{1} \mathrm{H}$ NMR $\left(400 \mathrm{MHz}, \mathrm{CDCl}_{3}\right)$ : $\delta 7.81(\mathrm{~d}, J=7.5 \mathrm{~Hz}, 1 \mathrm{H}), 7.77(\mathrm{~d}, J=7.8 \mathrm{~Hz}, 1 \mathrm{H}), 7.57$ (d, $J=7.4 \mathrm{~Hz}, 1 \mathrm{H}), 7.49(\mathrm{~s}, 1 \mathrm{H}), 7.43(\mathrm{t}, J=$ $7.4 \mathrm{~Hz}, 1 \mathrm{H}), 7.37$ (dd, $J=7.4 \mathrm{~Hz}, 1.1 \mathrm{~Hz}, 1 \mathrm{H}), 7.33$ (d, $J=8.0 \mathrm{~Hz}, 1 \mathrm{H}) 3.90$ (s, 2H), 3.46 (q, $J=$ $10.9 \mathrm{~Hz}, 2 \mathrm{H}) .{ }^{13} \mathrm{C} \mathrm{NMR}\left(126 \mathrm{MHz}, \mathrm{CDCl}_{3}\right): \delta 143.7,143.3,141.6,141.1,128.8,128.4\left(\mathrm{C}-\mathrm{F},{ }^{3} J_{\mathrm{C}-\mathrm{F}}=\right.$ $2.9 \mathrm{~Hz}), 126.9,126.8,125.9\left(\mathrm{C}-\mathrm{F},{ }^{1} J_{\mathrm{C}-\mathrm{F}}=276.8 \mathrm{~Hz}\right), 125.0,120.00,119.9,40.3\left(\mathrm{C}-\mathrm{F},{ }^{2} J_{\mathrm{C}-\mathrm{F}}=29.6 \mathrm{~Hz}\right)$, 36.7. ${ }^{19}$ F NMR (376 MHz, $\left.\mathrm{CDCl}_{3}\right): \delta-65.8$ (t, $\left.J=10.9 \mathrm{~Hz}, 3 \mathrm{~F}\right)$. MS (EI) m/z: $248[\mathrm{M}]^{+}, 248(100)$. HRMS (EI-TOF) m/z: [M] Calcd. for $\mathrm{C}_{15} \mathrm{H}_{11} \mathrm{~F}_{3}$ 248.0813; Found 248.0819.

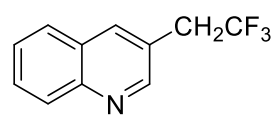

3-(2, 2, 2-Trifluoroethyl)quinolone (26). The product 26 (ArX: $\mathrm{X}=\mathrm{Br}, 31 \mathrm{mg}, \mathbf{4 0 \%}$ yield; $\mathrm{X}=\mathrm{Cl}, 53 \mathrm{mg}, 63 \%$ yield) as a white solid (m.p. $60-62{ }^{\circ} \mathrm{C}$ ) was purified by silica gel column chromatography $(\mathrm{PE}: \mathrm{EA}=4: 1)$. This compound is known. ${ }^{10} \mathrm{H}$ NMR $(400 \mathrm{MHz}$, $\left.\mathrm{CDCl}_{3}\right): \delta 8.83(\mathrm{~d}, J=1.8 \mathrm{~Hz}, 1 \mathrm{H}), 8.14-8.09(\mathrm{~m}, 2 \mathrm{H}), 7.81(\mathrm{~d}, J=8.1 \mathrm{~Hz}, 1 \mathrm{H}), 7.73(\mathrm{td}, J=6.8 \mathrm{~Hz}$, $1.6 \mathrm{~Hz}, 1 \mathrm{H}), 7.57(\mathrm{t}, J=7.5 \mathrm{~Hz}, 1 \mathrm{H}), 3.56(\mathrm{q}, J=10.6 \mathrm{~Hz}, 2 \mathrm{H}) .{ }^{13} \mathrm{C} \mathrm{NMR}\left(126 \mathrm{MHz}, \mathrm{CDCl}_{3}\right): \delta$ 151.5, 147.6, 137.3, 130.0, 129.2, 127.7, 127.6, $127.2,125.4\left(\mathrm{C}-\mathrm{F},{ }^{1} J_{\mathrm{C}-\mathrm{F}}=276.9 \mathrm{~Hz}\right), 123.1(\mathrm{C}-\mathrm{F}$, $\left.{ }^{3} J_{\mathrm{C}-\mathrm{F}}=2.8 \mathrm{~Hz}\right), 37.8\left(\mathrm{C}-\mathrm{F},{ }^{2} J_{\mathrm{C}-\mathrm{F}}=30.6 \mathrm{~Hz}\right) .{ }^{19} \mathrm{~F} \mathrm{NMR}\left(376 \mathrm{MHz}, \mathrm{CDCl}_{3}\right): \delta-65.8(\mathrm{t}, J=10.6 \mathrm{~Hz}, 3 \mathrm{~F})$. MS (EI) m/z: $211[\mathrm{M}]^{+}, 142$ (100). HRMS (EI-TOF) m/z: [M] $]^{+}$Calcd. for $\mathrm{C}_{11} \mathrm{H}_{8} \mathrm{~F}_{3} \mathrm{~N} 211.0609$; Found 211.0608.

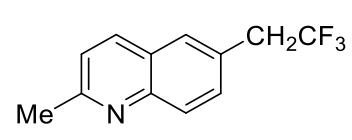

2-Methyl-6-(2, 2, 2-trifluoroethyl)quinolone (27). The product 27 (ArX: $\mathrm{X}=$ $\mathrm{Br}, 63 \mathrm{mg}, 70 \%$ yield; $\mathrm{X}=\mathrm{Cl}, 65 \mathrm{mg}, 72 \%$ yield) as a white solid (m.p. $87-88{ }^{\circ} \mathrm{C}$ ) was purified by silica gel column chromatography (PE:EA $\left.=4: 1\right) .{ }^{1} \mathrm{H}$ NMR $(400 \mathrm{MHz}$, $\left.\mathrm{CDCl}_{3}\right): \delta 8.02(\mathrm{t}, J=8.0 \mathrm{~Hz}, 2 \mathrm{H}), 7.70(\mathrm{~s}, 1 \mathrm{H}), 7.60(\mathrm{~d}, J=8.8 \mathrm{~Hz}, 1 \mathrm{H}), 7.31(\mathrm{~d}, J=8.5 \mathrm{~Hz}, 1 \mathrm{H})$, 
$3.54(\mathrm{q}, J=10.7 \mathrm{~Hz}, 2 \mathrm{H}), 2.75$ (s, 3H). ${ }^{13} \mathrm{C} \mathrm{NMR}\left(126 \mathrm{MHz}, \mathrm{CDCl}_{3}\right): \delta 159.5,147.3,135.9,131.1$, $129.1,129.0,127.4\left(\mathrm{C}-\mathrm{F},{ }^{3} J_{\mathrm{C}-\mathrm{F}}=3.0 \mathrm{~Hz}\right), 126.2,125.7\left(\mathrm{C}-\mathrm{F},{ }^{1} J_{\mathrm{C}-\mathrm{F}}=277.0 \mathrm{~Hz}\right), 122.4,40.0\left(\mathrm{C}-\mathrm{F},{ }^{2} J_{\mathrm{C}-\mathrm{F}}\right.$ $=29.8 \mathrm{~Hz}), 25.3 .{ }^{19} \mathrm{~F}$ NMR $\left(376 \mathrm{MHz}, \mathrm{CDCl}_{3}\right): \delta-65.8(\mathrm{t}, J=10.7 \mathrm{~Hz}, 3 \mathrm{~F}) . \mathrm{MS}(\mathrm{EI}) \mathrm{m} / \mathrm{z}: 225[\mathrm{M}]^{+}$, 156 (100). HRMS (EI-TOF) m/z: [M] ${ }^{+}$Calcd. for $\mathrm{C}_{12} \mathrm{H}_{10} \mathrm{~F}_{3} \mathrm{~N} 225.0765$; Found 225.0761.

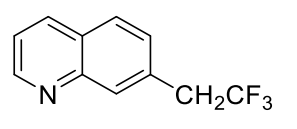

7-(2, 2, 2-trifluoroethyl)quinoline (28). The reaction was conducted on 0.2 $35 \mathrm{mg}, 82 \%$ yield) as a white solid (m.p. $58-59{ }^{\circ} \mathrm{C}$ ) was purified by silica gel column chromatography (PE:EA = 4:1). ${ }^{1} \mathrm{H}$ NMR $\left(400 \mathrm{MHz}, \mathrm{CDCl}_{3}\right): \delta 8.91(\mathrm{dd}, J=2.7 \mathrm{~Hz}, 1.4 \mathrm{~Hz}, 1 \mathrm{H})$, $8.11(\mathrm{~d}, J=8.2 \mathrm{~Hz}, 1 \mathrm{H}), 8.04(\mathrm{~s}, 1 \mathrm{H}), 7.78(\mathrm{~d}, J=8.4 \mathrm{~Hz}, 1 \mathrm{H}), 7.45(\mathrm{~d}, J=8.3 \mathrm{~Hz}, 1 \mathrm{H}), 7.37$ (dd, $J=$ $8.3 \mathrm{~Hz}, 4.2 \mathrm{~Hz}, 1 \mathrm{H}), 3.57$ (q, $J=10.7 \mathrm{~Hz}, 2 \mathrm{H}) .{ }^{13} \mathrm{C} \mathrm{NMR}\left(126 \mathrm{MHz}, \mathrm{CDCl}_{3}\right): \delta 150.9,148.0,135.7$, $131.5\left(\mathrm{C}-\mathrm{F},{ }^{3} J_{\mathrm{C}-\mathrm{F}}=2.9 \mathrm{~Hz}\right), 131.1,128.18,128.14,127.6,125.6\left(\mathrm{C}-\mathrm{F},{ }^{1} J_{\mathrm{C}-\mathrm{F}}=277.1 \mathrm{~Hz}\right), 121.4,40.3$ $\left(\mathrm{C}-\mathrm{F},{ }^{2} J_{\mathrm{C}-\mathrm{F}}=29.9 \mathrm{~Hz}\right) .{ }^{19} \mathrm{~F}$ NMR $\left(376 \mathrm{MHz}, \mathrm{CDCl}_{3}\right): \delta-65.5(\mathrm{t}, J=10.7 \mathrm{~Hz}, 3 \mathrm{~F}) . \mathrm{MS}(\mathrm{EI}) \mathrm{m} / \mathrm{z}: 211$ $[\mathrm{M}]^{+}, 211$ (100). HRMS (EI-TOF) m/z: [M] $]^{+}$Calcd. for $\mathrm{C}_{11} \mathrm{H}_{8} \mathrm{~F}_{3} \mathrm{~N} 211.0609$; Found 211.0613.

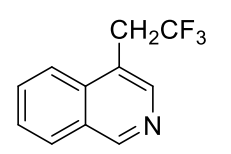

4-(2, 2, 2-Trifluoroethyl)isoquinoline (29). The product 29 (ArX: $\mathrm{X}=\mathrm{Br}, 52 \mathrm{mg}, 62 \%$ yield; $\mathrm{X}=\mathrm{Cl}, 48 \mathrm{mg}, 57 \%$ yield) as a white solid (m.p. $72-73{ }^{\circ} \mathrm{C}$ ) was purified by silica gel column chromatography (PE:EA = 4:1). ${ }^{1} \mathrm{H} \mathrm{NMR}\left(400 \mathrm{MHz}, \mathrm{CDCl}_{3}\right): \delta 9.26(\mathrm{~s}, 1 \mathrm{H}), 8.51(\mathrm{~s}, 1 \mathrm{H})$, $8.03(\mathrm{~d}, J=8.2 \mathrm{~Hz}, 1 \mathrm{H}), 7.99(\mathrm{~d}, J=8.5 \mathrm{~Hz}, 1 \mathrm{H}), 7.81(\mathrm{t}, J=7.7 \mathrm{~Hz}, 1 \mathrm{H}), 7.67(\mathrm{t}, J=7.5 \mathrm{~Hz}, 1 \mathrm{H})$, $3.82(\mathrm{q}, J=10.4 \mathrm{~Hz}, 2 \mathrm{H}) .{ }^{13} \mathrm{C} \mathrm{NMR}\left(126 \mathrm{MHz}, \mathrm{CDCl}_{3}\right): \delta 153.5,145.4,135.0,131.1,128.4,127.4$, $125.7\left(\mathrm{C}-\mathrm{F},{ }^{1} J_{\mathrm{C}-\mathrm{F}}=277.6 \mathrm{~Hz}\right), 122.7\left(\mathrm{C}-\mathrm{F},{ }^{3} J_{\mathrm{C}-\mathrm{F}}=1.3 \mathrm{~Hz}\right), 120.2,110.0,34.4\left(\mathrm{C}-\mathrm{F},{ }^{2} J_{\mathrm{C}-\mathrm{F}}=30.9 \mathrm{~Hz}\right)$. ${ }^{19} \mathrm{~F} \mathrm{NMR}\left(376 \mathrm{MHz}, \mathrm{CDCl}_{3}\right): \delta-64.7$ (t, $\left.J=10.4 \mathrm{~Hz}, 3 \mathrm{~F}\right) . \mathrm{MS}(\mathrm{EI}) \mathrm{m} / \mathrm{z}: 211[\mathrm{M}]^{+}, 211(100)$. HRMS (EI-TOF) m/z: [M] $]^{+}$Calcd. for $\mathrm{C}_{11} \mathrm{H}_{8} \mathrm{~F}_{3} \mathrm{~N}$ 211.0609; Found 211.0603.

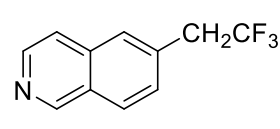

6-(2, 2, 2-Trifluoroethyl)isoquinoline (30). The product $30(\mathrm{ArX}: \mathrm{X}=\mathrm{Br}, 51 \mathrm{mg}$, $60 \%$ yield; $\mathrm{X}=\mathrm{Cl}, 46 \mathrm{mg}, 55 \%$ yield) as a yellow oil was purified by silica gel column chromatography (PE:EA = 4:1). ${ }^{1} \mathrm{H}$ NMR (400 MHz, $\left.\mathrm{CDCl}_{3}\right): \delta 9.24(\mathrm{~s}, 1 \mathrm{H}), 8.53(\mathrm{~d}, J=5.8$ $\mathrm{Hz}, 1 \mathrm{H}), 7.95(\mathrm{~d}, J=8.4 \mathrm{~Hz}, 1 \mathrm{H}), 7.74(\mathrm{~s}, 1 \mathrm{H}), 7.62(\mathrm{~d}, J=5.8 \mathrm{~Hz}, 1 \mathrm{H}), 7.52$ (d, $J=8.4 \mathrm{~Hz}, 1 \mathrm{H})$, $3.55(\mathrm{q}, J=10.6 \mathrm{~Hz}, 2 \mathrm{H}) .{ }^{13} \mathrm{C} \mathrm{NMR}\left(126 \mathrm{MHz}, \mathrm{CDCl}_{3}\right): \delta 152.2,143.5,135.6,132.5\left(\mathrm{C}-\mathrm{F},{ }^{3} J_{\mathrm{C}-\mathrm{F}}=\right.$ 
$2.9 \mathrm{~Hz}), 129.0,128.1,128.00,125.5\left(\mathrm{C}-\mathrm{F},{ }^{1} J_{\mathrm{C}-\mathrm{F}}=277.1 \mathrm{~Hz}\right), 120.3,110.0,40.5\left(\mathrm{C}-\mathrm{F},{ }^{2} J_{\mathrm{C}-\mathrm{F}}=29.9 \mathrm{~Hz}\right)$. ${ }^{19} \mathrm{~F} \mathrm{NMR}\left(376 \mathrm{MHz}, \mathrm{CDCl}_{3}\right): \delta-65.4(\mathrm{t}, J=10.6 \mathrm{~Hz}, 3 \mathrm{~F}) . \mathrm{MS}(\mathrm{EI}) \mathrm{m} / \mathrm{z}: 211[\mathrm{M}]^{+}, 211(100)$. HRMS (EI-TOF) m/z: [M] $]^{+}$Calcd. for $\mathrm{C}_{11} \mathrm{H}_{8} \mathrm{~F}_{3} \mathrm{~N} 211.0609$; Found 211.0604 .

$\mathrm{CH}_{2} \mathrm{CF}_{3}$

Tert-butyl 4-(2, 2, 2-trifluoroethyl)-1H-indole-1-carboxylate (31). The product 31 (ArX: $\mathrm{X}=\mathrm{Br}, 92 \mathrm{mg}, 77 \%$ yield) as a yellow oil was purified by silica gel column Boc chromatography (PE:EA $=10: 1)$. This compound is known. ${ }^{11}{ }^{1} \mathrm{H}$ NMR $(400 \mathrm{MHz}$, $\left.\mathrm{CDCl}_{3}\right): \delta 8.17(\mathrm{~d}, J=8.2 \mathrm{~Hz}, 1 \mathrm{H}), 7.66(\mathrm{~d}, J=3.7 \mathrm{~Hz}, 1 \mathrm{H}), 7.31(\mathrm{t}, J=7.9 \mathrm{~Hz}, 1 \mathrm{H}), 7.18(\mathrm{~d}, J=7.4$ $\mathrm{Hz}, 1 \mathrm{H}), 6.63(\mathrm{~d}, J=3.7 \mathrm{~Hz}, 1 \mathrm{H}), 3.62$ (q, $J=10.8 \mathrm{~Hz}, 2 \mathrm{H}), 1.68(\mathrm{~s}, 9 \mathrm{H}) .{ }^{13} \mathrm{C}$ NMR (126 MHz, $\left.\mathrm{CDCl}_{3}\right): \delta 149.6,135.3,130.8,126.4,126.0\left(\mathrm{C}-\mathrm{F},{ }^{1} J_{\mathrm{C}-\mathrm{F}}=277.3 \mathrm{~Hz}\right), 124.8,124.3,122.2\left(\mathrm{C}-\mathrm{F},{ }^{3} J_{\mathrm{C}-\mathrm{F}}=\right.$ $3.1 \mathrm{~Hz}), 115.2,105.1,83.9,37.5\left(\mathrm{C}-\mathrm{F},{ }^{2} J_{\mathrm{C}-\mathrm{F}}=30.2 \mathrm{~Hz}\right), 28.2 .{ }^{19} \mathrm{~F} \mathrm{NMR}\left(376 \mathrm{MHz}, \mathrm{CDCl}_{3}\right): \delta-65.3(\mathrm{t}$, $J=10.8 \mathrm{~Hz}, 3 \mathrm{~F}$ ). MS (EI) m/z: $299[\mathrm{M}]^{+}, 57$ (100). HRMS (EI-TOF) m/z: $[\mathrm{M}]^{+}$Calcd. for $\mathrm{C}_{15} \mathrm{H}_{16} \mathrm{~F}_{3} \mathrm{NO}_{2} 299.1133$; Found 299.1126.

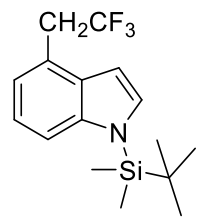

1-(Tert-butyldimethylsilyl)-4-(2,2,2-trifluoroethyl)-1H-indole (32). The reaction was conducted on $0.2 \mathrm{mmol}$ scale for $20 \mathrm{~h}$. The product 32 (ArX: X= Br, $52 \mathrm{mg}, 83 \%$ yield) as a brown solid (m.p. $57-58{ }^{\circ} \mathrm{C}$ ) was purified by silica gel column chromatography $(\mathrm{PE}: \mathrm{EA}=10: 1) .{ }^{1} \mathrm{H} \mathrm{NMR}\left(400 \mathrm{MHz}, \mathrm{CDCl}_{3}\right): \delta 7.53(\mathrm{~d}, J=8.2 \mathrm{~Hz}, 1 \mathrm{H}), 7.27(\mathrm{~d}, J=3.5 \mathrm{~Hz}, 1 \mathrm{H})$, $7.17(\mathrm{t}, J=7.7 \mathrm{~Hz}, 1 \mathrm{H}), 7.09(\mathrm{~d}, J=7.2 \mathrm{~Hz}, 1 \mathrm{H}), 6.68(\mathrm{~d}, J=2.4 \mathrm{~Hz}, 1 \mathrm{H}), 3.67(\mathrm{q}, J=10.9 \mathrm{~Hz}, 2 \mathrm{H})$, $0.96(\mathrm{~s}, 9 \mathrm{H}), 0.63(\mathrm{~s}, 6 \mathrm{H}) .{ }^{13} \mathrm{C} \mathrm{NMR}\left(126 \mathrm{MHz} \mathrm{CDCl}_{3}\right): \delta 141.0,131.7,131.5,126.4\left(\mathrm{C}-\mathrm{F},{ }^{1} J_{\mathrm{C}-\mathrm{F}}=\right.$ $277.4 \mathrm{~Hz}), 121.9,121.8\left(\mathrm{C}-\mathrm{F},{ }^{3} J_{\mathrm{C}-\mathrm{F}}=2.9 \mathrm{~Hz}\right), 121.3,113.8,102.9,38.0\left(\mathrm{C}-\mathrm{F},{ }^{2} J_{\mathrm{C}-\mathrm{F}}=30.0 \mathrm{~Hz}\right), 26.3$, 19.5, -4.0. ${ }^{19} \mathrm{~F}$ NMR $\left(376 \mathrm{MHz}, \mathrm{CDCl}_{3}\right): \delta-65.0$ (t, $\left.\left.J=10.9 \mathrm{~Hz}, 3 \mathrm{~F}\right) . \mathrm{MS}(\mathrm{EI}) \mathrm{m} / \mathrm{z}: 313[\mathrm{M}]\right]^{+}, 313$ (100). HRMS (EI-TOF) m/z: [M] ${ }^{+}$Calcd. for $\mathrm{C}_{16} \mathrm{H}_{22} \mathrm{~F}_{3} \mathrm{NSi}$ 313.1474; Found 313.1481.

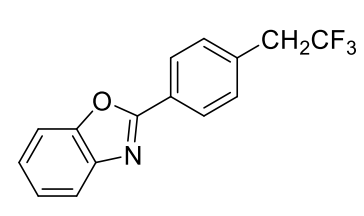

2-(4-(2, 2, 2-Trifluoroethyl)phenyl)benzo[d]oxazole (33). The product 33 (ArX: $\mathrm{X}=\mathrm{Br}, 46 \mathrm{mg}, 51 \%$ yield) was a white solid (m.p. $137-138^{\circ} \mathrm{C}$ ) purified by silica gel column chromatography $(\mathrm{PE}: \mathrm{EA}=10: 1) .{ }^{1} \mathrm{H}$ NMR $(400 \mathrm{MHz}$, $\left.\mathrm{CDCl}_{3}\right): \delta 8.25(\mathrm{~d}, J=8.3 \mathrm{~Hz}, 2 \mathrm{H}), 7.80-7.76(\mathrm{~m}, 1 \mathrm{H}), 7.60-7.58(\mathrm{~m}, 1 \mathrm{H}), 7.47(\mathrm{~d}, J=8.1 \mathrm{~Hz}, 2 \mathrm{H})$, 7.39-7.36 (m, 2H), 3.46 (q, $J=10.7 \mathrm{~Hz}, 2 \mathrm{H}) .{ }^{13} \mathrm{C} \mathrm{NMR}\left(126 \mathrm{MHz}, \mathrm{CDCl}_{3}\right): \delta 162.5,150.8,142.0$, 
$133.6,130.7,127.9,127.1,125.5\left(\mathrm{C}-\mathrm{F},{ }^{1} J_{\mathrm{C}-\mathrm{F}}=277.0 \mathrm{~Hz}\right), 125.3,124.7,120.1,110.6,40.2\left(\mathrm{C}-\mathrm{F},{ }^{2} J_{\mathrm{C}-\mathrm{F}}\right.$ $=30.4 \mathrm{~Hz}) .{ }^{19} \mathrm{~F}$ NMR $\left(376 \mathrm{MHz}, \mathrm{CDCl}_{3}\right): \delta-65.6$ (t, $\left.J=10.7 \mathrm{~Hz}, 3 \mathrm{~F}\right) . \mathrm{MS}(\mathrm{EI}) \mathrm{m} / \mathrm{z}: 277[\mathrm{M}]^{+}, 277$ (100). HRMS (EI-TOF) m/z: [M] $]^{+}$Calcd. for $\mathrm{C}_{15} \mathrm{H}_{10} \mathrm{~F}_{3} \mathrm{NO} 277.0714$; Found 277.0710.

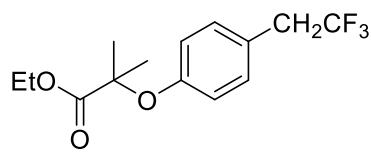

Ethyl 2-methyl-2-(4-(2, 2, 2-trifluoroethyl)phenoxy)propanoate (35a).

The product 35a (ArX: $\mathrm{X}=\mathrm{Cl}, 81 \mathrm{mg}, 70 \%$ yeild) as a yellow oil was purified by silica gel column chromatography $(\mathrm{PE}: \mathrm{EA}=20: 1)$. This compound is known. ${ }^{3}{ }^{1} \mathrm{H}$ NMR (400 MHz, $\left.\mathrm{CDCl}_{3}\right): \delta 7.15(\mathrm{~d}, J=8.4 \mathrm{~Hz}, 2 \mathrm{H}), 6.81(\mathrm{~d}, J=8.6 \mathrm{~Hz}, 2 \mathrm{H}), 4.23(\mathrm{q}, J=7.1 \mathrm{~Hz}, 2 \mathrm{H})$, $3.28(\mathrm{~d}, J=10.8 \mathrm{~Hz}, 2 \mathrm{H}), 1.60(\mathrm{~s}, 6 \mathrm{H}), 1.23(\mathrm{t}, J=7.1 \mathrm{~Hz}, 3 \mathrm{H}) .{ }^{13} \mathrm{C} \mathrm{NMR}\left(126 \mathrm{MHz}, \mathrm{CDCl}_{3}\right): \delta$ 174.1, 155.4, 130.9, $125.8\left(\mathrm{C}-\mathrm{F},{ }^{1} J_{\mathrm{C}-\mathrm{F}}=276.9 \mathrm{~Hz}\right), 123.5\left(\mathrm{C}-\mathrm{F},{ }^{3} J_{\mathrm{C}-\mathrm{F}}=3.0 \mathrm{~Hz}\right), 119.0,79.1,61.4,39.4$ $\left(\mathrm{C}-\mathrm{F},{ }^{2} J_{\mathrm{C}-\mathrm{F}}=29.7 \mathrm{~Hz}\right), 25.3,14.0 .{ }^{19} \mathrm{~F} \mathrm{NMR}\left(376 \mathrm{MHz}, \mathrm{CDCl}_{3}\right): \delta-65.6(\mathrm{t}, J=10.7 \mathrm{~Hz}, 3 \mathrm{~F}) . \mathrm{MS}(\mathrm{EI})$ m/z: $290[\mathrm{M}]^{+}, 176$ (100). HRMS (EI-TOF) m/z: [M] ${ }^{+}$Calcd. for $\mathrm{C}_{14} \mathrm{H}_{17} \mathrm{~F}_{3} \mathrm{O}_{3}$ 290.1130; Found 290.1126 .

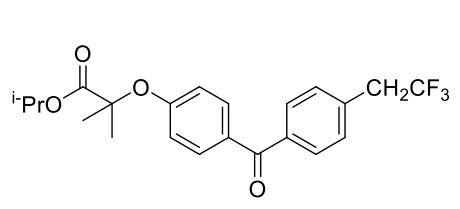

\section{Isopropyl}

2-methyl-2-(4-(4-(2,

2,

2-trifluoroethyl)benzoyl)phenoxy)propanoate (35b). The product $35 b$

(ArX: $\mathrm{X}=\mathrm{Cl} ; 139 \mathrm{mg}, 85 \%$ yeild) as a yellow solid (m.p. $60-61{ }^{\circ} \mathrm{C}$ ) was purified by silica gel column chromatography $(\mathrm{PE}: \mathrm{EA}=10: 1)$. This compound is known. ${ }^{3} \mathrm{H}$ NMR (400 MHz, $\left.\mathrm{CDCl}_{3}\right): \delta 7.74(\mathrm{t}, J=8.3 \mathrm{~Hz}, 4 \mathrm{H}), 7.40(\mathrm{~d}, J=7.9 \mathrm{~Hz}, 2 \mathrm{H}), 6.86(\mathrm{~d}, J=8.8 \mathrm{~Hz}, 2 \mathrm{H}), 5.08$ $(\mathrm{d}, J=6.0 \mathrm{~Hz}, 1 \mathrm{H}), 3.44(\mathrm{q}, J=10.7 \mathrm{~Hz}, 2 \mathrm{H}), 1.65(\mathrm{~s}, 6 \mathrm{H}), 1.19(\mathrm{~d}, J=6.4 \mathrm{~Hz}, 6 \mathrm{H}) .{ }^{13} \mathrm{C} \mathrm{NMR}(126$ $\left.\mathrm{MHz}, \mathrm{CDCl}_{3}\right): \delta 194.8,173.0,159.7,137.9,134.0\left(\mathrm{C}-\mathrm{F},{ }^{3} J_{\mathrm{C}-\mathrm{F}}=2.7 \mathrm{~Hz}\right), 132.0,130.3,130.00,129.97$, $125.5\left(\mathrm{C}-\mathrm{F},{ }^{1} J_{\mathrm{C}-\mathrm{F}}=277.0 \mathrm{~Hz}\right), 117.2,79.4,69.3,40.1\left(\mathrm{C}-\mathrm{F},{ }^{2} J_{\mathrm{C}-\mathrm{F}}=29.9 \mathrm{~Hz}\right), 25.3,21.5 .{ }^{19} \mathrm{~F}$ NMR (376 MHz, $\mathrm{CDCl}_{3}$ ): $\delta-65.6(\mathrm{t}, J=10.7 \mathrm{~Hz}, 3 \mathrm{~F}) . \mathrm{MS}(\mathrm{EI}) \mathrm{m} / \mathrm{z}: 408[\mathrm{M}]^{+}, 121$ (100). HRMS (EI-TOF) $\mathrm{m} / \mathrm{z}$ : $[\mathrm{M}]^{+}$Calcd. for $\mathrm{C}_{22} \mathrm{H}_{23} \mathrm{~F}_{3} \mathrm{O}_{4} 408.1548$; Found 408.1554 .

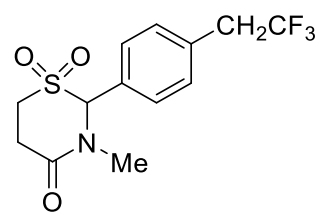

3-Methyl-2-(4-(2, 2， 2-trifluoroethyl)phenyl)-1， 3-thiazinan-4-one 1, 1-dioxide (35c). The reaction run for $20 \mathrm{~h}$. The product 35c $(\mathrm{ArX}: \mathrm{X}=\mathrm{Cl}, 90 \mathrm{mg}$, $70 \%$ yeild) as a yellow solid (m.p. $42-44{ }^{\circ} \mathrm{C}$ ) was purified by silica gel column chromatography (PE:EA = 1:1). ${ }^{1} \mathrm{H}$ NMR (400 MHz, $\left.\mathrm{CDCl}_{3}\right): \delta 7.41-7.34(\mathrm{~m}, 4 \mathrm{H}), 5.33(\mathrm{~s}, 1 \mathrm{H}), 3.39$ 
$(\mathrm{q}, J=10.7 \mathrm{~Hz}, 2 \mathrm{H}), 3.33-3.23(\mathrm{~m}, 1 \mathrm{H}), 3.20-3.01(\mathrm{~m}, 3 \mathrm{H}), 2.91(\mathrm{~s}, 3 \mathrm{H}) .{ }^{13} \mathrm{C} \mathrm{NMR}(126 \mathrm{MHz}$, $\left.\mathrm{CDCl}_{3}\right): \delta 166.1,132.5\left(\mathrm{C}-\mathrm{F},{ }^{3} J_{\mathrm{C}-\mathrm{F}}=2.9 \mathrm{~Hz}\right), 130.9,130.0,128.4,125.3\left(\mathrm{C}-\mathrm{F},{ }^{1} J_{\mathrm{C}-\mathrm{F}}=277.0 \mathrm{~Hz}\right), 80.0$, 43.3, $39.7\left(\mathrm{C}-\mathrm{F},{ }^{2} J_{\mathrm{C}-\mathrm{F}}=30.0 \mathrm{~Hz}\right), 36.0,30.4 .{ }^{19} \mathrm{~F} \mathrm{NMR}\left(376 \mathrm{MHz}, \mathrm{CDCl}_{3}\right): \delta-65.6(\mathrm{t}, J=10.7 \mathrm{~Hz}$, 3F). MS (ESI) m/z: $322[\mathrm{M}+\mathrm{H}]^{+}, 322$ (100). HRMS (ESI-TOF) m/z: $[\mathrm{M}+\mathrm{H}]^{+}$Calcd. for $\mathrm{C}_{13} \mathrm{H}_{15} \mathrm{~F}_{3} \mathrm{NO}_{3} \mathrm{~S}$ 322.0719; Found 322.0721.

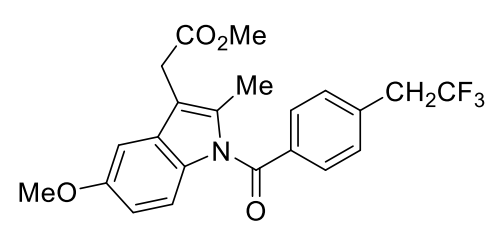

Methyl

2-(2-methyl-1-(4-(2,

2,

2-trifluoroethyl)benzoyl)-1H-indol-3-yl)acetate (35d). The product 35d (ArX: $\mathrm{X}=\mathrm{Cl}, 128 \mathrm{mg}, 80 \%$ yeild) as a solid (m.p. $85-86^{\circ} \mathrm{C}$ ) was purified by silica gel column chromatography $(\mathrm{PE}: \mathrm{EA}=8: 1) .{ }^{1} \mathrm{H} \mathrm{NMR}\left(400 \mathrm{MHz}, \mathrm{CDCl}_{3}\right): \delta 7.71(\mathrm{~d}$, $J=8.0 \mathrm{~Hz}, 2 \mathrm{H}), 7.43(\mathrm{~d}, J=7.9 \mathrm{~Hz}, 2 \mathrm{H}), 6.96(\mathrm{~d}, J=2.3 \mathrm{~Hz}, 1 \mathrm{H}), 6.89$ (d, $J=9.0 \mathrm{~Hz}, 1 \mathrm{H}), 6.66(\mathrm{dd}$, $J=9.0 \mathrm{~Hz}, J=2.4 \mathrm{~Hz}, 1 \mathrm{H}), 3.84(\mathrm{~s}, 3 \mathrm{H}), 3.71(\mathrm{~s} 3 \mathrm{H}), 3.67(\mathrm{~s}, 2 \mathrm{H}), 3.48$ (q, $J=10.6 \mathrm{~Hz}, 2 \mathrm{H}), 2.37$ (s, $3 \mathrm{H}) .{ }^{13} \mathrm{C} \mathrm{NMR}\left(126 \mathrm{MHz}, \mathrm{CDCl}_{3}\right): \delta 171.3,168.8,156.0,135.9,135.5,135.0,130.9,130.64,130.56$, $130.0,125.4\left(\mathrm{C}-\mathrm{F},{ }^{1} J_{\mathrm{C}-\mathrm{F}}=277.6 \mathrm{~Hz}\right), 115.0,112.4,111.6,101.3,55.7,52.2,40.2\left(\mathrm{C}-\mathrm{F},{ }^{2} J_{\mathrm{C}-\mathrm{F}}=30.1\right.$ $\mathrm{Hz}), 30.1,13.4 .{ }^{19} \mathrm{~F}$ NMR $\left(376 \mathrm{MHz}, \mathrm{CDCl}_{3}\right): \delta-65.8(\mathrm{t}, J=11.6 \mathrm{~Hz}, 3 \mathrm{~F}) . \mathrm{MS}(\mathrm{EI}) \mathrm{m} / \mathrm{z}: 419[\mathrm{M}]^{+}$, 187 (100). HRMS (EI-TOF) m/z: [M] Calcd. for $\mathrm{C}_{22} \mathrm{H}_{20} \mathrm{~F}_{3} \mathrm{NO}_{4} 419.1344$; Found 419.1348.

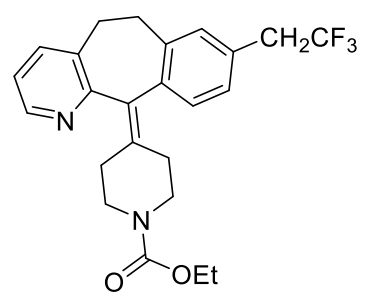

Ethyl 4-(8-(2, 2, 2-trifluoroethyl)-5, 6-dihydro-11H-benzo[5, 6]cyclohepta[1，2-b]pyridin-11-ylidene)piperidine-1-carboxylate (35e). The product 35e $(\mathrm{ArX}: \mathrm{X}=\mathrm{Cl}, 134 \mathrm{mg}, 78 \%$ yeild) as a white solid (m.p. $\left.48-50{ }^{\circ} \mathrm{C}\right)$ was purified by silica gel column chromatography $(\mathrm{PE}: \mathrm{EA}=1: 1)$. This compound is known. ${ }^{12}{ }^{1} \mathrm{H}$ NMR $\left(400 \mathrm{MHz}, \mathrm{CDCl}_{3}\right): \delta 8.37(\mathrm{~d}, J=3.9 \mathrm{~Hz}, 1 \mathrm{H}), 7.40(\mathrm{~d}, J=7.4$ $\mathrm{Hz}, 1 \mathrm{H}), 7.17-7.02(\mathrm{~m}, 4 \mathrm{H}), 4.10(\mathrm{q}, J=7.2 \mathrm{~Hz}, 2 \mathrm{H}), 3.80(\mathrm{~s}, 2 \mathrm{H}), 3.46-3.27(\mathrm{~m}, 2 \mathrm{H}), 3.27(\mathrm{q}, J=$ $10.8 \mathrm{~Hz}, 2 \mathrm{H}), 3.14-3.07$ (m, 2H), 2.84-2.78 (m, 2H), 2.49-2.43 (m, 1H), 2.39-2.28 (m, 3H), 1.21 (t, $J$ $=7.1 \mathrm{~Hz}, 3 \mathrm{H}) .{ }^{13} \mathrm{C} \mathrm{NMR}\left(126 \mathrm{MHz}, \mathrm{CDCl}_{3}\right): \delta 157.3,155.5,146.6,139.0,138.0,137.4,137.1,134.7$, 133.5, 130.8, 129.6, $129.1\left(\mathrm{C}-\mathrm{F},{ }^{3} J_{\mathrm{C}-\mathrm{F}}=2.5 \mathrm{~Hz}\right), 127.8,125.7\left(\mathrm{C}-\mathrm{F},{ }^{1} J_{\mathrm{C}-\mathrm{F}}=277.5 \mathrm{~Hz}\right), 122.2,61.3$, $44.83,44.76,39.8\left(\mathrm{C}-\mathrm{F},{ }^{2} J_{\mathrm{C}-\mathrm{F}}=29.7 \mathrm{~Hz}\right), 31.8,31.6,30.5,29.7,14.6 .{ }^{19} \mathrm{~F} \mathrm{NMR}\left(376 \mathrm{MHz}, \mathrm{CDCl}_{3}\right): \delta$ $-65.8(\mathrm{t}, J=10.8 \mathrm{~Hz}, 3 \mathrm{~F})$. MS (EI) m/z: $430[\mathrm{M}]^{+}, 430$ (100). HRMS (EI-TOF) m/z: [M] $]^{+}$Calcd. for $\mathrm{C}_{24} \mathrm{H}_{25} \mathrm{~F}_{3} \mathrm{~N}_{2} \mathrm{O}_{2} 430.1868$; Found 430.1873 . 


\section{Transformations of Compound 22}

Synthesis of compound $37^{13}$
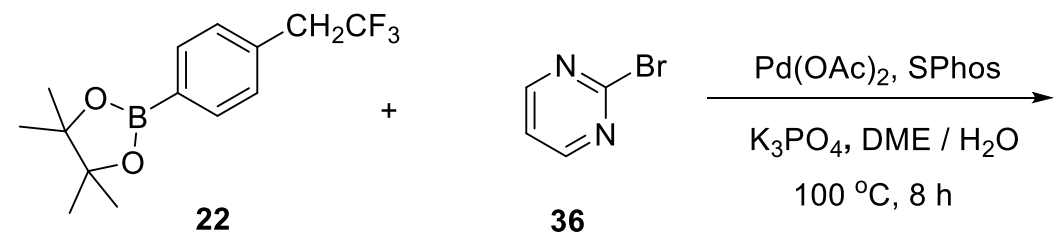<smiles>FC(F)(F)Cc1ccc(-c2ncccn2)cc1</smiles>

$37,80 \%$

To a $25 \mathrm{~mL}$ of Schlenk tube were added compound 22 (286.1 mg, $0.33 \mathrm{mmol}$, 1.1 equiv), 2-bromopyrimidine 36 (47.7 mg, $0.3 \mathrm{mmol}, 1.0$ equiv), $\mathrm{Pd}(\mathrm{OAc})_{2}(0.7 \mathrm{mg}, 0.003 \mathrm{mmol}, 0.01$ equiv), S-Phos (2.5 mg, 0.006 mmol, 0.02 equiv) and $\mathrm{K}_{3} \mathrm{PO}_{4}(191.0 \mathrm{mg}, 0.9 \mathrm{mmol}, 3.0$ equiv). The tube was evacuated and refilled with argon for three times. 1,2-Dimethoxyethane $(1 \mathrm{~mL})$ and $\mathrm{H}_{2} \mathrm{O}(1 \mathrm{~mL})$ were added. After stirring for $8 \mathrm{~h}$ at $100{ }^{\circ} \mathrm{C}$, the reaction mixture was cooled to room temperature, and diluted with ethyl acetate $(100 \mathrm{~mL})$. The resulting mixture was washed by water and brine, dried over anhydrous $\mathrm{Na}_{2} \mathrm{SO}_{4}$, filtered, and concentrated. The residue was purified with flash chromatography on silica gel (petroleum ether: ethyl acetate $=2: 1)$ to give product $37(57 \mathrm{mg}, 80 \%$ yield) as a white solid (m.p. $\left.82-83{ }^{\circ} \mathrm{C}\right) .{ }^{1} \mathrm{H}$ NMR (400 MHz, $\left.\mathrm{CDCl}_{3}\right): \delta 8.81(\mathrm{~d}, J=4.8 \mathrm{~Hz}, 2 \mathrm{H}), 8.44$ $(\mathrm{d}, J=8.3 \mathrm{~Hz}, 2 \mathrm{H}), 7.43(\mathrm{~d}, J=8.1 \mathrm{~Hz}, 2 \mathrm{H}), 7.19(\mathrm{t}, J=4.8 \mathrm{~Hz}, 1 \mathrm{H}), 4.44(\mathrm{q}, J=10.8 \mathrm{~Hz}, 2 \mathrm{H}) .{ }^{13} \mathrm{C}$ NMR (126 MHz, $\left.\mathrm{CDCl}_{3}\right): \delta 164.2,157.2,137.5,132.7\left(\mathrm{C}-\mathrm{F},{ }^{3} J_{\mathrm{C}-\mathrm{F}}=2.6 \mathrm{~Hz}\right), 130.4,128.4,125.7(\mathrm{C}-\mathrm{F}$, $\left.{ }^{1} J_{\mathrm{C}-\mathrm{F}}=276.9 \mathrm{~Hz}\right), 119.2,40.1\left(\mathrm{C}-\mathrm{F},{ }^{2} J_{\mathrm{C}-\mathrm{F}}=29.8 \mathrm{~Hz}\right) .{ }^{19} \mathrm{~F} \mathrm{NMR}\left(376 \mathrm{MHz}, \mathrm{CDCl}_{3}\right): \delta-65.7(\mathrm{t}, J=$ $10.8 \mathrm{~Hz}, 3 \mathrm{~F}$ ). MS (EI) m/z: 238 [M] $]^{+}, 169$ (100). HRMS (EI-TOF) m/z: [M] Calcd. for $\mathrm{C}_{12} \mathrm{H}_{9} \mathrm{~F}_{3} \mathrm{~N}_{2}$ 238.0718; Found 238.0720.

\section{Conversion of compound into $38^{14}$}

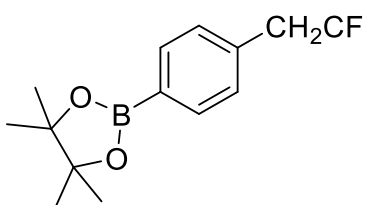

22
(1) $\mathrm{KF}, \mathrm{CH}_{3} \mathrm{CN} / \mathrm{MeOH} / \mathrm{H}_{2} \mathrm{O}$
$\stackrel{\text { (2) } \mathrm{L}-(+) \text { tartaric acid, THF } / \mathrm{CH}_{3} \mathrm{CN}}{\text { (3) } \mathrm{TMSCl}, \mathrm{H}_{2} \mathrm{O} / \mathrm{CH}_{3} \mathrm{CN}} \underset{\mathrm{CF}_{3} \mathrm{H}_{2} \mathrm{C}}{\longrightarrow}$

$38,88 \%$

Step 1: To a $100 \mathrm{~mL}$ round flask were added compound 22 (770 mg, $2.69 \mathrm{mmol}, 1.0$ equiv), methanol $(5 \mathrm{~mL})$, acetonitrile $(5 \mathrm{~mL})$, and $\mathrm{KF}\left(625 \mathrm{mg}, 10.76 \mathrm{mmol}, 4.0\right.$ equiv) in $\mathrm{H}_{2} \mathrm{O}(1.1 \mathrm{~mL})$. After compound 22 was completely dissolved in the solution, a solution of L-(+) tartaric acid (827 $\mathrm{mg}, 5.51 \mathrm{mmol}, 2.05$ equiv) in THF (4 mL) was added dropwise over 1 minute. A white precipitate was formed instantly. After stirring for another $5 \mathrm{~min}$, the reaction mixture was diluted with 
acetonitrile $(8 \mathrm{~mL})$ and filtered. The solid was rinsed with portions of acetonitrile $(3 \mathrm{x} 5 \mathrm{~mL})$, the combined filtrates were concentrated to give the corresponding potassium organotrifluoroborate as a solid.

Step 2: To a $50 \mathrm{~mL}$ round bottle was added the resulting potassium organotrifluoroborate. Acetonitrile (30 mL), TMSCl (232 uL, $2.69 \mathrm{mmol}, 1.0$ equiv) and $\mathrm{H}_{2} \mathrm{O}$ (48 uL, $2.69 \mathrm{mmol}, 1.0$ equiv) were then added. The resulting suspension was stirred for $1 \mathrm{~h}$. The reaction mixture was quenched with saturated aqueous $\mathrm{NaHCO}_{3}(1.2 \mathrm{~mL})$ and dried over $\mathrm{Na}_{2} \mathrm{SO}_{4}$. The mixture was filtered and the filtrate was concentrated to afford arylboronic acid $\mathbf{3 8}(480 \mathrm{mg}, 88 \%)$ as a white solid, which was used for the next step directly without purification.

\section{Synthesis of compound $40^{15}$}

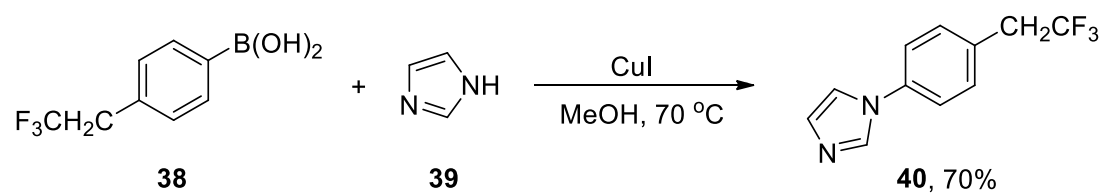

To a $100 \mathrm{~mL}$ three-neck round flask equipped with a condenser were added arylboronic acid 38 (149 mg, $0.73 \mathrm{mmol}, 1.3$ equiv), imidazole 39 (38 mg, $0.56 \mathrm{mmol}, 1.0$ equiv), CuI (16 mg, $0.08 \mathrm{mmol}$, 0.15 equiv) and methanol $(20 \mathrm{~mL})$ under air. After refluxing for $10 \mathrm{~h}$ with continuous pumping of oxygen gas, the mixture was cooled to room temperature and evaporated under reduced pressure. The residue was purified by silica gel column chromatography (petroleum ether: ethyl acetate $=1: 1$ ) to afford product $40(89 \mathrm{mg}, 70 \%)$ as a brown solid (m.p. $\left.41-43{ }^{\circ} \mathrm{C}\right) .{ }^{1} \mathrm{H}$ NMR $\left(400 \mathrm{MHz}, \mathrm{CDCl}_{3}\right): \delta$ $7.87(\mathrm{~s}, 1 \mathrm{H}), 7.430-7.38(\mathrm{~m}, 4 \mathrm{H}), 7.29(\mathrm{~s}, 1 \mathrm{H}), 7.23(\mathrm{~s}, 1 \mathrm{H}), 3.42$ (q, $J=10.8 \mathrm{~Hz}, 2 \mathrm{H}) .{ }^{13} \mathrm{C}$ NMR $(126$ $\left.\mathrm{MHz}_{\mathrm{CDCl}}\right): \delta 131.6,129.4\left(\mathrm{C}-\mathrm{F},{ }^{3} J_{\mathrm{C}-\mathrm{F}}=3.0 \mathrm{~Hz}\right), 125.5\left(\mathrm{C}-\mathrm{F},{ }^{1} J_{\mathrm{C}-\mathrm{F}}=277.4 \mathrm{~Hz}\right), 121.5,39.6(\mathrm{C}-\mathrm{F}$, $\left.{ }^{2} J_{\mathrm{C}-\mathrm{F}}=30.0 \mathrm{~Hz}\right) .{ }^{19} \mathrm{~F} \mathrm{NMR}\left(376 \mathrm{MHz}, \mathrm{CDCl}_{3}\right): \delta-66.0(\mathrm{t}, J=10.9 \mathrm{~Hz}, 3 \mathrm{~F}) . \mathrm{MS}(\mathrm{EI}) \mathrm{m} / \mathrm{z}: 226[\mathrm{M}]^{+}$, 226 (100). HRMS (EI-TOF) m/z: [M] ${ }^{+}$Calcd. for $\mathrm{C}_{11} \mathrm{H}_{9} \mathrm{~F}_{3} \mathrm{~N}_{2} 226.0718$; Found 226.0710. 


\section{Radical Trapping Experiments}

\section{Radical inhibition experiments ${ }^{a}$}

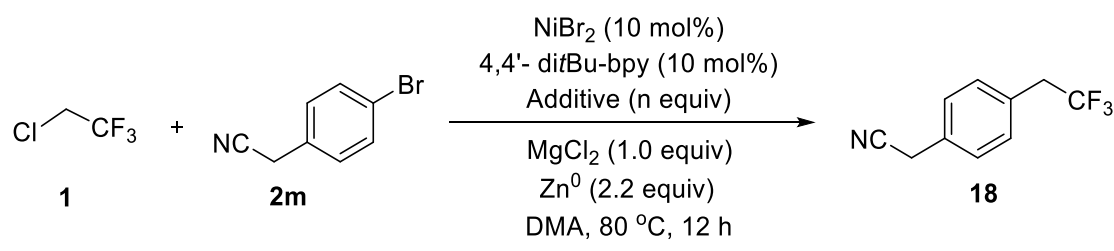

\begin{tabular}{ccc}
\hline Entry & Additive (n equiv) & $\mathbf{1 8}$, Yield $(\%)^{b}$ \\
\hline 1 & none & $78 \%,(82 \%)^{c}$ \\
2 & $1,4-$ Dinitrobenzene (0.2 equiv) & $0 \%$ \\
3 & Hydroquinone (1.5 equiv) & $23 \%$ \\
\hline
\end{tabular}

${ }^{a}$ Reaction conditions (unless otherwise specified): 1 ( $0.4 \mathrm{mmol}, 1.0$ equiv), aryl bromide (1.5 equiv), DMA (4 mL). ${ }^{b}$ Determined by ${ }^{19} \mathrm{~F}$ NMR using fluorobenzene as an internal standard. ${ }^{c}$ Isolated yield.

Procedure: To a $25 \mathrm{~mL}$ of Schlenk tube were added arylbromide $\mathbf{2 m}$ (58.8 $\mathrm{mg}, 0.3 \mathrm{mmol}$, 1.5 equiv), zinc powder ( $28.8 \mathrm{mg}, 0.44 \mathrm{mmol}, 2.2$ equiv), 4,4'-dibutylbipyridine (5.4 mg, $0.02 \mathrm{mmol}, 0.1$ equiv) $\mathrm{MgCl}_{2}$ (19.1 mg, $0.2 \mathrm{mmol}, 1.0$ equiv), and the corresponding additive. The tube was then evacuated and back-filled with argon for three times. $\mathrm{NiBr}_{2}(4.4 \mathrm{mg}, 0.2 \mathrm{mmol}, 0.1$ equiv), anhydrous solution of 1 in DMA (0.2 mmol, 1.0 equiv) and anhydrous DMA (2 mL) were added under argon. After stirring for $12 \mathrm{~h}$ at $80{ }^{\circ} \mathrm{C}$, the reaction was cooled to room temperature and quenched by water $(2 \mathrm{~mL})$ and ethyl acetate $(2 \mathrm{~mL})$. The yield was determined by ${ }^{19} \mathrm{~F}$ NMR using fluorobenzene as an internal standard.

\section{Radical-clock experiment}
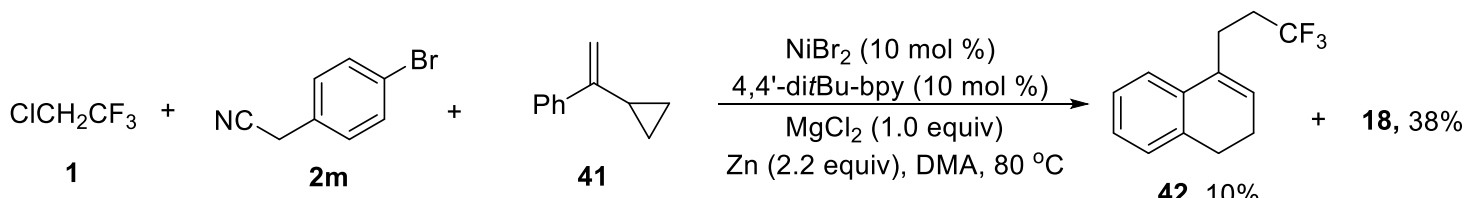

Procedure: To a $25 \mathrm{~mL}$ of Schlenk tube were added arylbromide $\mathbf{2 m}(117.6 \mathrm{mg}, 0.6 \mathrm{mmol}, 1.5$ equiv), zinc powder (57.5 mg, $0.88 \mathrm{mmol}, 2.2$ equiv), 4,4'-dibutylbipyridine (10.7 $\mathrm{mg}, 0.04 \mathrm{mmol}$, 0.1 equiv) and $\mathrm{MgCl}_{2}$ (38.1 $\mathrm{mg}, 0.4 \mathrm{mmol}, 1.0$ equiv). The tube was then evacuated and refilled with argon for three times. $\mathrm{NiBr}_{2}(8.7 \mathrm{mg}, 0.4 \mathrm{mmol}, 0.1$ equiv), anhydrous solution of 1 ( $0.4 \mathrm{mmol}, 1.0$ equiv) in DMA, (1-cyclopropylvinyl)benzene 41 (86.5 mg, $0.6 \mathrm{mmol}, 1.5$ equiv) and anhydrous DMA (4 mL) were added under argon. After stirring at $80{ }^{\circ} \mathrm{C}$ for $12 \mathrm{~h}$, the reaction mixture was 
cooled to room temperature and quenched by water $(2 \mathrm{~mL})$ and ethyl acetate $(2 \mathrm{~mL})$. The resulting mixture was filtered and extracted with ethyl acetate $(5 \mathrm{~mL} \times 3)$. The combined organic layers were concentrated, and the residue was purified by flash chromatography on silica gel (petroleum $300 \mathrm{~mL}$, then petroleum ether : ethyl acetate $=8: 1)$ to give compound 42 and $\mathbf{1 8}$. Compound 42 (9 mg, 10\% yield) was a light yellow oil. ${ }^{1} \mathrm{H}$ NMR (400 MHz, $\left.\mathrm{CDCl}_{3}\right): \delta$ 7.24-7.20 (m, 2H), 7.19-7.15 (m, $\left.2 \mathrm{H}\right)$, $5.93(\mathrm{t}, J=4.6 \mathrm{~Hz}, 1 \mathrm{H}), 2.78-2.68(\mathrm{~m}, 4 \mathrm{H}), 2.39-2.24(\mathrm{~m}, 4 \mathrm{H}) .{ }^{13} \mathrm{C} \mathrm{NMR}\left(100 \mathrm{MHz}, \mathrm{CDCl}_{3}\right): \delta 136.8$, 133.8, 128.6, 127.8, 127.1 (C-F, $\left.{ }^{1} J_{\mathrm{C}-\mathrm{F}}=276.7 \mathrm{~Hz}\right), 127.0,126.5,126.1,122.1,33.1\left(\mathrm{C}-\mathrm{F},{ }^{2} J_{\mathrm{C}-\mathrm{F}}=28.2\right.$ $\mathrm{Hz}), 28.2,25.2\left(\mathrm{C}-\mathrm{F},{ }^{3} J_{\mathrm{C}-\mathrm{F}}=3.2 \mathrm{~Hz}\right), 23.0 .{ }^{19} \mathrm{~F} \mathrm{NMR}\left(376 \mathrm{MHz}, \mathrm{CDCl}_{3}\right): \delta-66.6(\mathrm{t}, J=10.8 \mathrm{~Hz}, 3 \mathrm{~F})$. MS (EI) m/z: 226 [M] $]^{+}, 129$ (100). HRMS (EI-TOF) m/z: [M] Calcd. for $\mathrm{C}_{13} \mathrm{H}_{13} \mathrm{~F}_{3}$ 226.0969; Found 226.0971. 


\section{References}

(1) Svejstrup, T. D.; Ruffoni, A.; Juliá, F.; Aubert, V. M.; Leonori, D. Synthesis of arylamines via aminium radicals. Angew. Chem. Int. Ed. 2017, 56, 14948-14952.

(2) Guo, S.; AbuSalim, D. I.; Cook, S. P. Aqueous benzylic C-H trifluoromethylation for late-stage functionalization. J. Am. Chem. Soc. 2018, 140, 12378-12382.

(3) Yang, Y.; Zhou, Q.; Cai, J.; Xue, T.; Liu, Y.; Jiang, Y.; Y. Su, Chung, L. W.; Vicic, D. A. Exploiting the trifluoroethyl group as a precatalyst ligand in nickel-catalyzed Suzuki-type alkylations. Chem. Sci. 2019, 10, 5275-5282.

(4) Zhao, Y.; Hu, J. Palladium-catalyzed 2,2,2-trifluoroethylation of organoboronic acids and esters. Angew. Chem. Int. Ed. 2012, 51, 1033-1036.

(5) Liang, A.; Li, X.; Liu, D.; Li, J.; Zou, D.; Wu, Y.; Wu, Y. The palladium-catalyzed cross-coupling reactions of trifluoroethyl iodide with aryl and heteroaryl boronic acid esters. Chem. Commun. 2012, $48,8273-8275$

(6) Qiao, Y.; Si, T.; Yang, M.-H.; Altman, R. A. Metal-free trifluoromethylation of aromatic and heteroaromatic aldehydes and ketones. J. Org. Chem. 2014, 79, 7122-7131.

(7) Paeth, M.; Carson, W.; Luo, J.-H.; Tierney, D.; Cao, Z.; Cheng, M.-J.; Liu, W. Copper-mediated trifluoromethylation of benzylic Csp ${ }^{3}-\mathrm{H}$ bonds. Chem. Eur. J. 2018, 24, 11559-11563.

(8) Kautzky, J. A.; Wang, T.; Evans, R. W.; MacMillan, D. W. C. Decarboxylative Trifluoromethylation of Aliphatic Carboxylic Acids. J. Am. Chem. Soc. 2018, 140, 6522-6526.

(9) Xu, S.; Chen, H.-H.; Dai, J.-J.; Xu, H.-J. Copper-promoted reductive coupling of aryl iodides with 1,1,1-trifluoro-2-iodoethane. Org. Lett. 2014, 16, 2306-2309.

(10) Leng, F.; Wang, Y.; Li, H.; Li, J.; Zou, D.; Wu, Y.; Wu, Y. Facile synthesis of trifluoroethyl compounds by the Suzuki cross-coupling reactions of $\mathrm{CF}_{3} \mathrm{CH}_{2} \mathrm{OTs}$ with arylboronic acids. Chem. Commun. 2013, 49, 10697-10699

(11) Kawai, H.; Furukawa, T.; Nomura, Y.; Tokunaga, E.; Shibata, N. Cu-mediated chemoselective trifluoromethylation of benzyl bromides using shelf-stable electrophilic trifluoromethylating reagents. Org. Lett. 2011, 13, 3596-3599.

(12) Li, H.; Sheng, J.; Liao, G.-X.; Wu, B.-B.; Ni, H.-Q.; Li, Y.; Wang, X.-S. Nickel-catalyzed direct trifluoroethylation of aryl Iodides with 1,1,1-trifluoro-2-iodoethane via reductive coupling. $A d v$. Synth. Catal. 2020, 362, doi: org/10.1002/adsc.202000985.

(13) (a) Lee, E.; Hooker, J. M.; Ritter, T. Nickel-Mediated Oxidative Fluorination for PET with Aqueous $\left[{ }^{18} \mathrm{~F}\right]$ Fluoride. J. Am. Chem. Soc. 2012, 134, 17456-17458. (b) Asachenko, A. F.; Sorochkina, K. R.; Dzhevakov, P. B.; Topchiy, M. A.; Nechaev, M. S. Suzuki-miyaura crosscoupling under solvent-free conditions. Adv. Synth. Catal. 2013, 355, 3553-3557.

(14) (a) Lennox, A. J. J.; Lloyd-Jones, G. C. Preparation of Organotrifluoroborate Salts: Precipitation-Driven Equilibrium under Non-Etching Conditions. Angew. Chem. Int. Ed. 2012, 51, 9385-9388. (b) Yuen, A. K. L.; Hutton, C. A. Deprotection of pinacolyl boronate esters via hydrolysis of intermediate potassium trifluoroborates Tetrahedron Lett. 2005, 46, 7899-7903.

(15) Chu, Y.; Deng, H.; Cheng, J.-P. An acidity scale of 1,3-dialkylimidazolium salts in dimethyl sulfoxide solution. J. Org. Chem. 2007, 72, 7790-7793. 
8. ${ }^{1} \mathrm{H},{ }^{19} \mathrm{~F}$ and ${ }^{13} \mathrm{C}$ NMR spectra copies of compounds 3, 7-35, 37, 40, 42

\section{(2, 2, 2-Trifluoroethyl)benzene (3)}
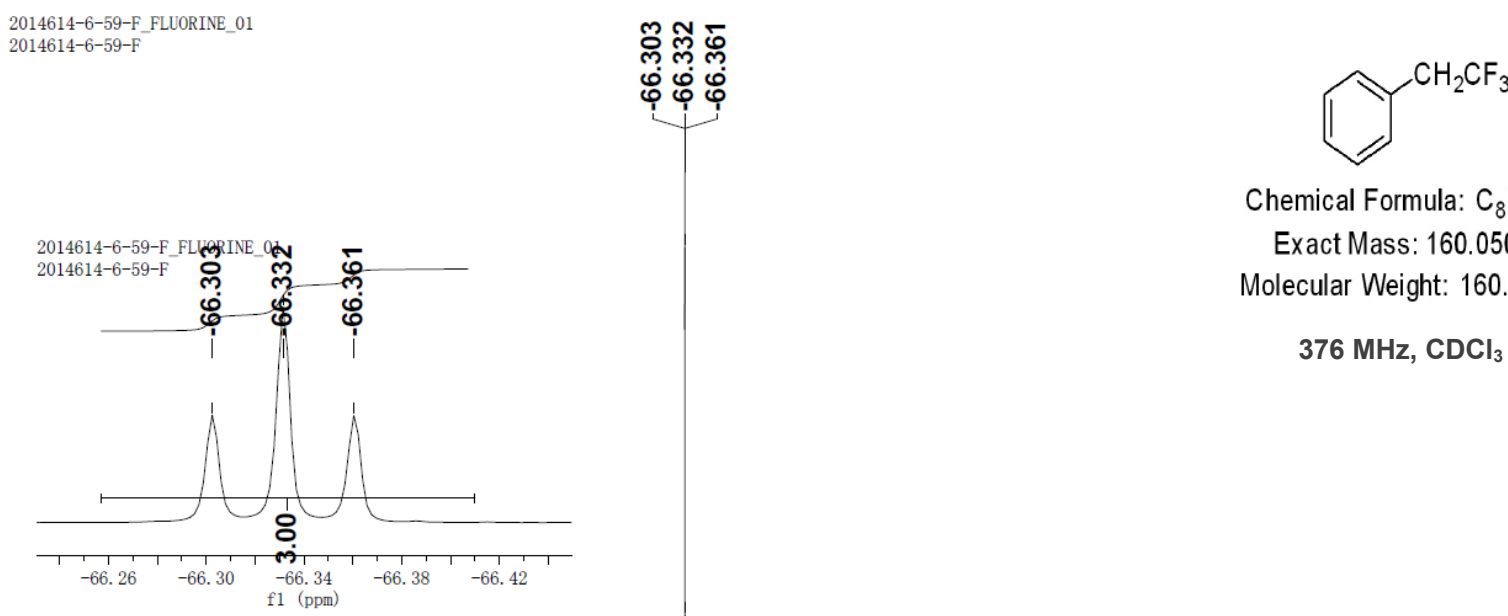

Chemical Formula: $\mathrm{C}_{8} \mathrm{H}_{7} \mathrm{~F}_{3}$

Exact Mass: 160.0500

Molecular Weight: 160.1392

$376 \mathrm{MHz}, \mathrm{CDCl}_{3}$

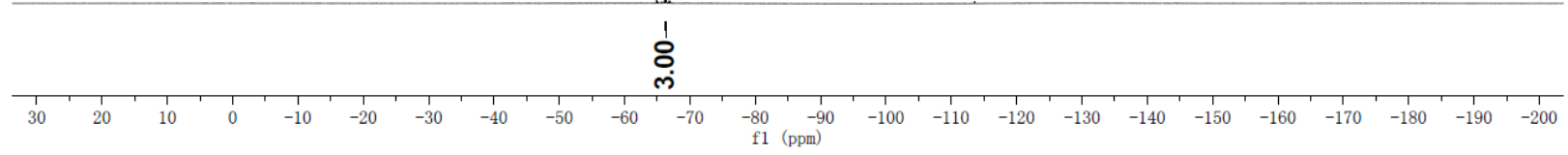

文作 : E: \data $2014614-6-59$. D

裆集：21 Jul $2016 \quad 10: 23$, 使用采集方法 50-280. M

仪器: GCMS

样谷:

其他信息

样品瓶宂: 1

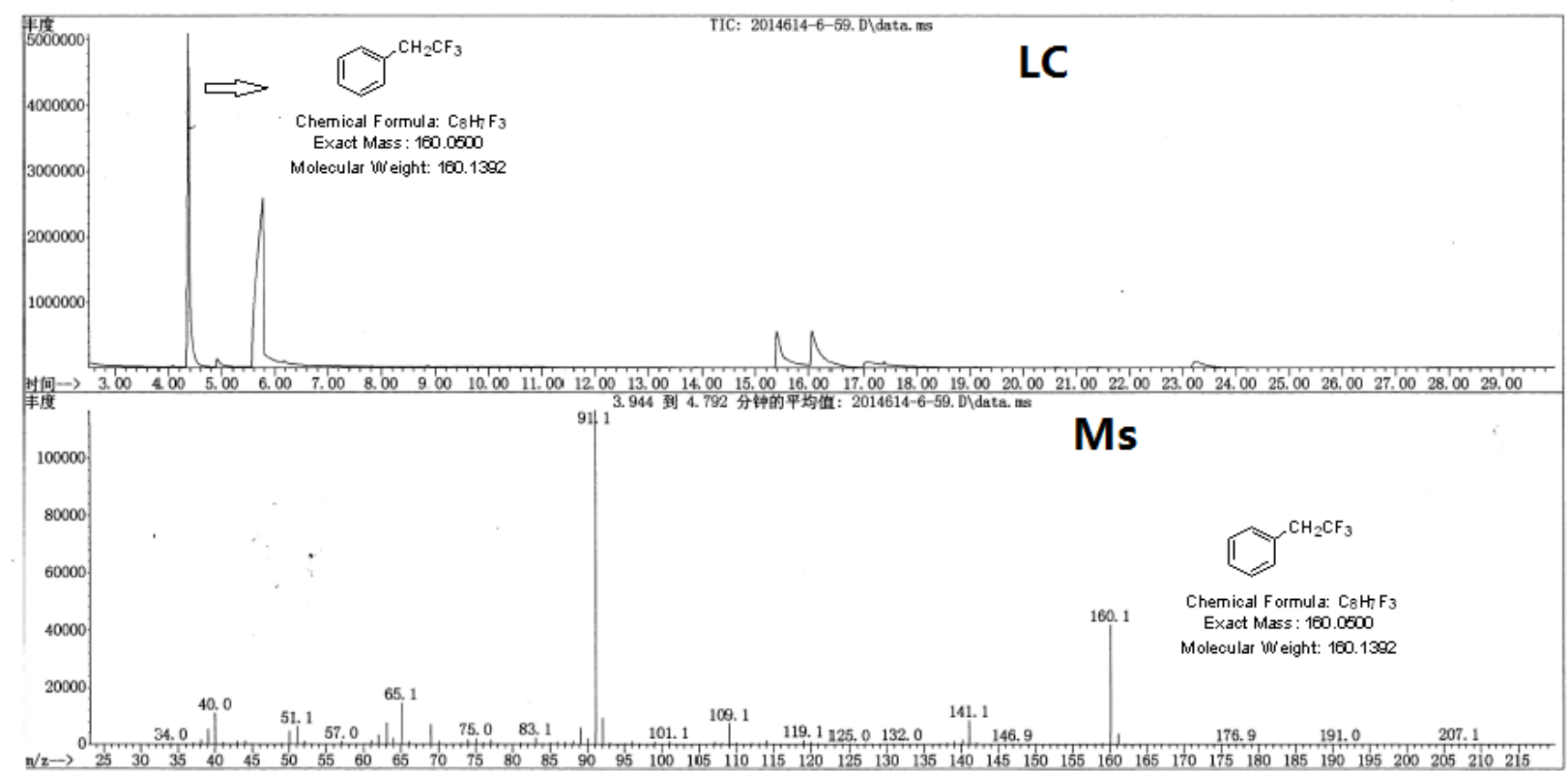


1-(tert-Butyl)-4-(2, 2, 2-trifluoroethyl)benzene (7)
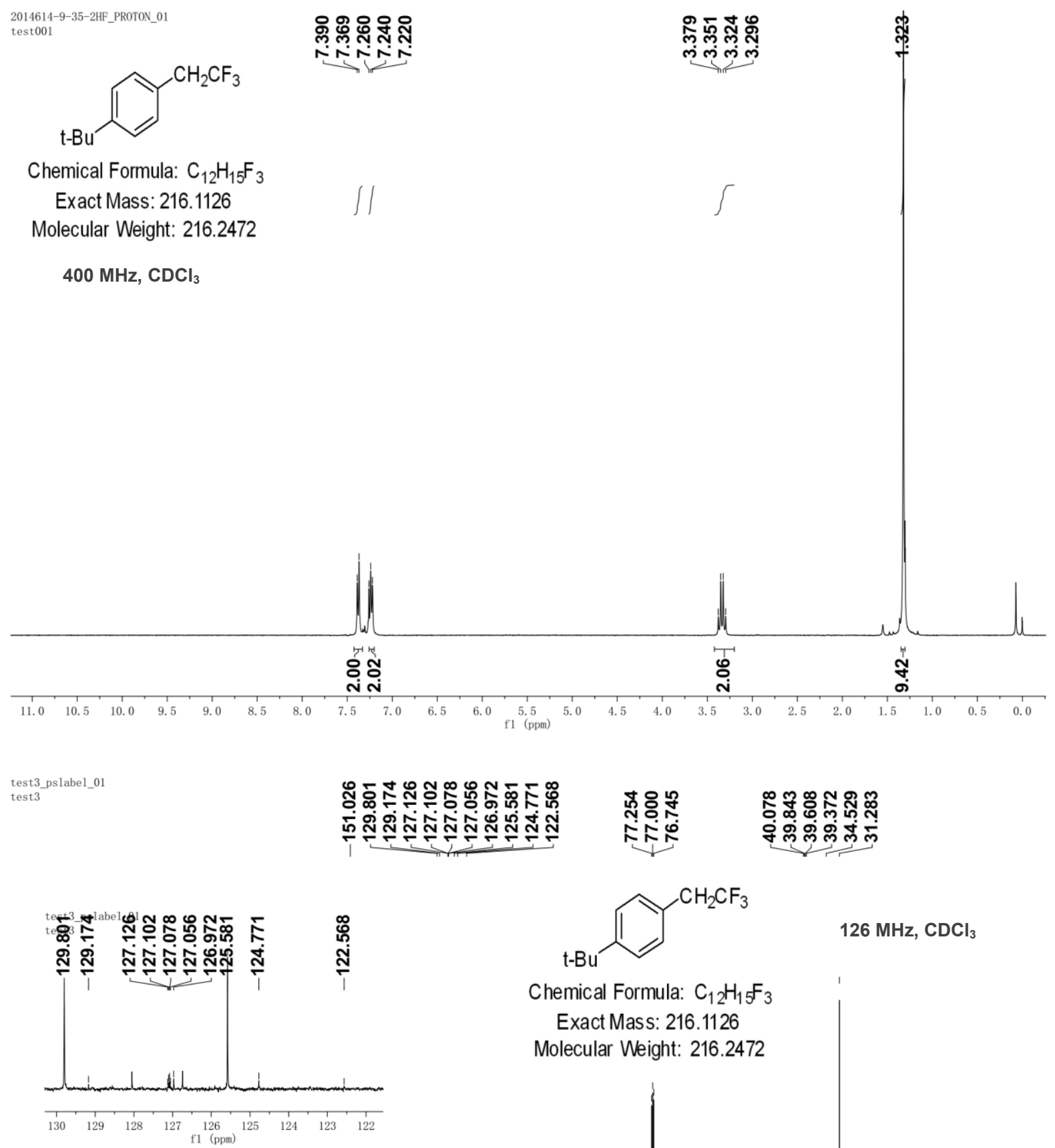
Chemical Formula: $\mathrm{C}_{12} \mathrm{H}_{15} \mathrm{~F}_{3}$

Exact Mass: 216.1126 Molecular Weight: 216.2472

$126 \mathrm{MHz}, \mathrm{CDCl}_{3}$

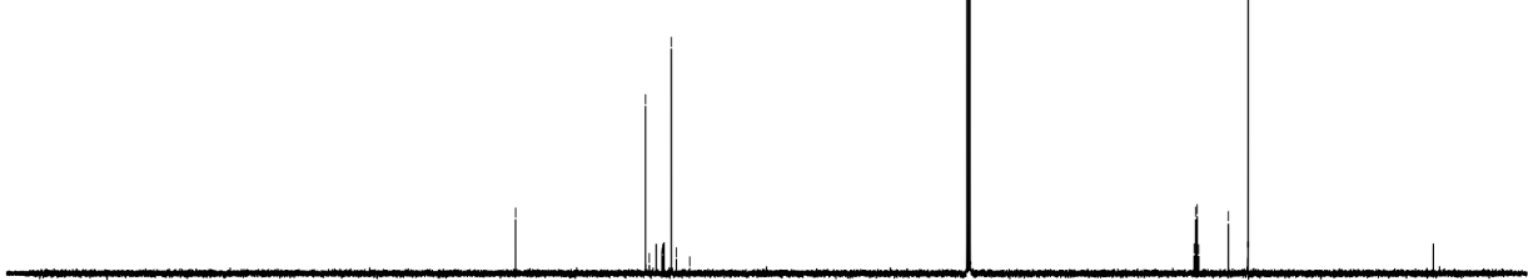

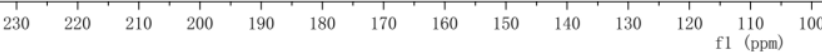




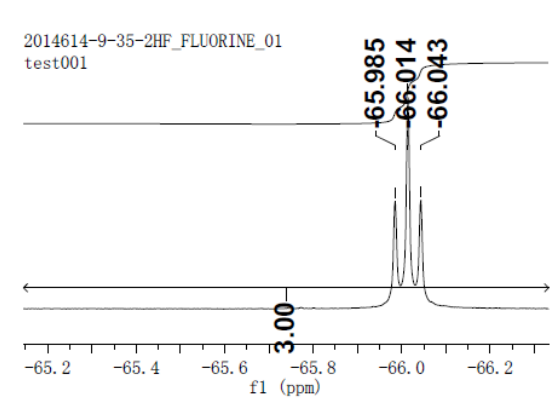

$\mathrm{t}-\mathrm{Bu}$<smiles>CC(C)(C)c1ccc(CC(F)(F)F)cc1</smiles>

Chemical Formula: $\mathrm{C}_{12} \mathrm{H}_{15} \mathrm{~F}_{3}$

Exact Mass: 216.1126

Molecular Weight: 216.2472

$376 \mathrm{MHz}, \mathrm{CDCl}_{3}$

\section{'T:}

$\begin{array}{cc}-80 & -90 \\ \mathrm{f} 1 & (\mathrm{ppm})\end{array}$

1-(tert-Butyl)-3-(2, 2, 2-trifluoroethyl)benzene (8)

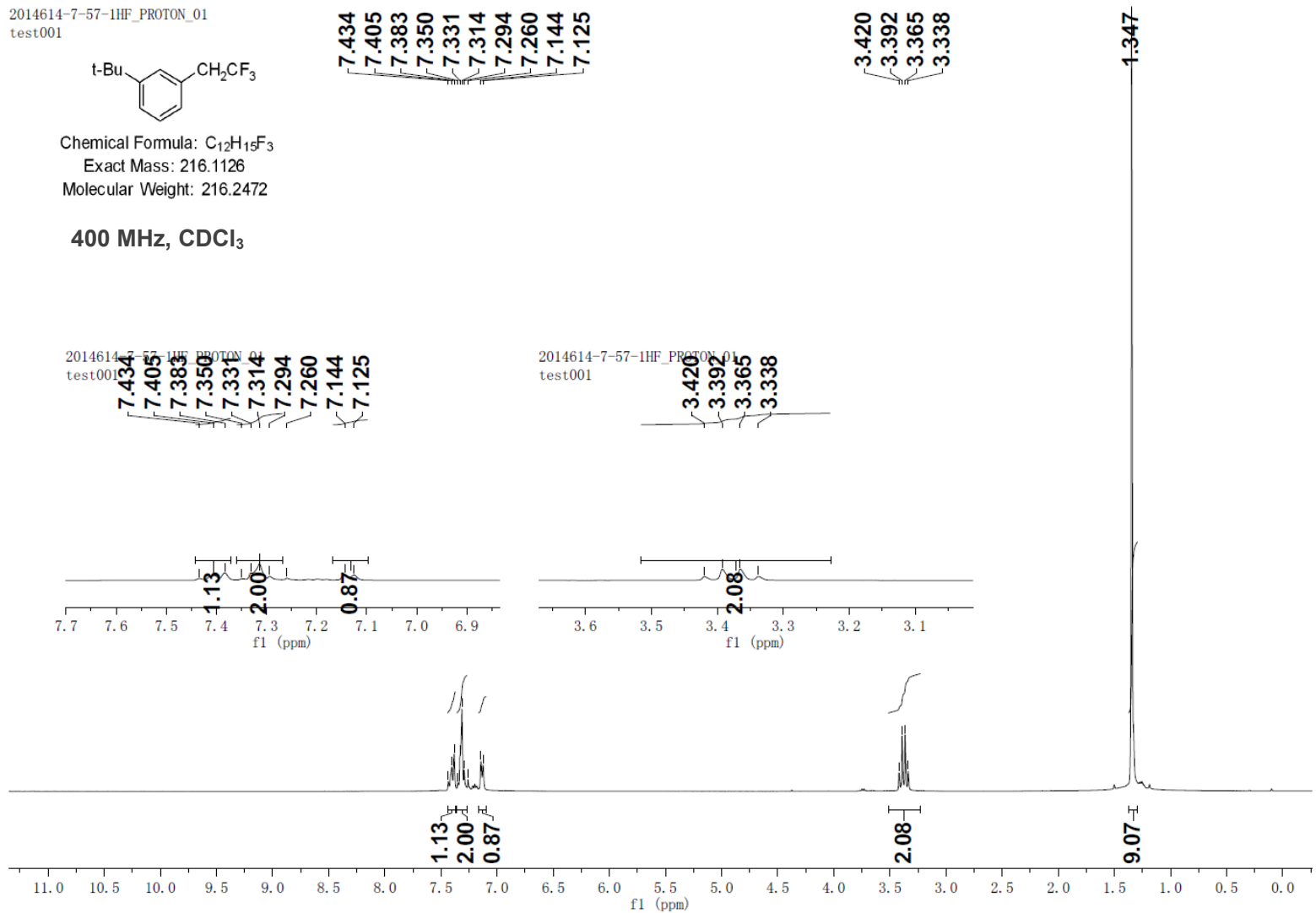



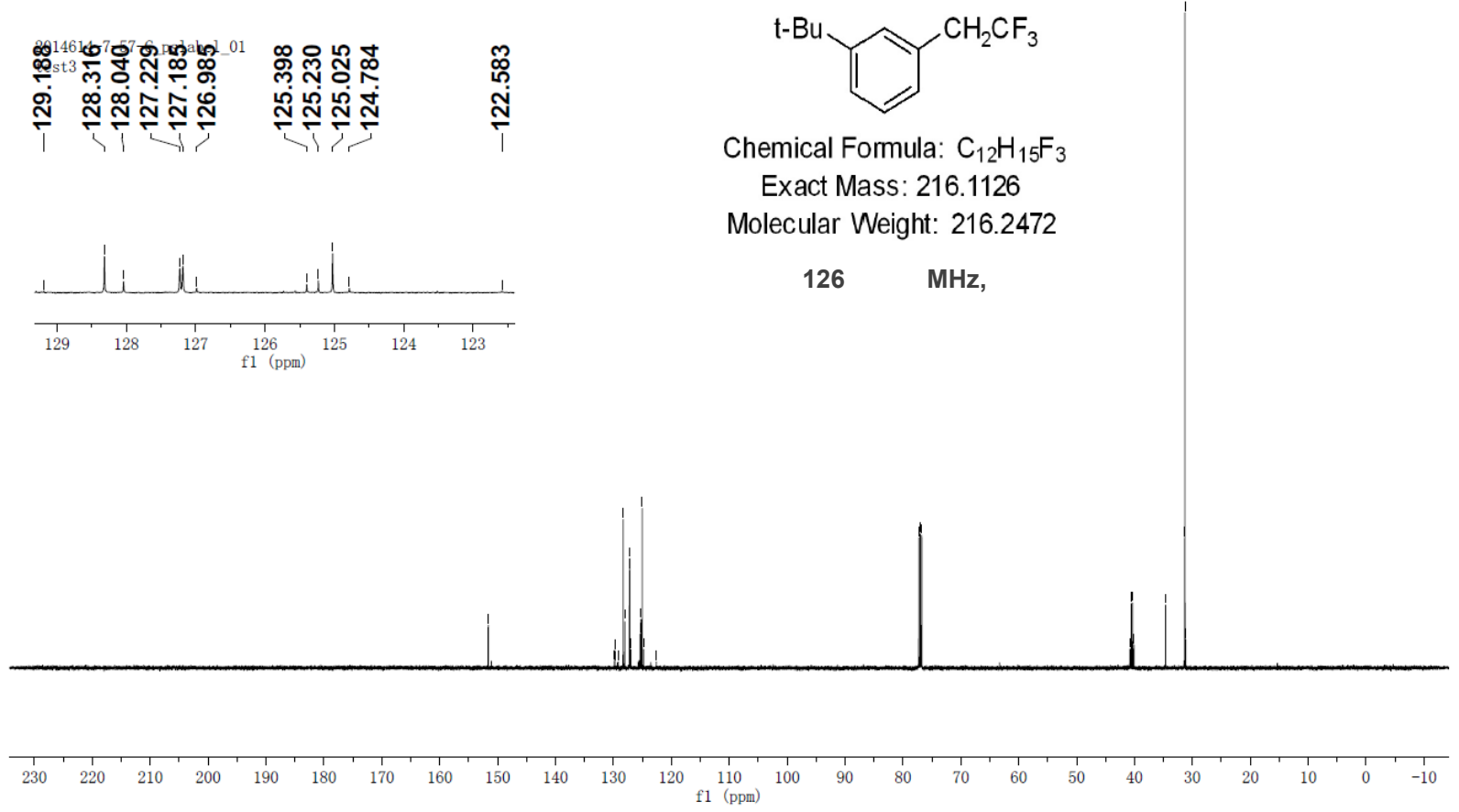

2014614-7-57-1HF_FLUORINE_01

test001

호ำ

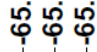
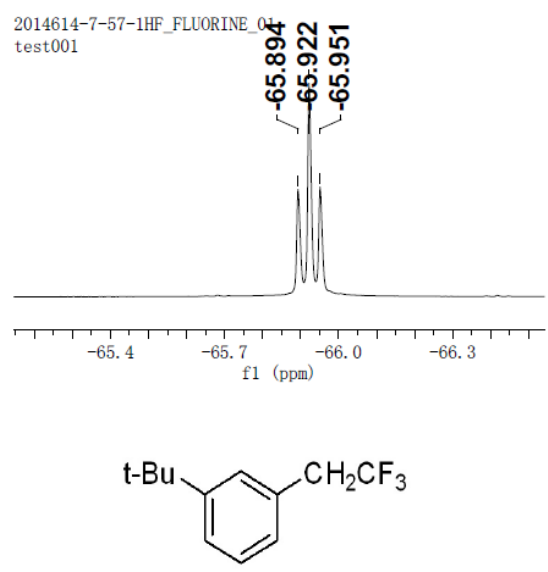

Chemical Formula: $\mathrm{C}_{12} \mathrm{H}_{15} \mathrm{~F}_{3}$

Exact Mass: 216.1126

Molecular Weight: 216.2472

$376 \mathrm{MHz}, \mathrm{CDCl}_{3}$ 

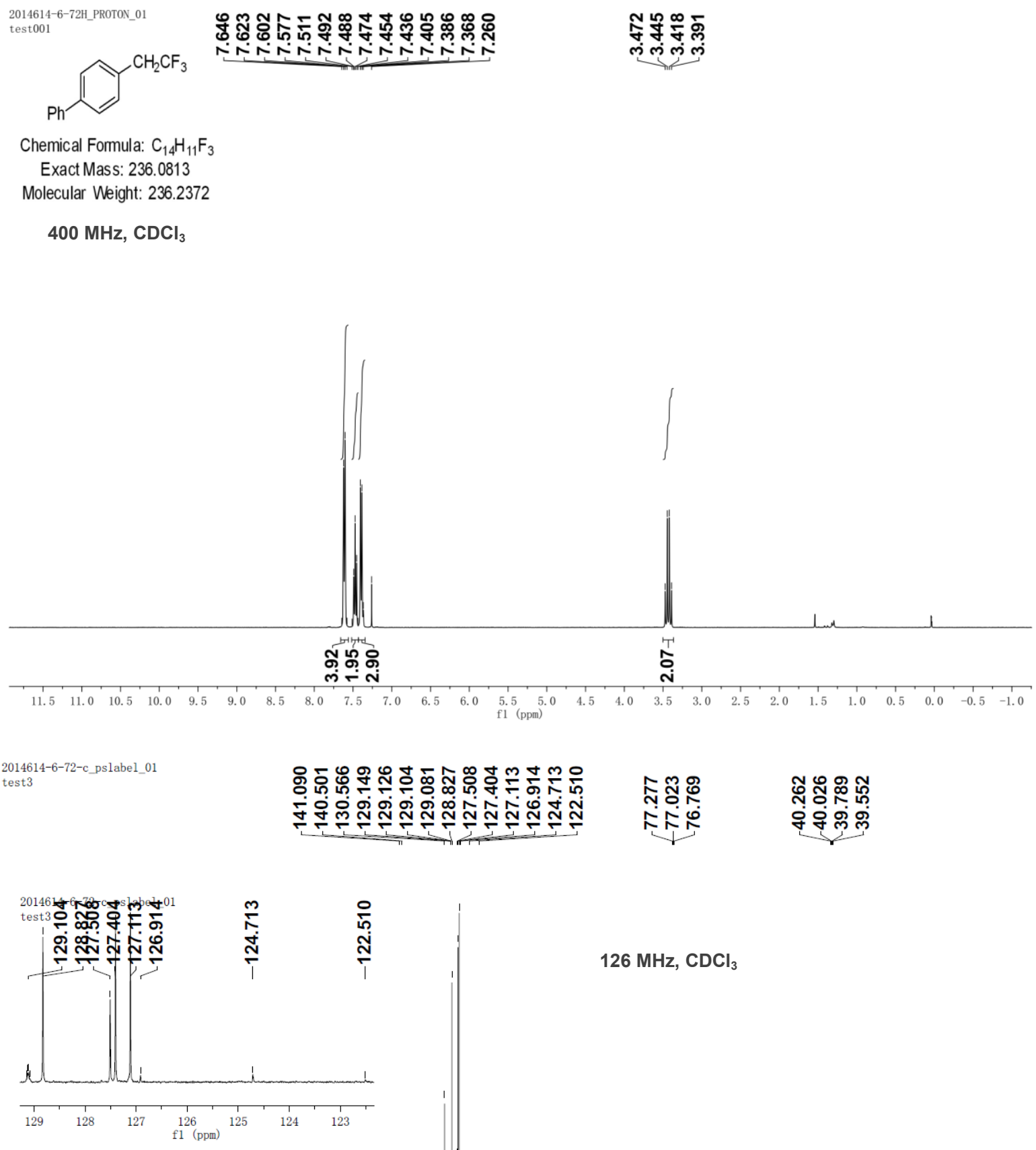

$126 \mathrm{MHz}^{\mathrm{CDCl}_{3}}$<smiles>FC(F)(F)Cc1ccc(-c2ccccc2)cc1</smiles>

Chemical Formula: $\mathrm{C}_{14} \mathrm{H}_{11} \mathrm{~F}_{3}$

Exact Mass: 236.0813 Molecular Weight: 236.2372 


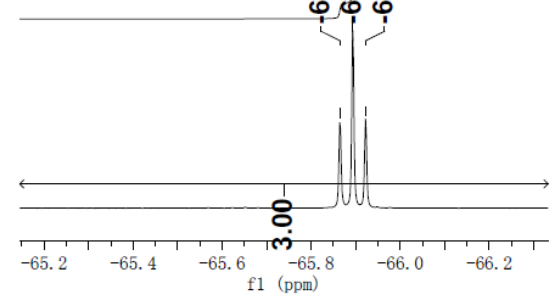

$376 \mathrm{MHz}, \mathrm{CDCl}_{3}$

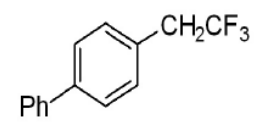

Chemical Formula: $\mathrm{C}_{14} \mathrm{H}_{11} \mathrm{~F}_{3}$

Exact Mass: 236.0813

Molecular Weight: 236.2372

\section{㻤}

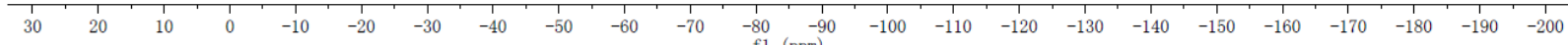

\section{1-Methoxy-3-(2, 2, 2-trifluoroethyl)benzene (10)}

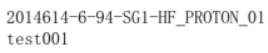

Chemical Formula: $\mathrm{C}_{9} \mathrm{H}_{9} \mathrm{~F}_{3} \mathrm{O}$

Exact Mass: 190.0605

Molecular Weight: 190.1652

$400 \mathrm{MHz}, \mathrm{CDCl}_{3}$

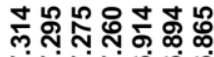

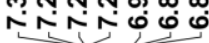

:

लं लंखिं

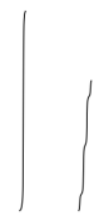

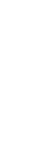

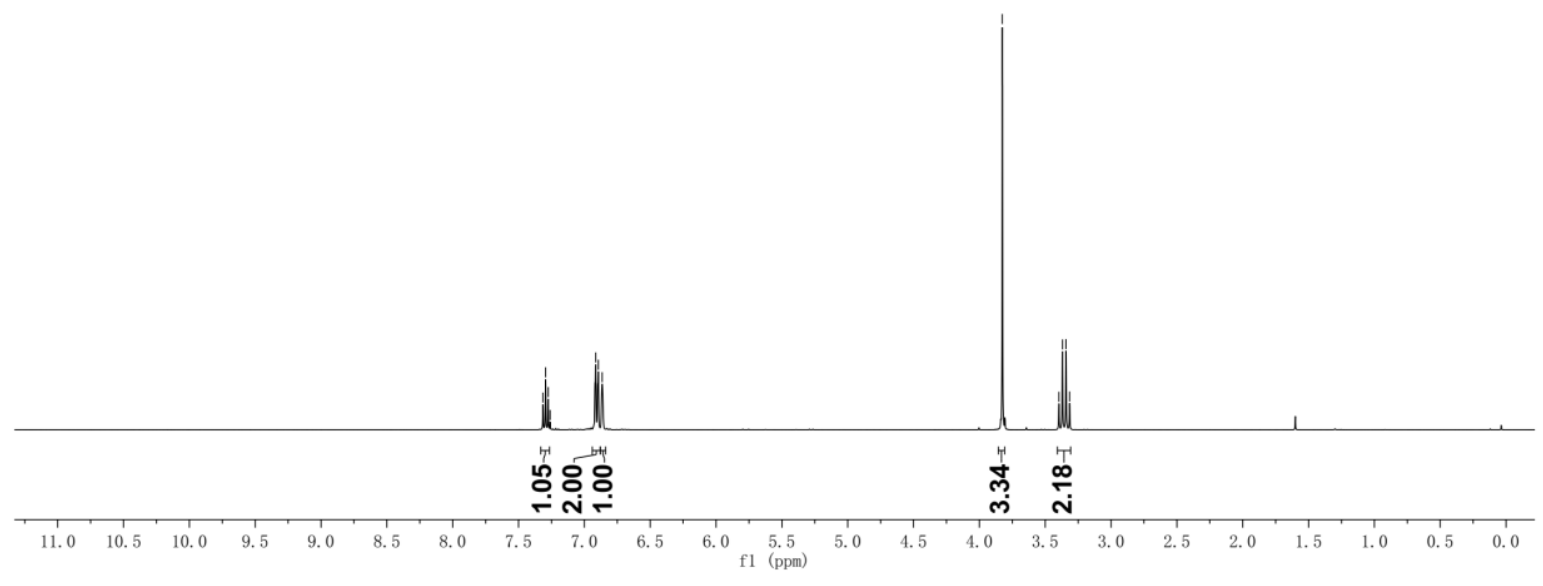



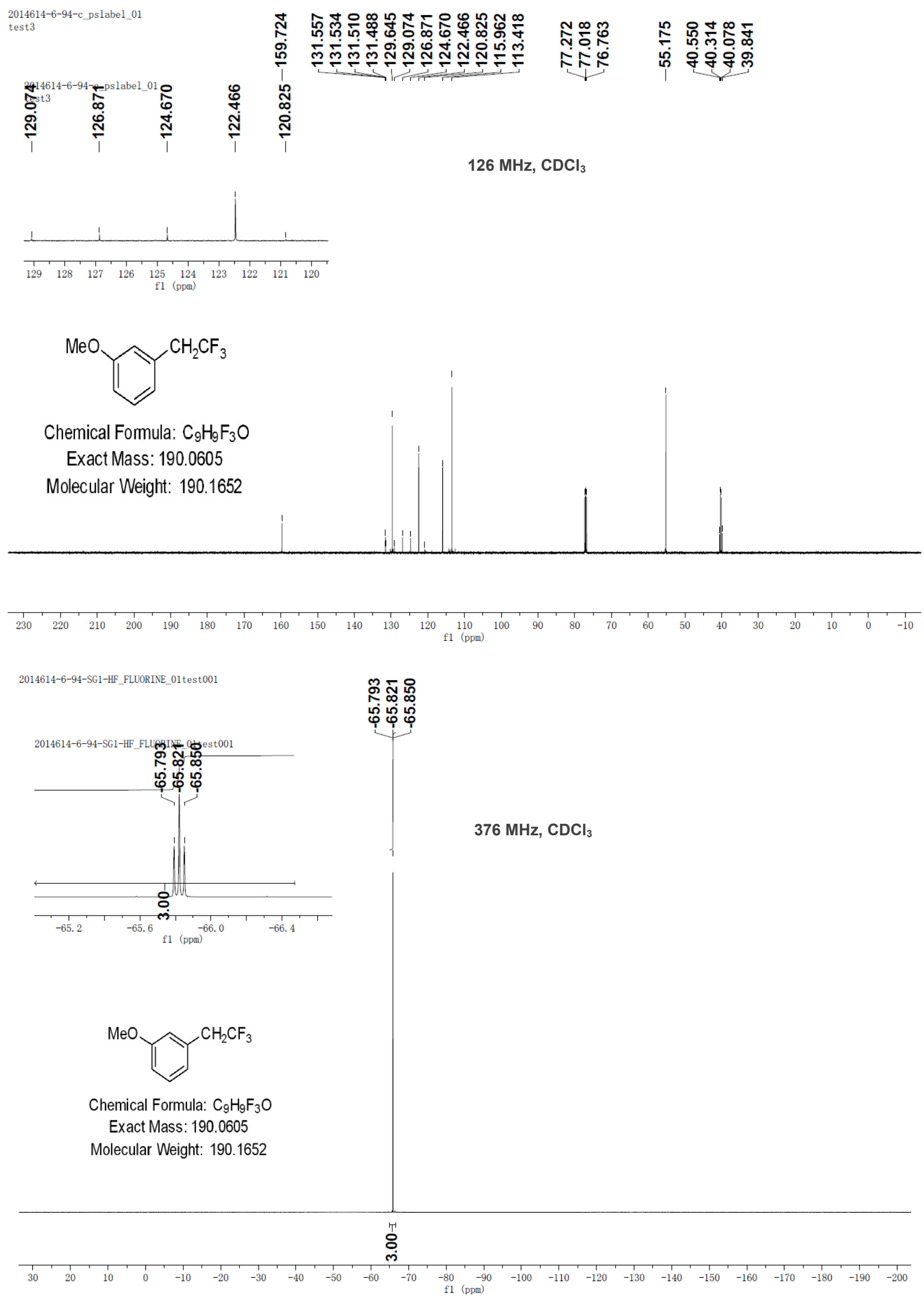
1-(Benzyloxy)-3-(2, 2, 2-trifluoroethyl)benzene (11)

2014614-7-4-H_PROTON_01

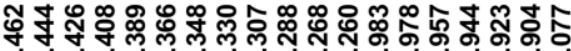

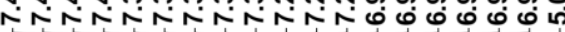

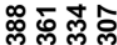

BnO $\mathrm{CH}_{2} \mathrm{CF}_{3}$

Chemical Formula: $\mathrm{C}_{15} \mathrm{H}_{13} \mathrm{~F}_{3} \mathrm{O}$

Exact Mass: 266.0918

Molecular Weight: 266.2632

$400 \mathrm{MHz}, \mathrm{CDCl}_{3}$
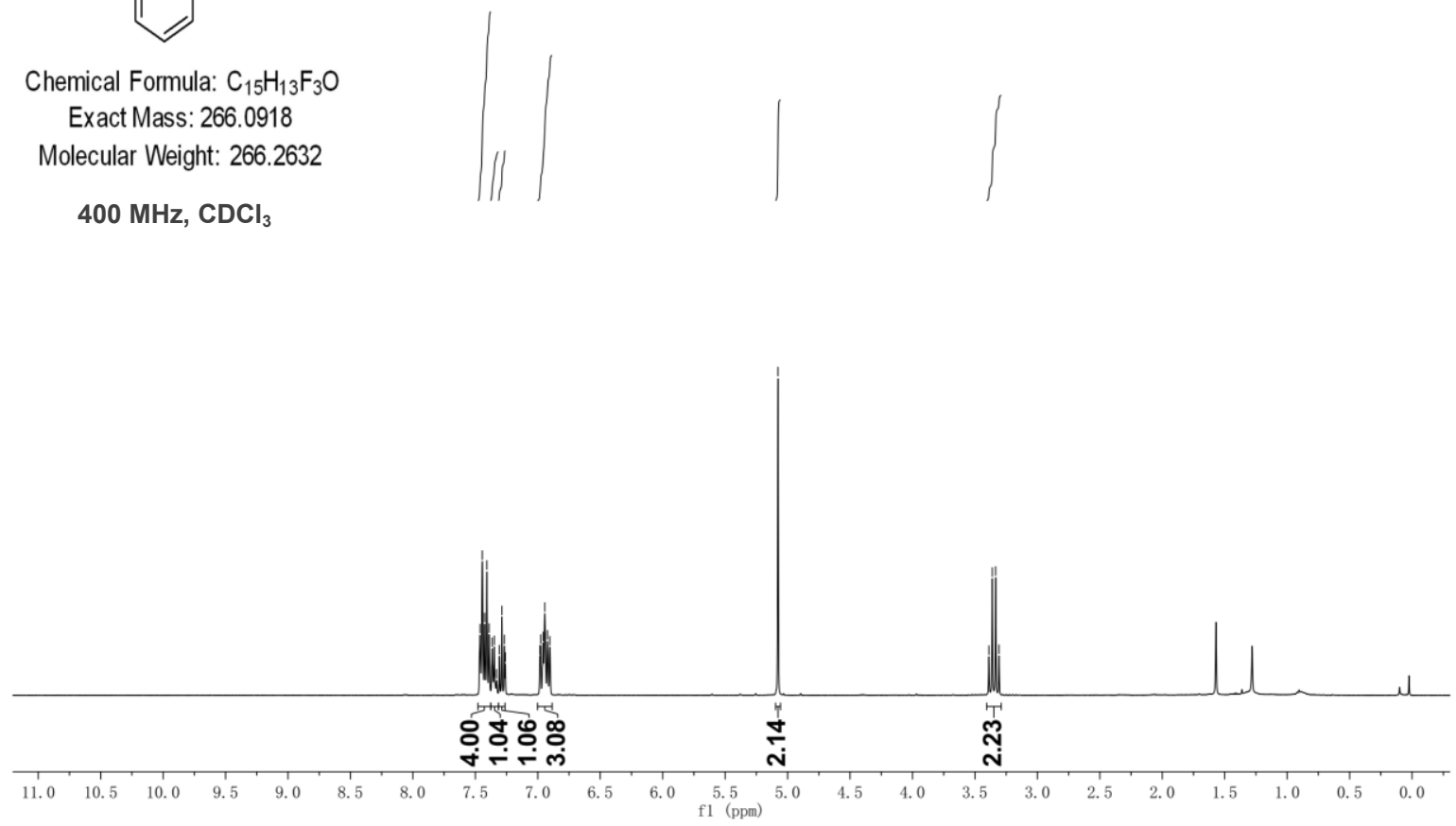

2014614-7-4-C_pslabel_01

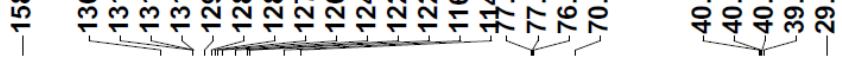

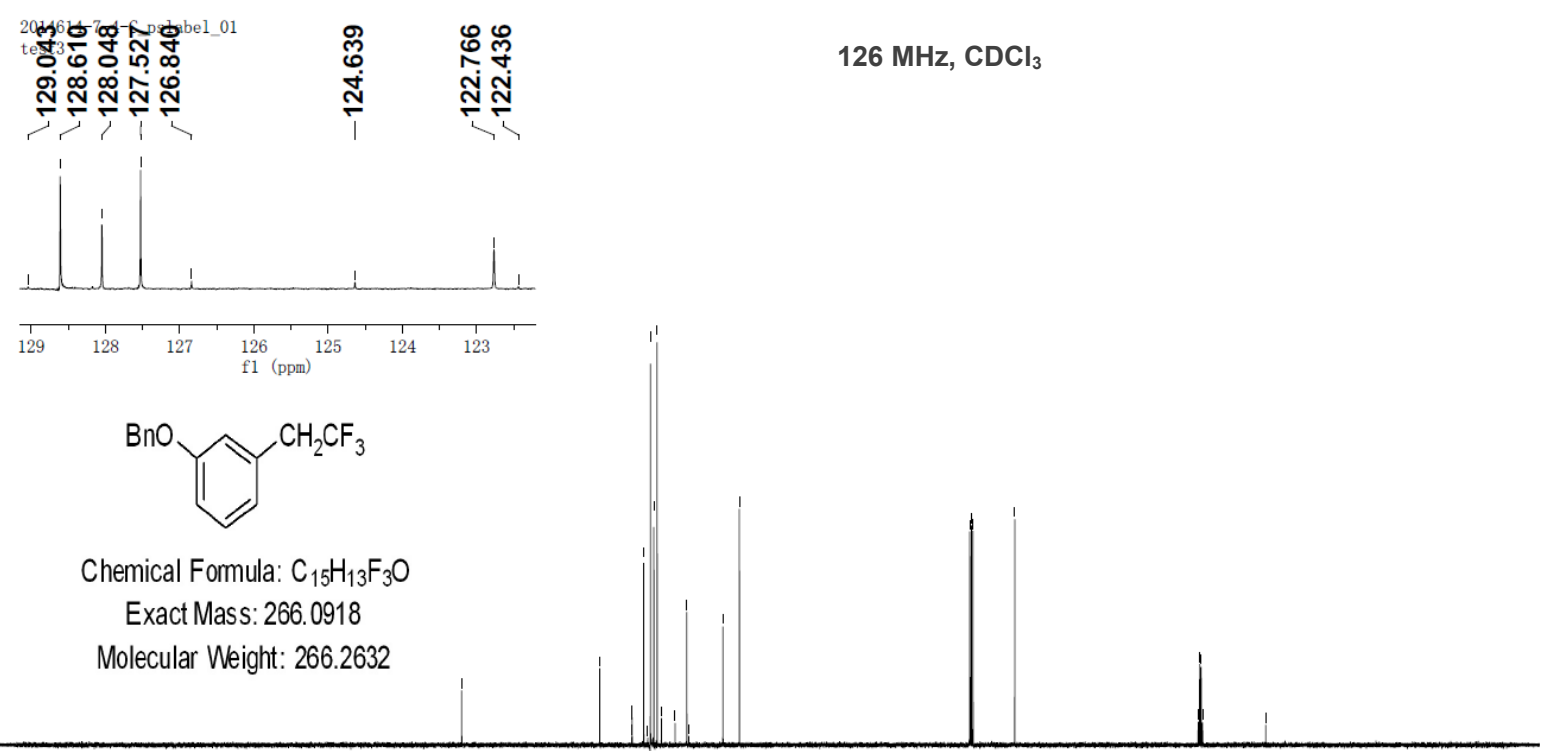

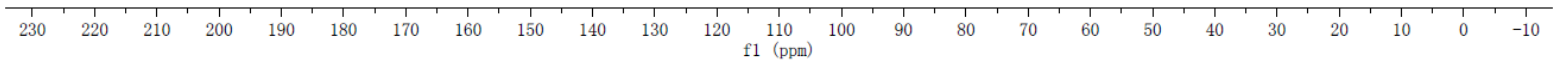




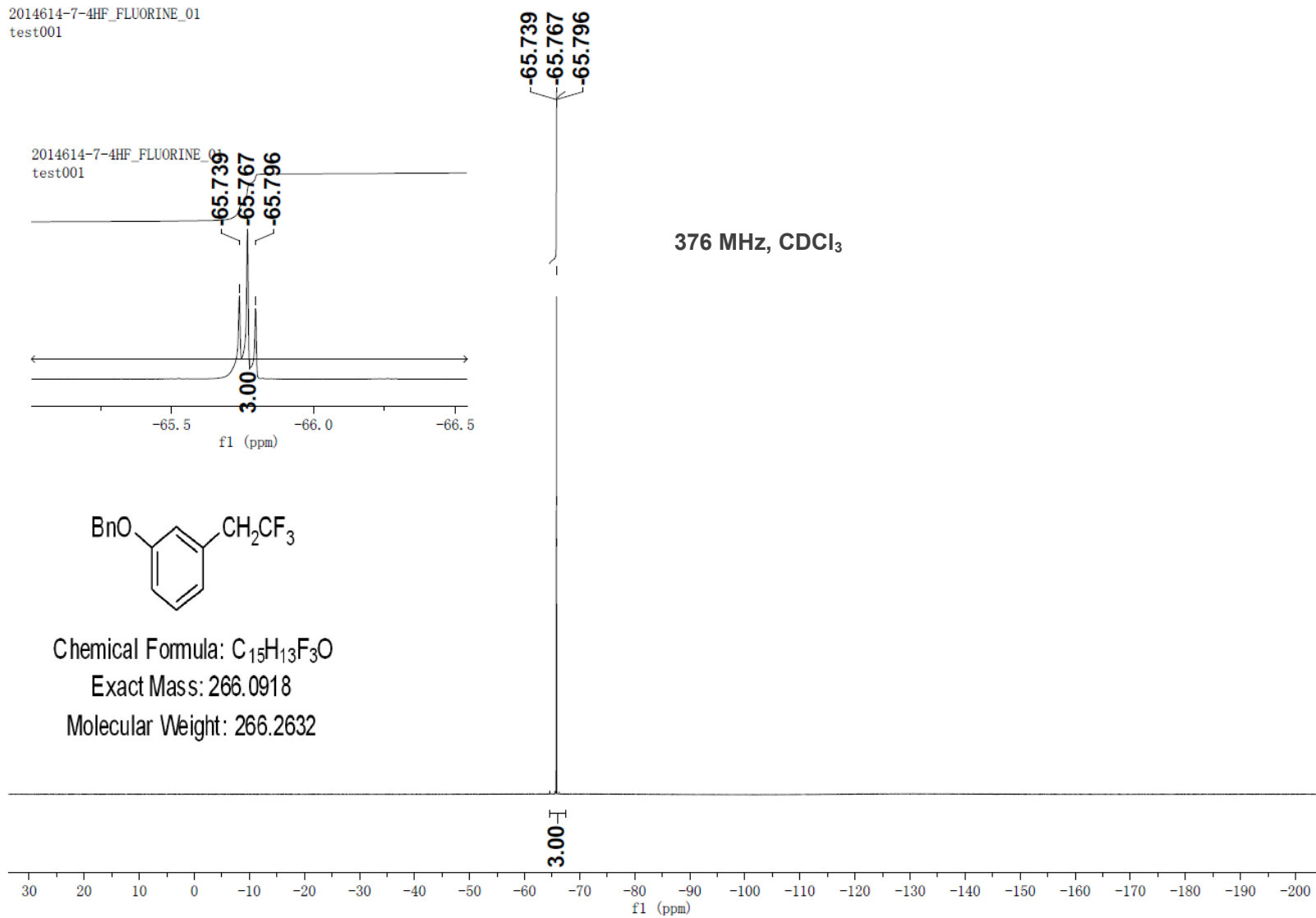

\section{4-(3-(2, 2, 2-Trifluoroethyl)phenyl)morpholine (12)}

2014614-7-3HF_PROTON_01 test001

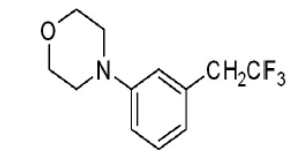

Chemical Formula: $\mathrm{C}_{12} \mathrm{H}_{14} \mathrm{~F}_{3} \mathrm{NO}$

Exact Mass: 245.1027

Molecular Weight: 245.2452

$400 \mathrm{MHz}^{\mathrm{CDCl}_{3}}$

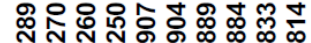

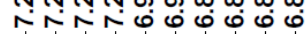
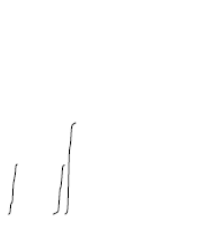

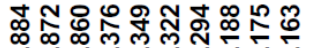

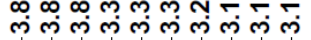

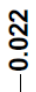

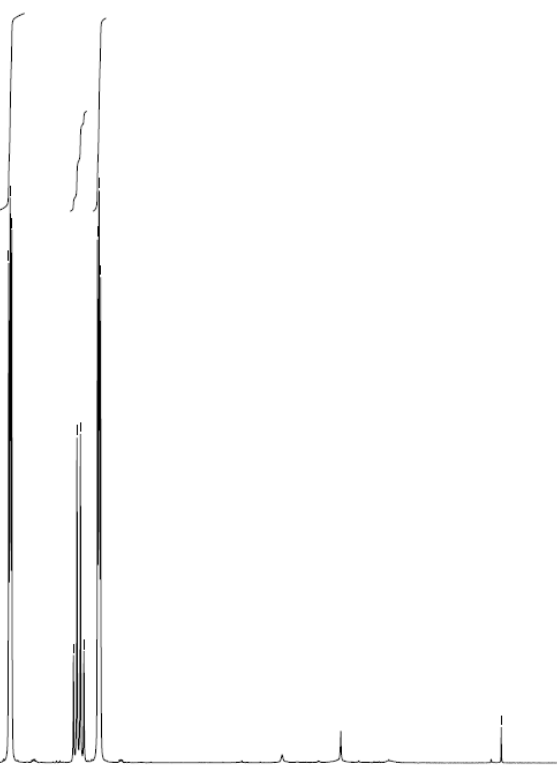

'ำ 엉워

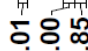

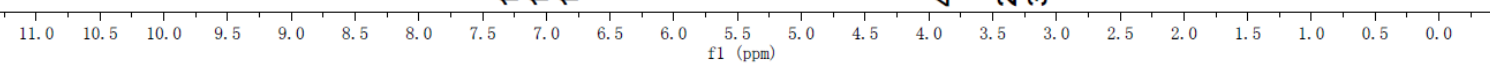


2014614-7-3-C_pslabel_01

test3

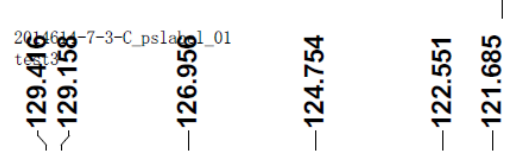

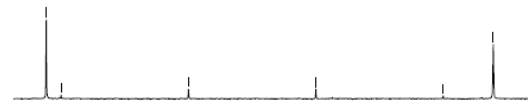

$126 \mathrm{MHz}^{\mathrm{CDCl}_{3}}$
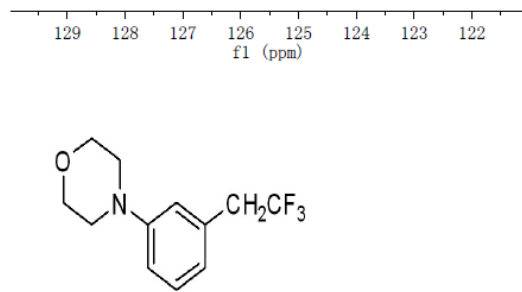

Chemical Formula: $\mathrm{C}_{12} \mathrm{H}_{14} \mathrm{~F}_{3} \mathrm{NO}$

Exact Mass: 245.1027

Molecular Weight: 245.2452

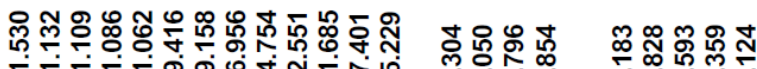

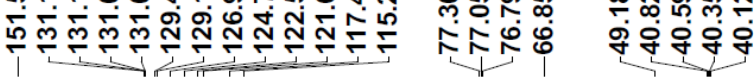
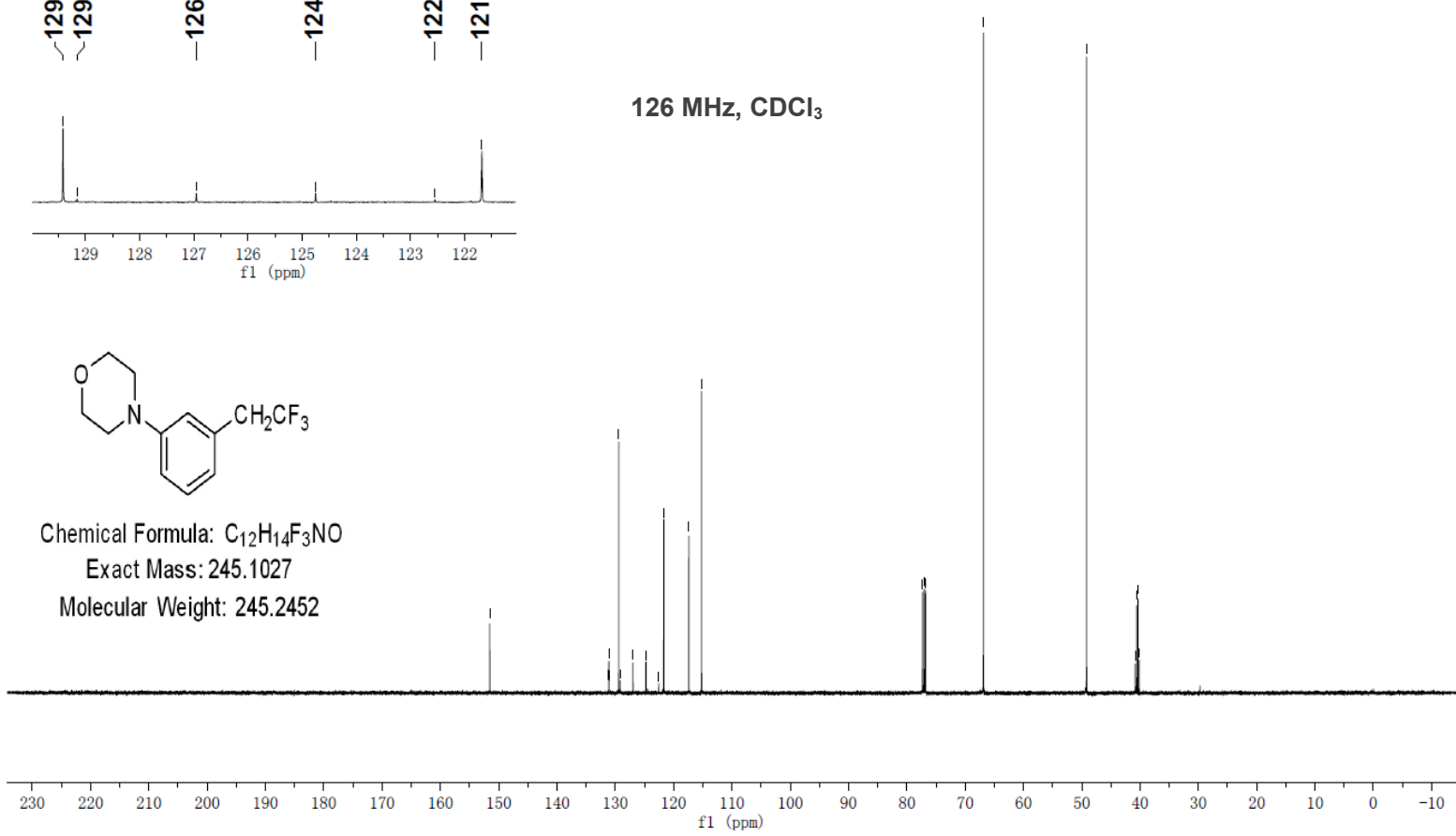

2014614-7-3HF_FLUORINE_01

test001

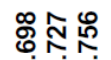

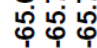

2014614-7-3HF_FLUORINE_0

test001

路员

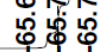

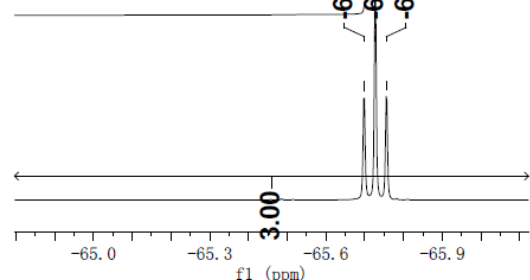

$376 \mathrm{MHz}, \mathrm{CDCl}_{3}$

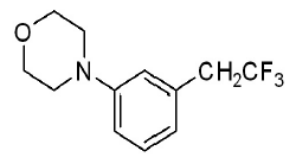

Chemical Formula: $\mathrm{C}_{12} \mathrm{H}_{14} \mathrm{~F}_{3} \mathrm{NO}$

Exact Mass: 245.1027

Molecular Weight: 245.2452 
4-(2, 2, 2-Trifluoroethyl)benzaldehyde (13)
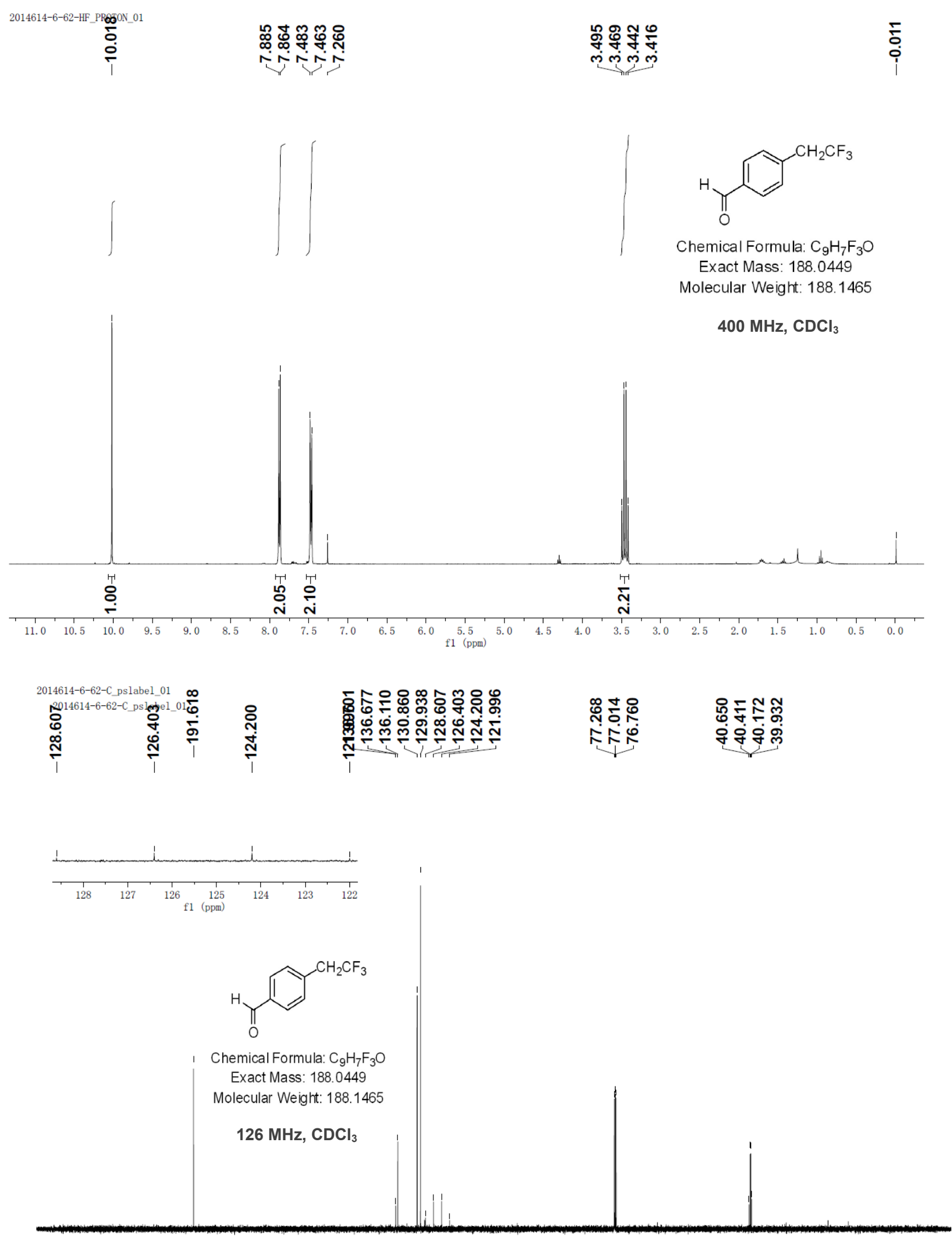

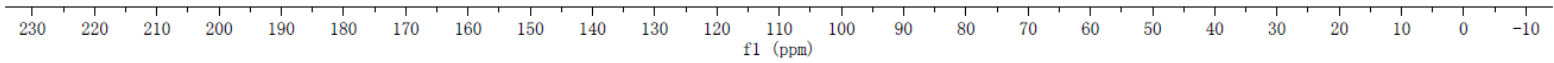




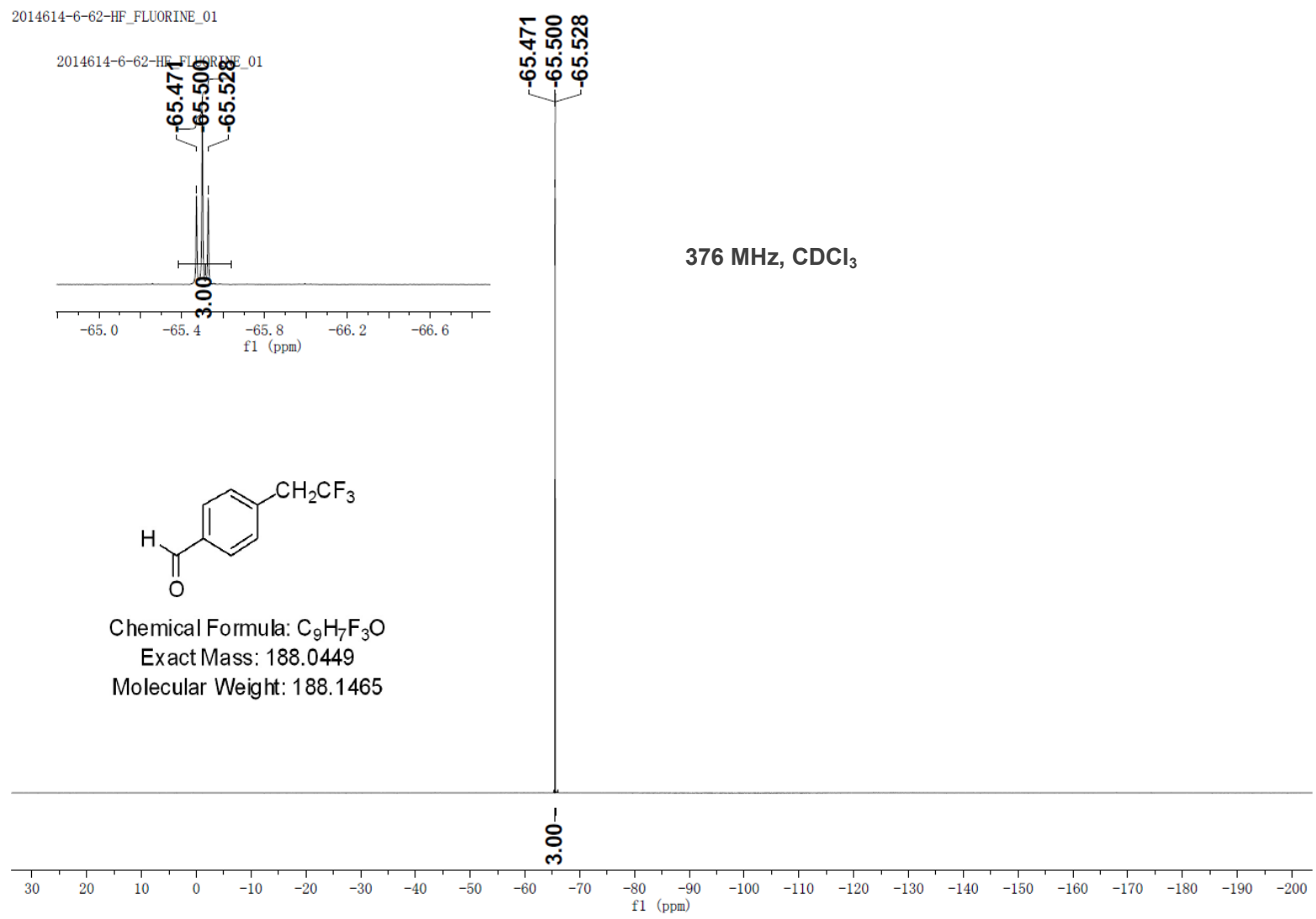

\section{3-(2, 2, 2-Trifluoroethyl)benzaldehyde (14)}

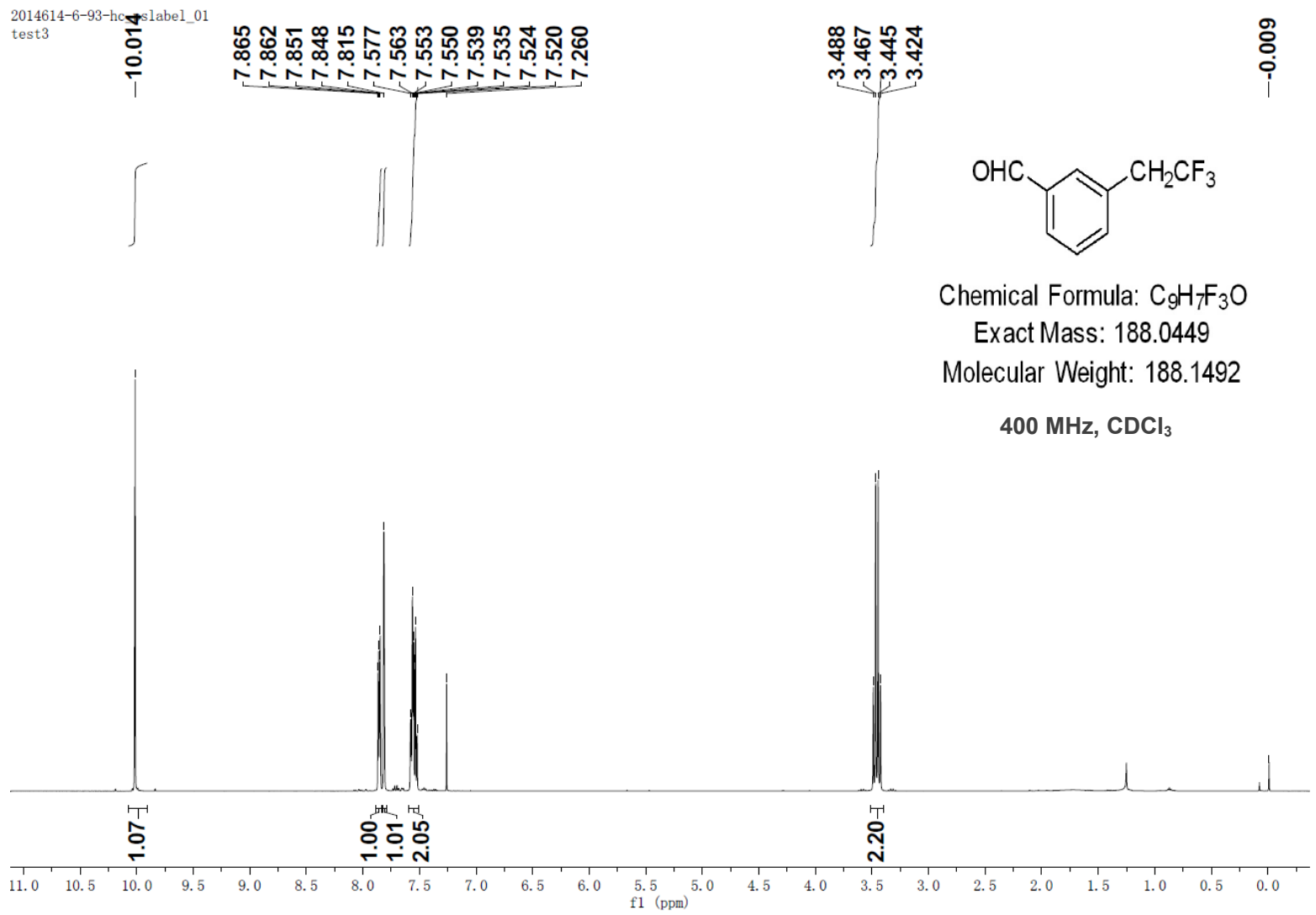



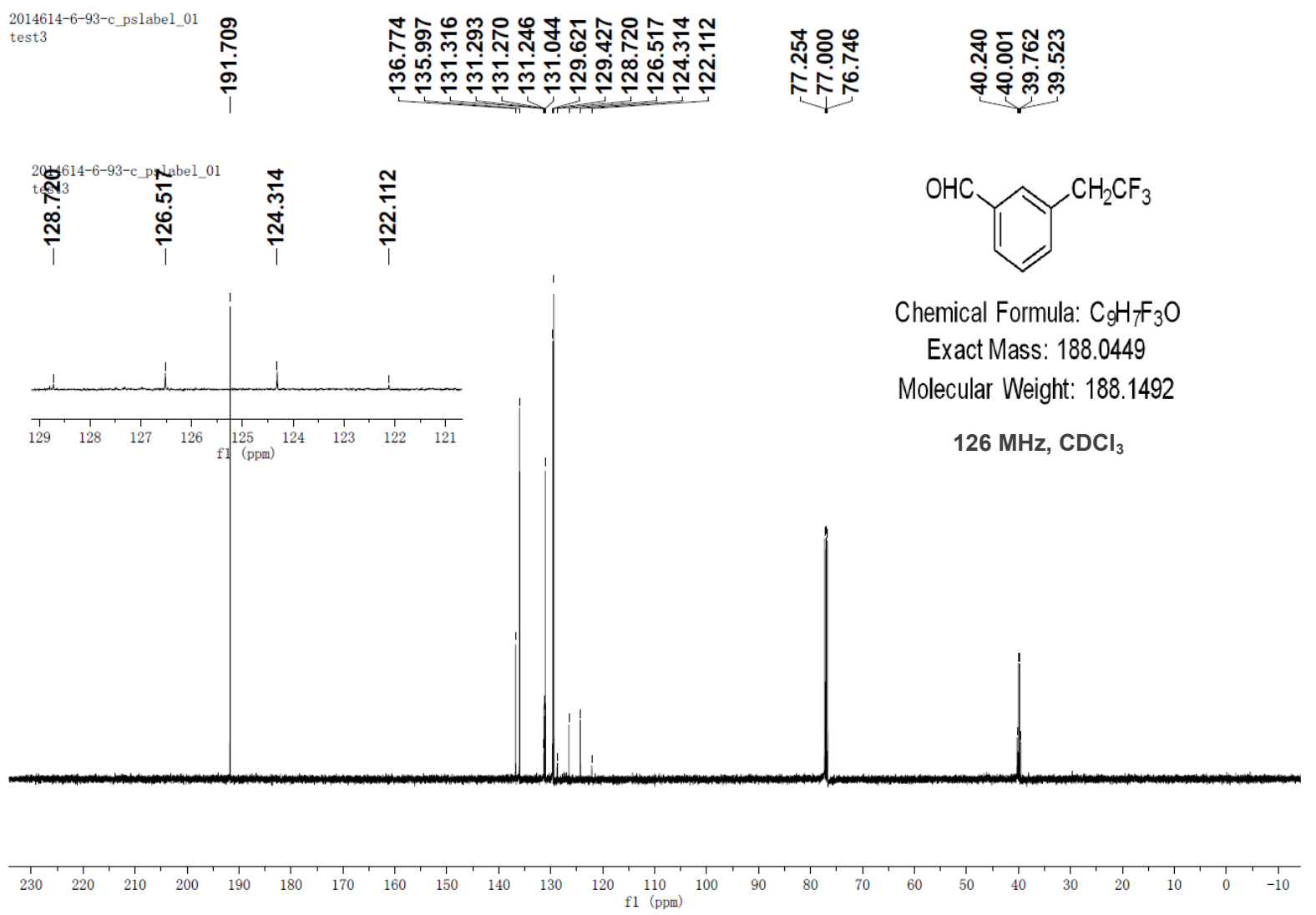

2014614-6-93F_FLUORINE_01

2014614-6-93F

\section{巡 땡}

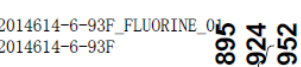

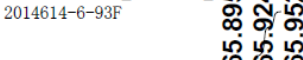

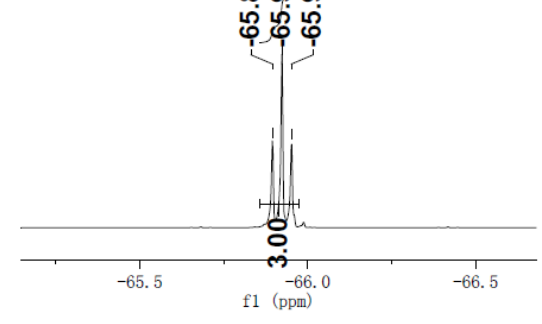

$376 \mathrm{MHz}^{\mathrm{CDCl}_{3}}$<smiles>O=Cc1cccc(CC[AsH3])c1</smiles>

Chemical Formula: $\mathrm{C}_{9} \mathrm{H}_{7} \mathrm{~F}_{3} \mathrm{O}$

Exact Mass: 188.0449

Molecular Weight: 188.1492

\section{$\dot{8}$}

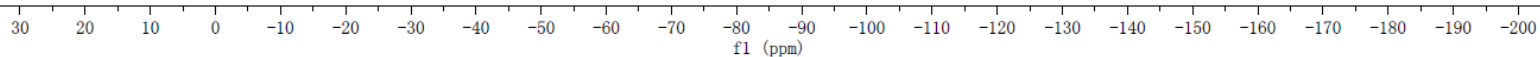



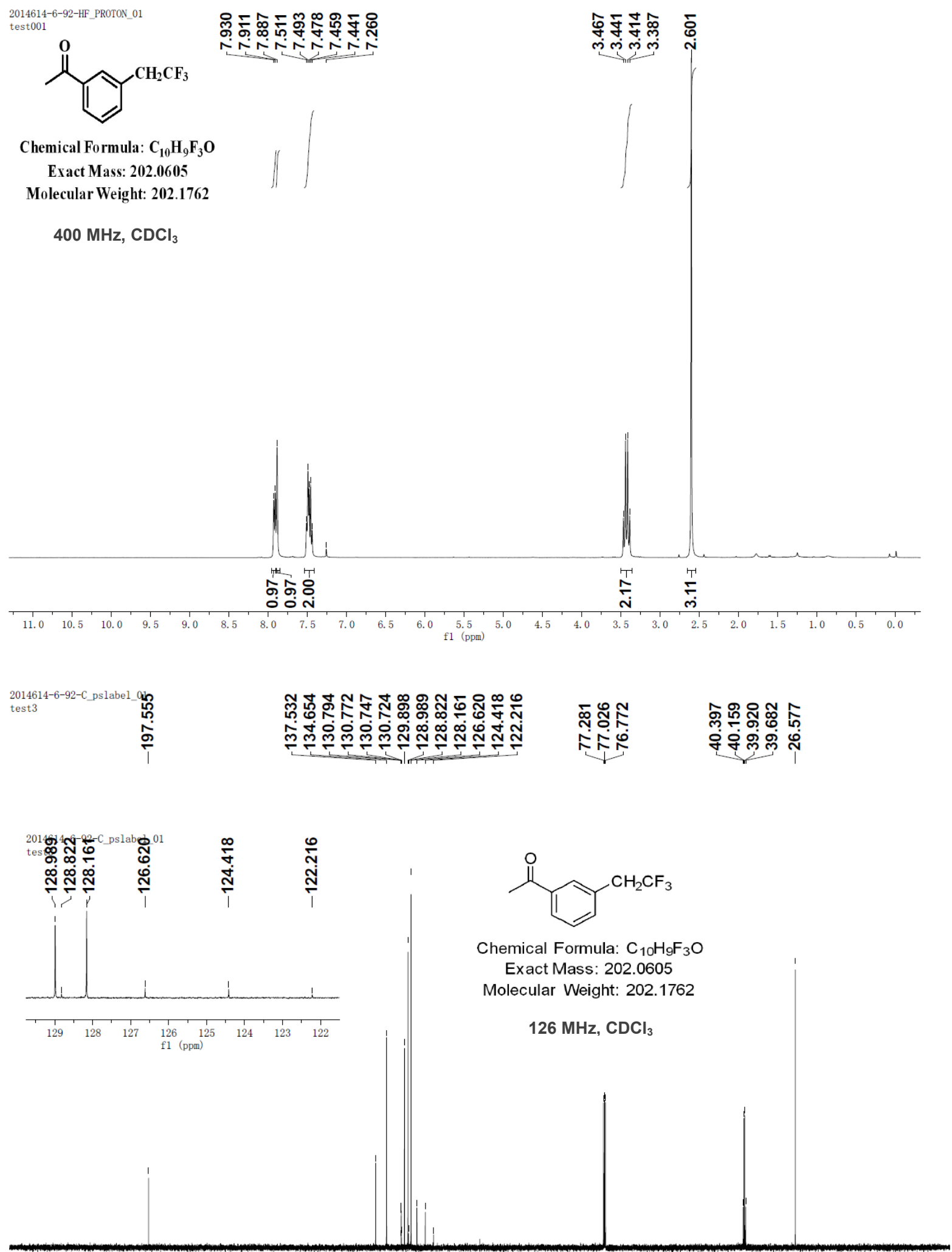

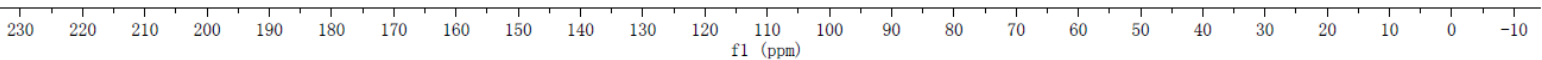


2014614-6-92-HF FLUORINE 01

test001

2014614-6-92-HF_FLUORINE NN

test001 ㅇํำ

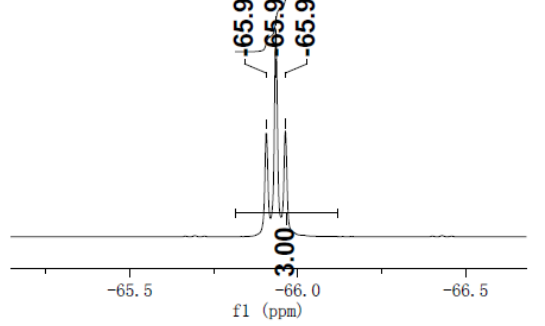

$\underbrace{\mathrm{CH}_{2} \mathrm{CF}_{3}}$

Chemical Formula: $\mathrm{C}_{10} \mathrm{H}_{9} \mathrm{~F}_{3} \mathrm{O}$

Exact Mass: 202.0605

Molecular Weight: 202.1762

\section{ㅎํ융}

$376 \mathrm{MHz}, \mathrm{CDCl}_{3}$

\section{8}

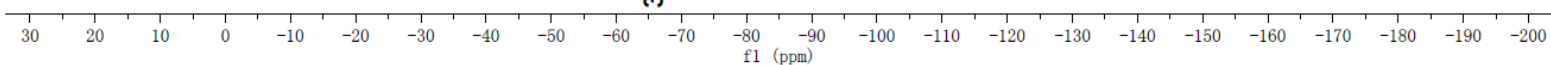

Ethyl 4-(2, 2, 2-trifluoroethyl)benzoate (16)

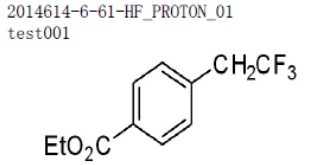

Chemical Formula: $\mathrm{C}_{11} \mathrm{H}_{11} \mathrm{~F}_{3} \mathrm{O}_{2}$

Exact Mass: 232.0711

Molecular Weight: 232.2022

$400 \mathrm{MHz}, \mathrm{CDCl}_{3}$

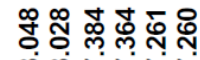

$\infty \infty N N$

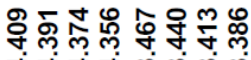

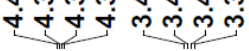
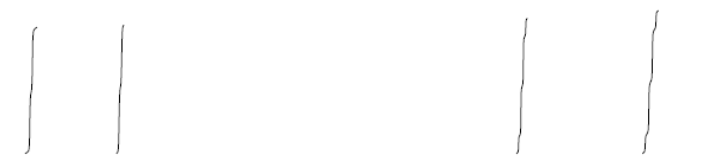

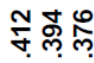

§ั
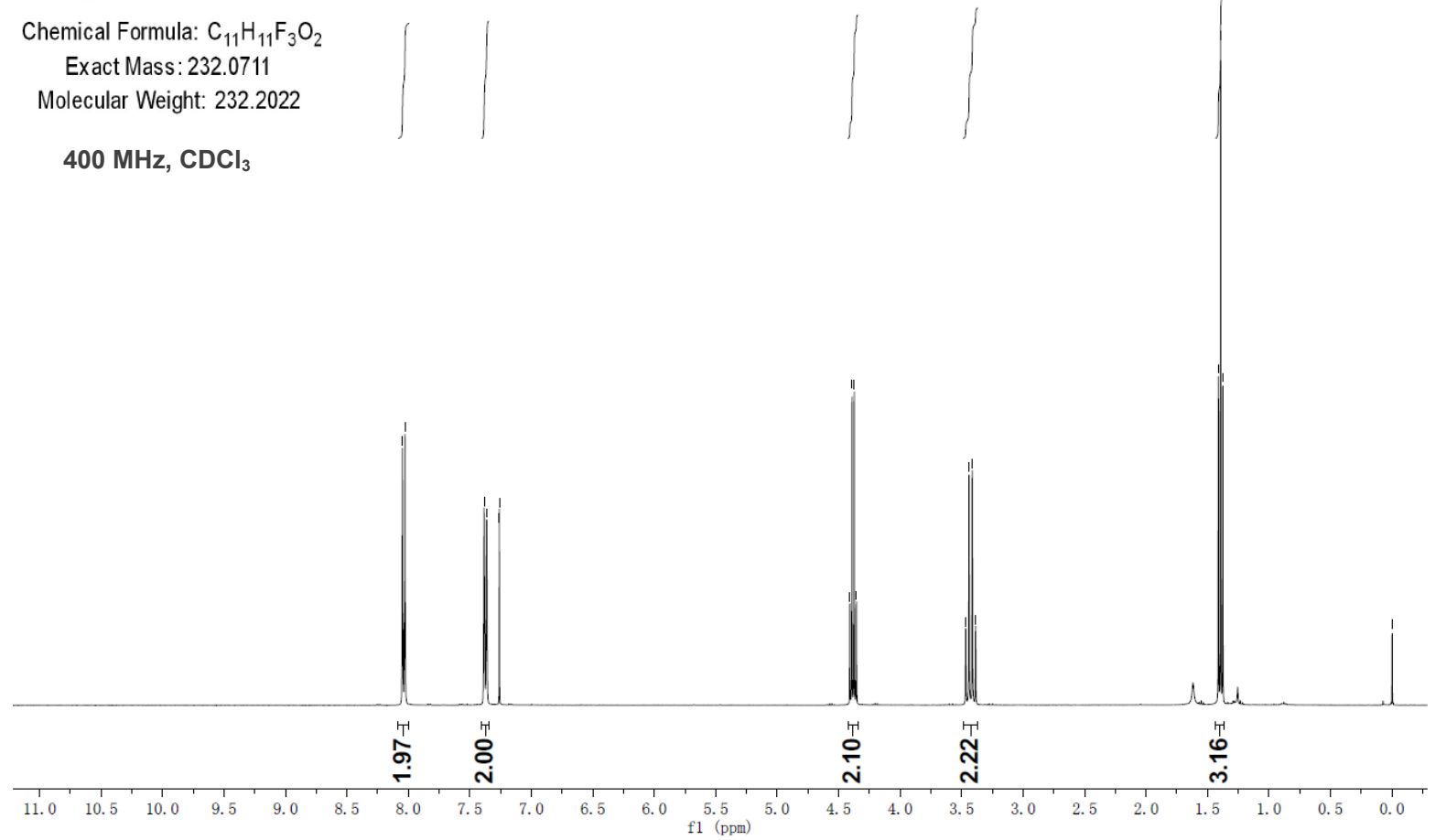

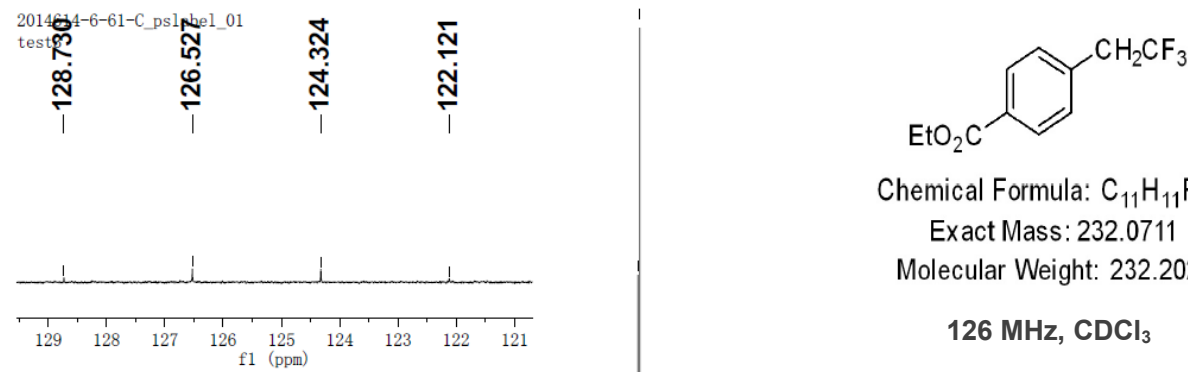

Chemical Formula: $\mathrm{C}_{11} \mathrm{H}_{11} \mathrm{~F}_{3} \mathrm{O}_{2}$

Exact Mass: 232.0711

Molecular Weight: 232.2022

$126 \mathrm{MHz}, \mathrm{CDCl}_{3}$
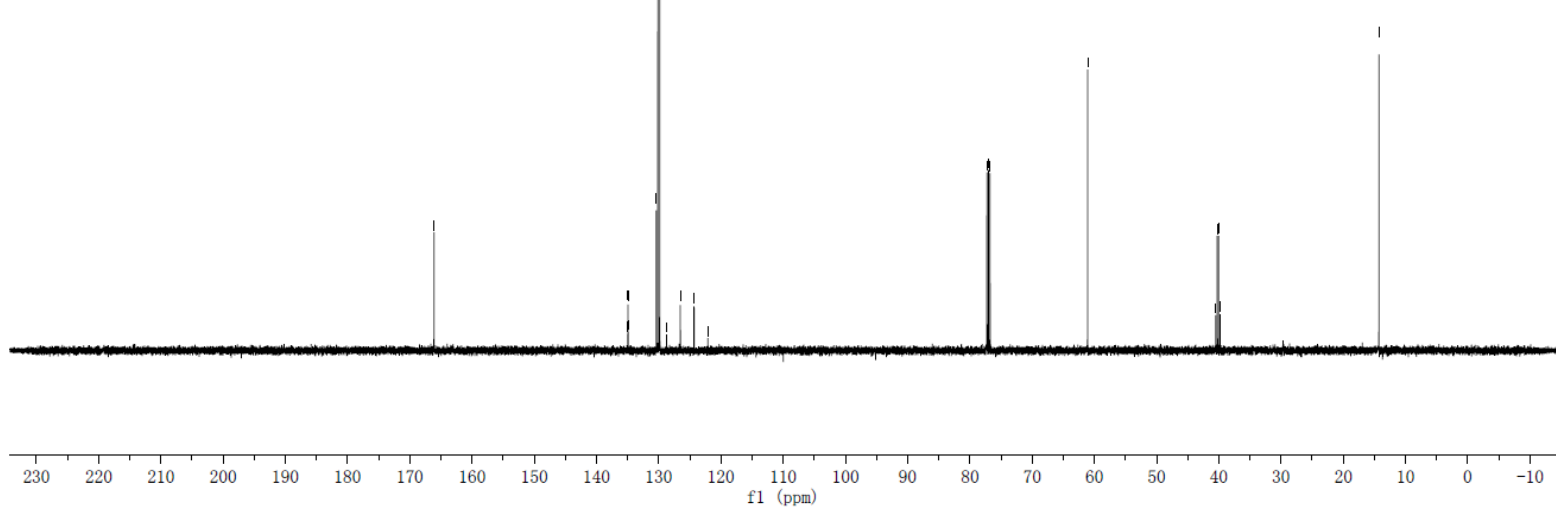

2014614-6-61-HF_FLUORINE_01 test001

2014614-6-61-HF_FLLeb10 an

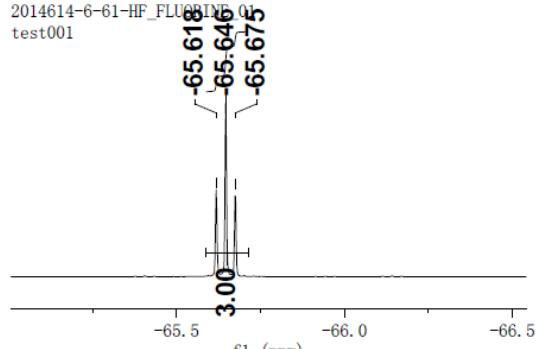

$376 \mathrm{MHz}, \mathrm{CDCl}_{3}$

f1 (ppm)

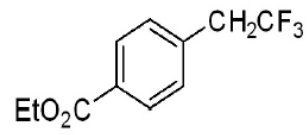

Chemical Formula: $\mathrm{C}_{11} \mathrm{H}_{11} \mathrm{~F}_{3} \mathrm{O}_{2}$

Exact Mass: 232.0711

Molecular Weight: 232.2022

\section{8}

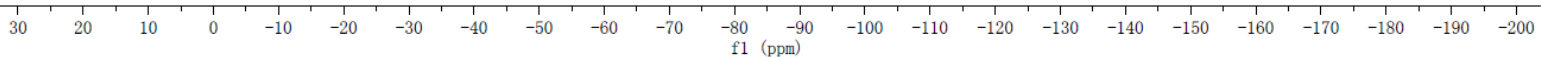


Ethyl 3-(2, 2, 2-trifluoroethyl)benzoate (17)

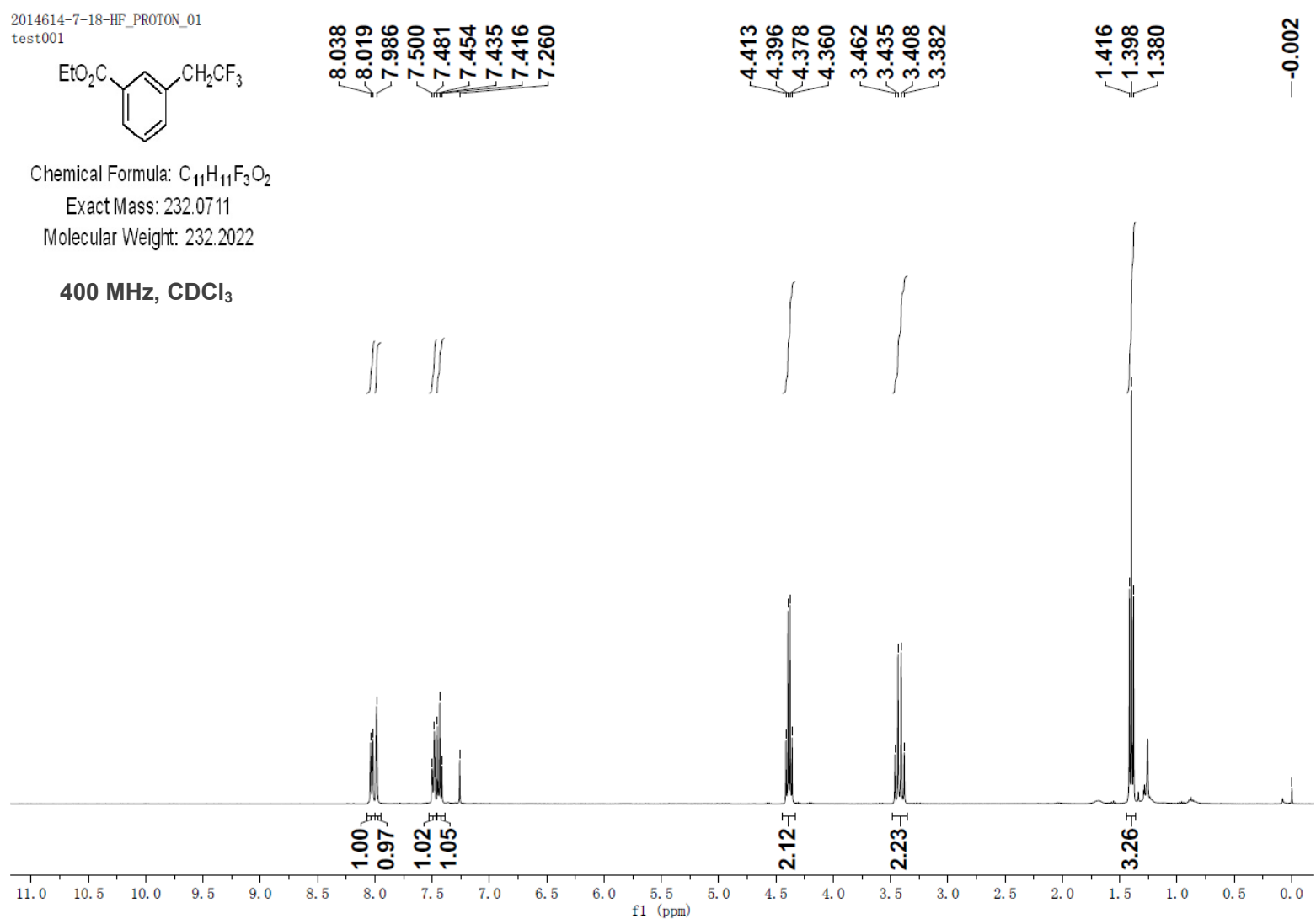

2014614-7-18-C_pslabel_01

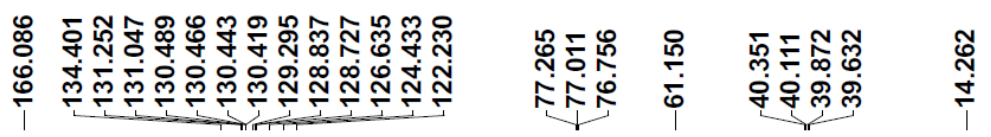

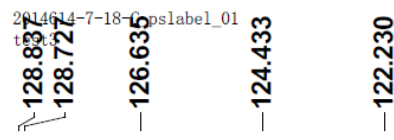

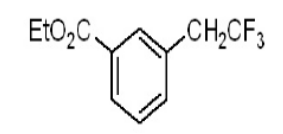

Chemical Formula: $\mathrm{C}_{11} \mathrm{H}_{11} \mathrm{~F}_{3} \mathrm{O}_{2}$

Exact Mass: 232.0711

Molecular Weight: 232.2022

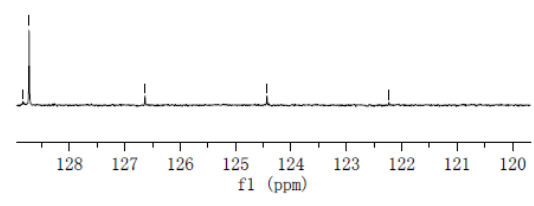

$126 \mathrm{MHz}, \mathrm{CDCl}_{3}$

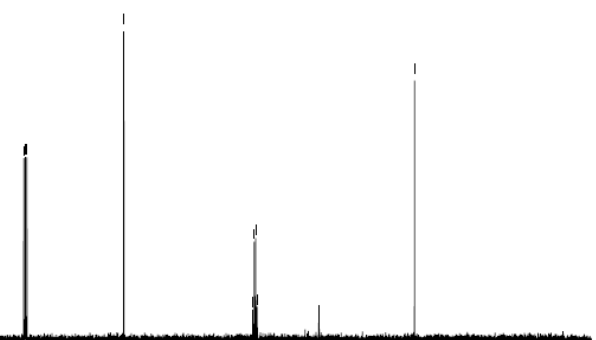

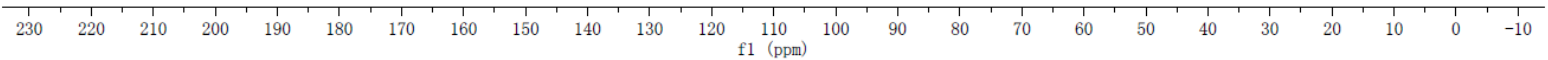


2014614-7-18-HF FLUORINE 01

test001

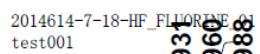

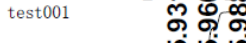

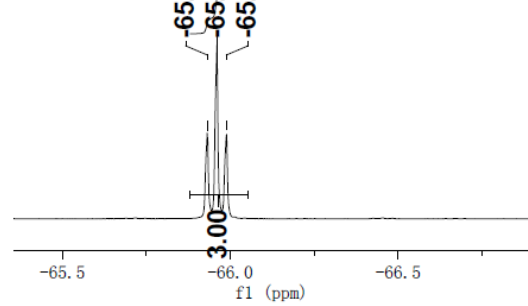

${ }^{\mathrm{EtO}_{2} \mathrm{C} Y \mathrm{CH}_{2} \mathrm{CF}_{3}}$

Chemical Formula: $\mathrm{C}_{11} \mathrm{H}_{11} \mathrm{~F}_{3} \mathrm{O}_{2}$

Exact Mass: 232.0711

Molecular Weight: 232.2022
$376 \mathrm{MHz}, \mathrm{CDCl}_{3}$

\section{8}

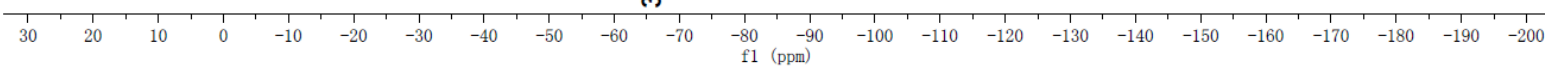

\section{2-(4-(2, 2, 2-Trifluoroethyl)phenyl)acetonitrile (18)}

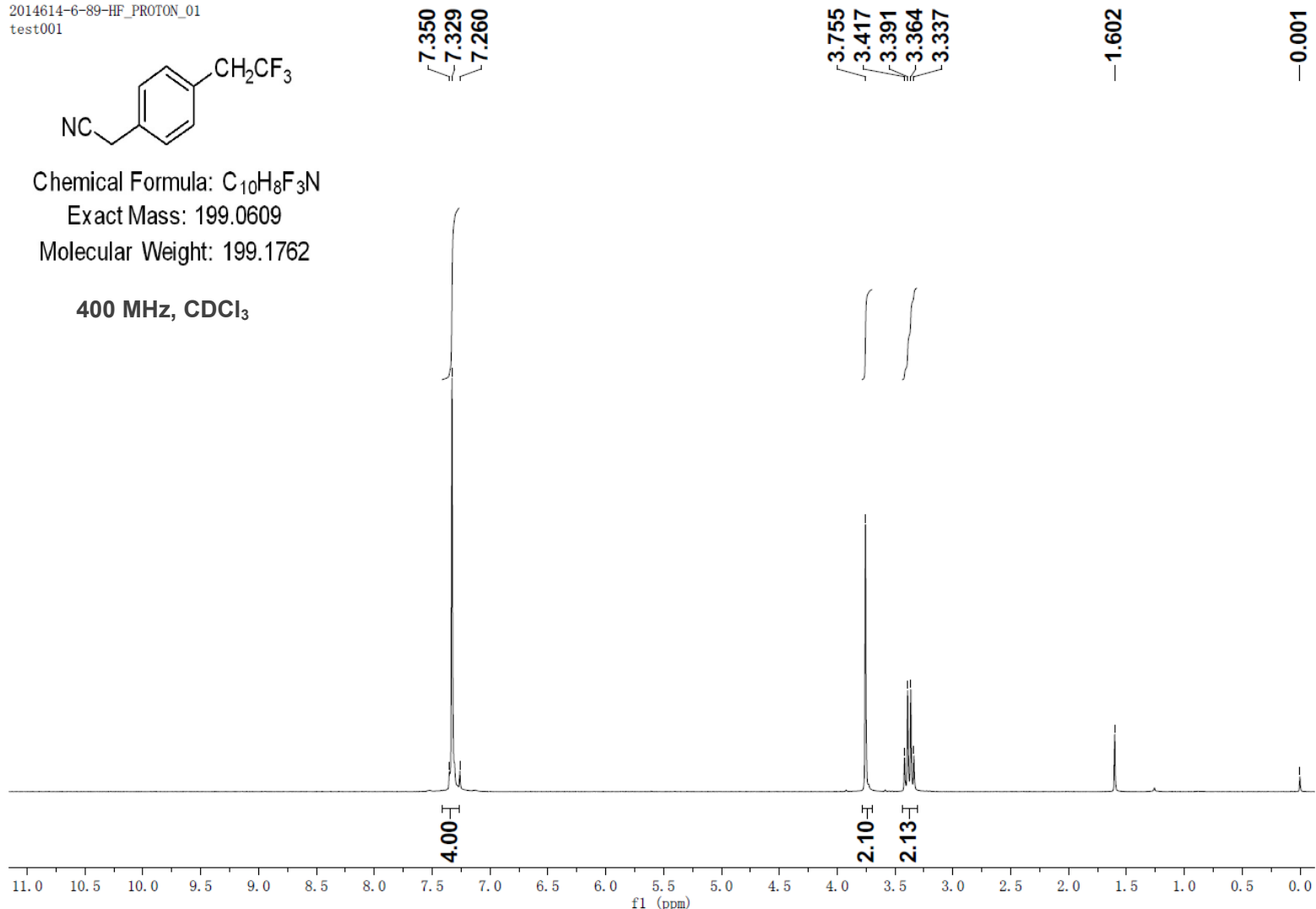




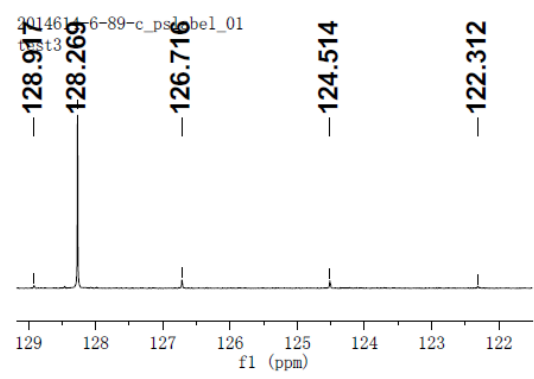

$126 \mathrm{MHz}, \mathrm{CDCl}_{3}$<smiles>N#CCc1ccc(CC(F)(F)F)cc1</smiles>

Chemical Formula: $\mathrm{C}_{10} \mathrm{H}_{8} \mathrm{~F}_{3} \mathrm{~N}$

Exact Mass: 199.0609

Molecular Weight: 199.1762

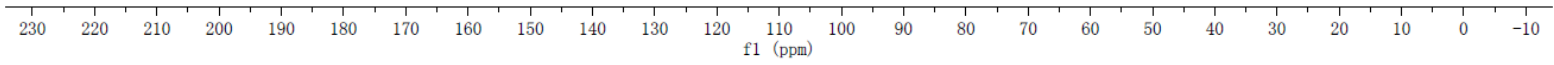

2014614-6-89-HF_FLUORINE_01 test001

\section{员哭}

फ़ि

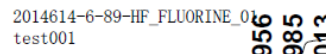

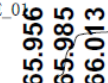

फ़ं

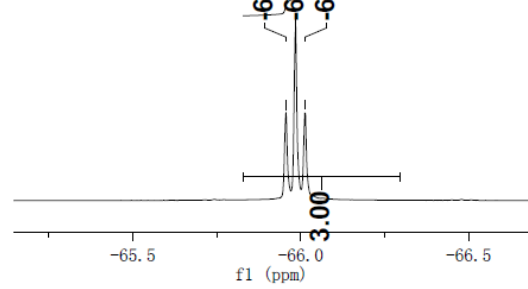

$376 \mathrm{MHz}, \mathrm{CDCl}_{3}$

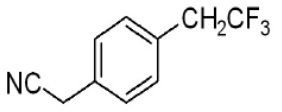

Chemical Formula: $\mathrm{C}_{10} \mathrm{H}_{8} \mathrm{~F}_{3} \mathrm{~N}$

Exact Mass: 199.0609

Molecular Weight: 199.1762 
(4-(2, 2, 2-Trifluoroethyl)phenyl)methanol (19)

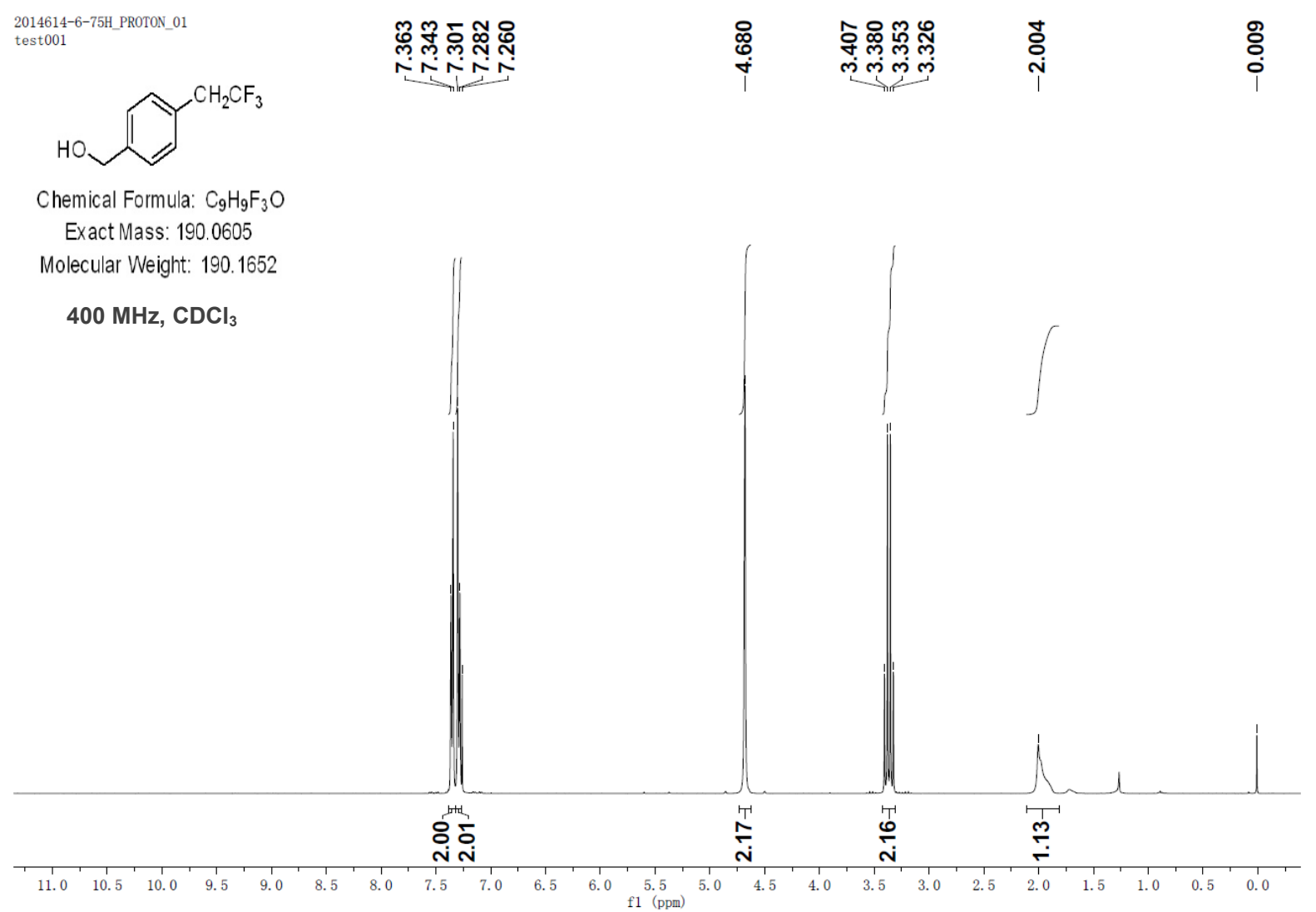

2014614-6-75-c_pslabel_01
test3
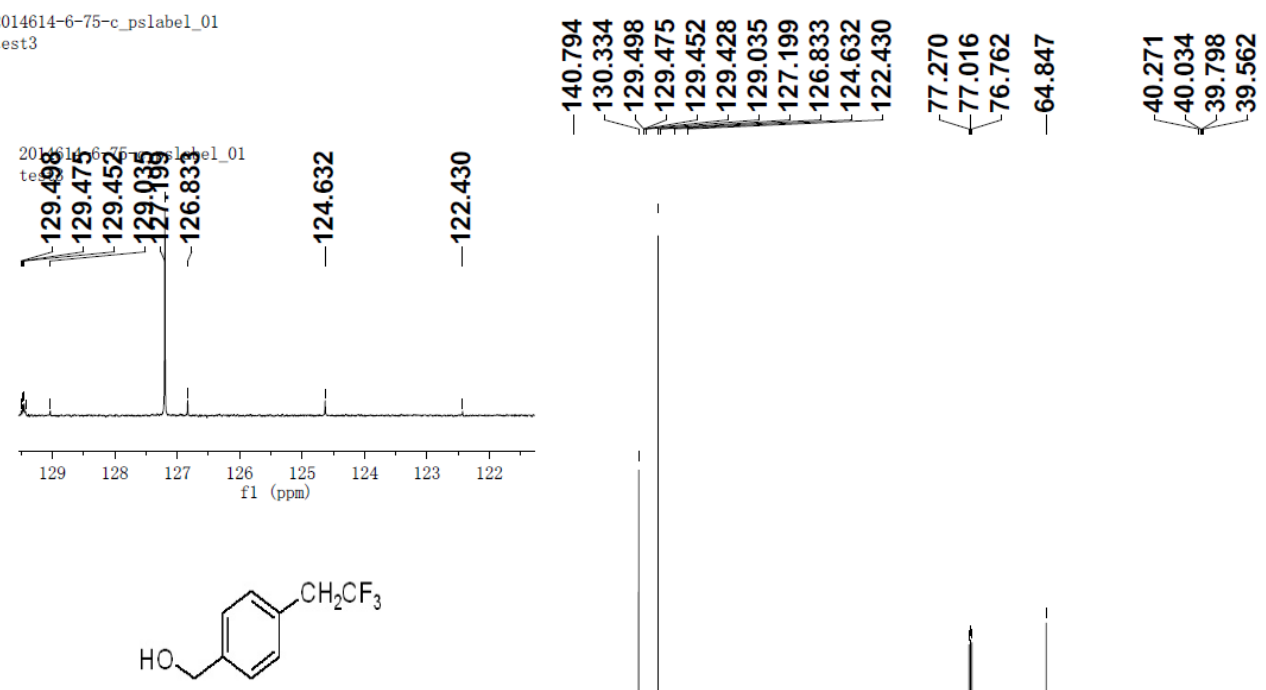

Chemical Formula: $\mathrm{C}_{9} \mathrm{H}_{9} \mathrm{~F}_{3} \mathrm{O}$

Exact Mass: 190.0605

Molecular Weight: 190.1652

$126 \mathrm{MHz}, \mathrm{CDCl}_{3}$

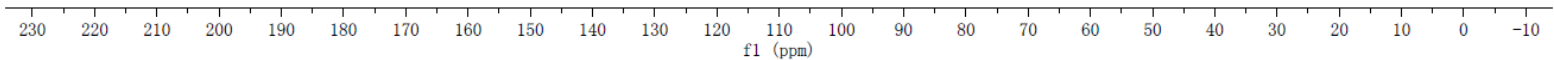


2014614-6-75-HF FLUORINE 01

test001

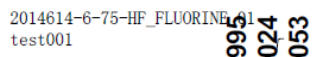

test001 융융

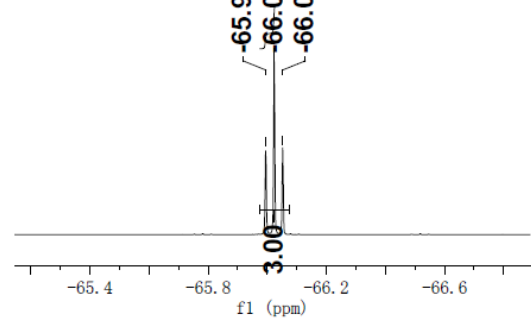

$\mathrm{CH}_{2} \mathrm{CF}_{3}$

Chemical Formula: $\mathrm{C}_{9} \mathrm{H}_{9} \mathrm{~F}_{3} \mathrm{O}$

Exact Mass: 190.0605

Molecular Weight: 190.1652

\section{边㖞}

$376 \mathrm{MHz}, \mathrm{CDCl}_{3}$

\section{8}

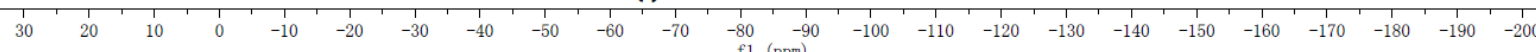

Trimethyl(4-(2, 2, 2-trifluoroethyl)phenyl)silane (20)
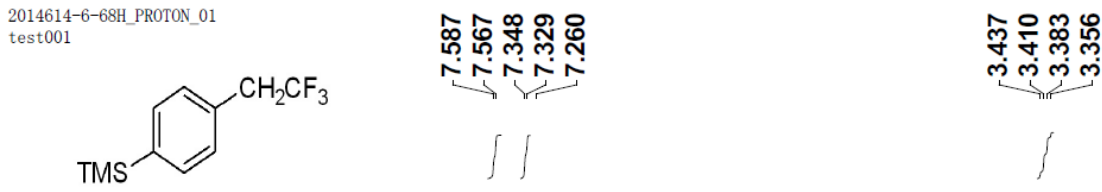

Chemical Formula: $\mathrm{C}_{11} \mathrm{H}_{15} \mathrm{~F}_{3} \mathrm{~S}$

Exact Mass: 232.0895

Molecular Weight: 232.3212

$400 \mathrm{MHz}, \mathrm{CDCl}_{3}$

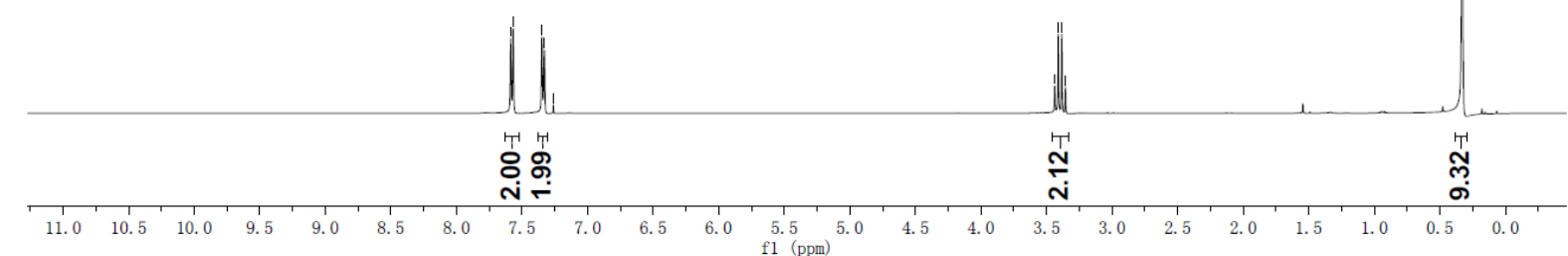




tes:

$126 \mathrm{MHz}, \mathrm{CDCl}_{3}$

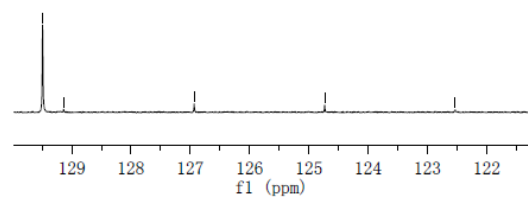<smiles>Cc1ccc(CC(F)(F)F)cc1</smiles>

Chemical Formula: $\mathrm{C}_{11} \mathrm{H}_{15} \mathrm{~F}_{3} \mathrm{Si}$

Exact Mass: 232.0895

Molecular Weight: 232.3212

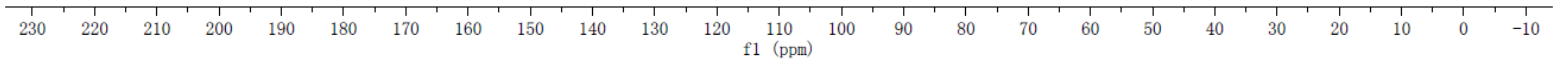

2014614-6-68-HF_FLUORINE_01 2014614-6-68-HF

2014614-6-68-HF FLGRTE CQ1

201461-6-68-HF

$376 \mathrm{MHz}, \mathrm{CDCl}_{3}$

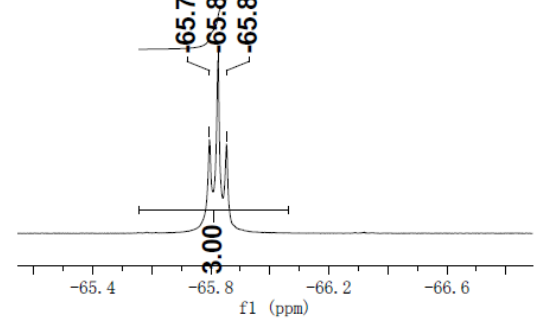

TMS<smiles>Cc1ccc(CC(F)(F)F)cc1</smiles>

Chemical Formula: $\mathrm{C}_{11} \mathrm{H}_{15} \mathrm{~F}_{3} \mathrm{Si}$

Exact Mass: 232.0895

Molecular Weight: 232.3212

\section{$\dot{8}$}

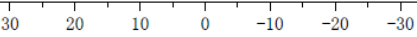

\begin{tabular}{|ccccccccccccccc|c|c|}
\hline & -70 & -80 & -90 & -100 & -110 & -120 & -130 & -140 & -150 & -160 & -170 & -180 & -190 & -200
\end{tabular} 
Trimethyl(3-(2, 2, 2-trifluoroethyl)phenyl)silane (21)
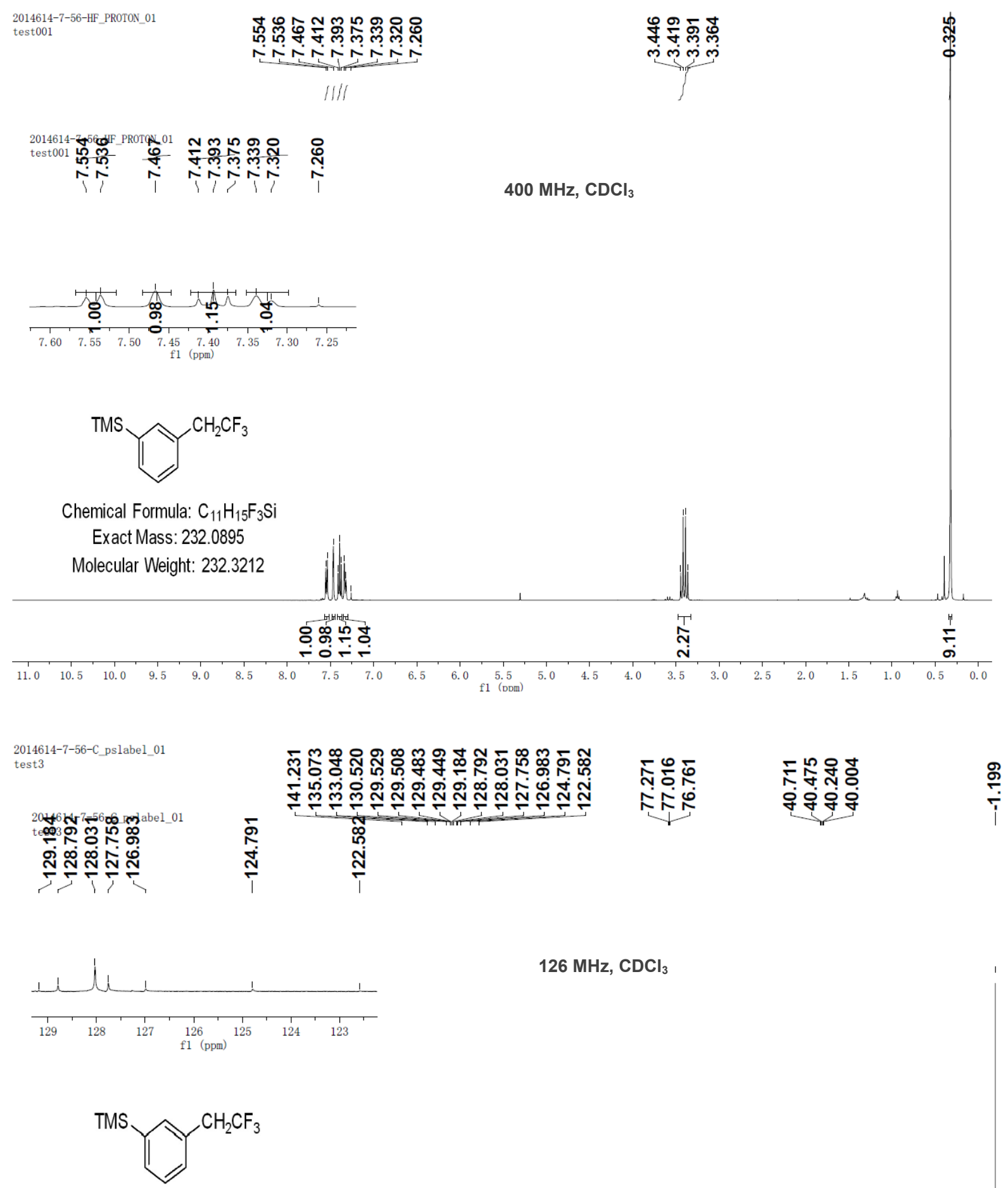

Chemical Formula: $\mathrm{C}_{11} \mathrm{H}_{15} \mathrm{~F}_{3} \mathrm{Si}$

Exact Mass: 232.0895

Molecular Weight: 232.3212

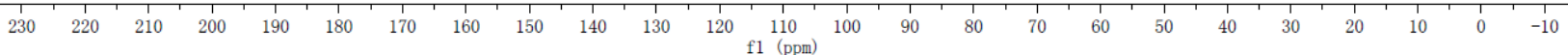




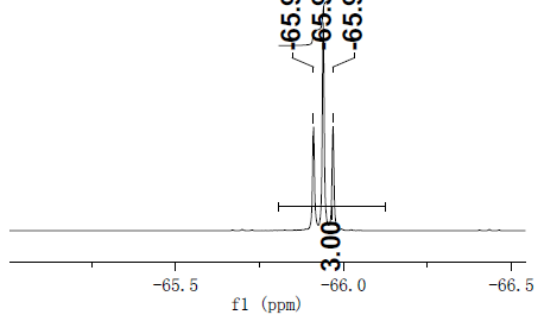

$376 \mathrm{MHz}, \mathrm{CDCl}_{3}$

TMS<smiles>Cc1cccc(CC(F)(F)F)c1</smiles>

Chemical Formula: $\mathrm{C}_{11} \mathrm{H}_{15} \mathrm{~F}_{3} \mathrm{Si}$

Exact Mass: 232.0895

Molecular Weight: 232.3212

\section{8}

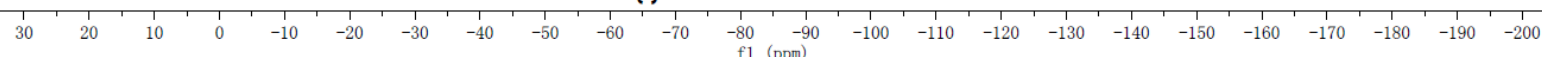

\section{4, 4, 5, 5-Tetramethyl-2-(4-(2, 2, 2-trifluoroethyl)phenyl)-1, 3, 2-dioxaborolane (22)}

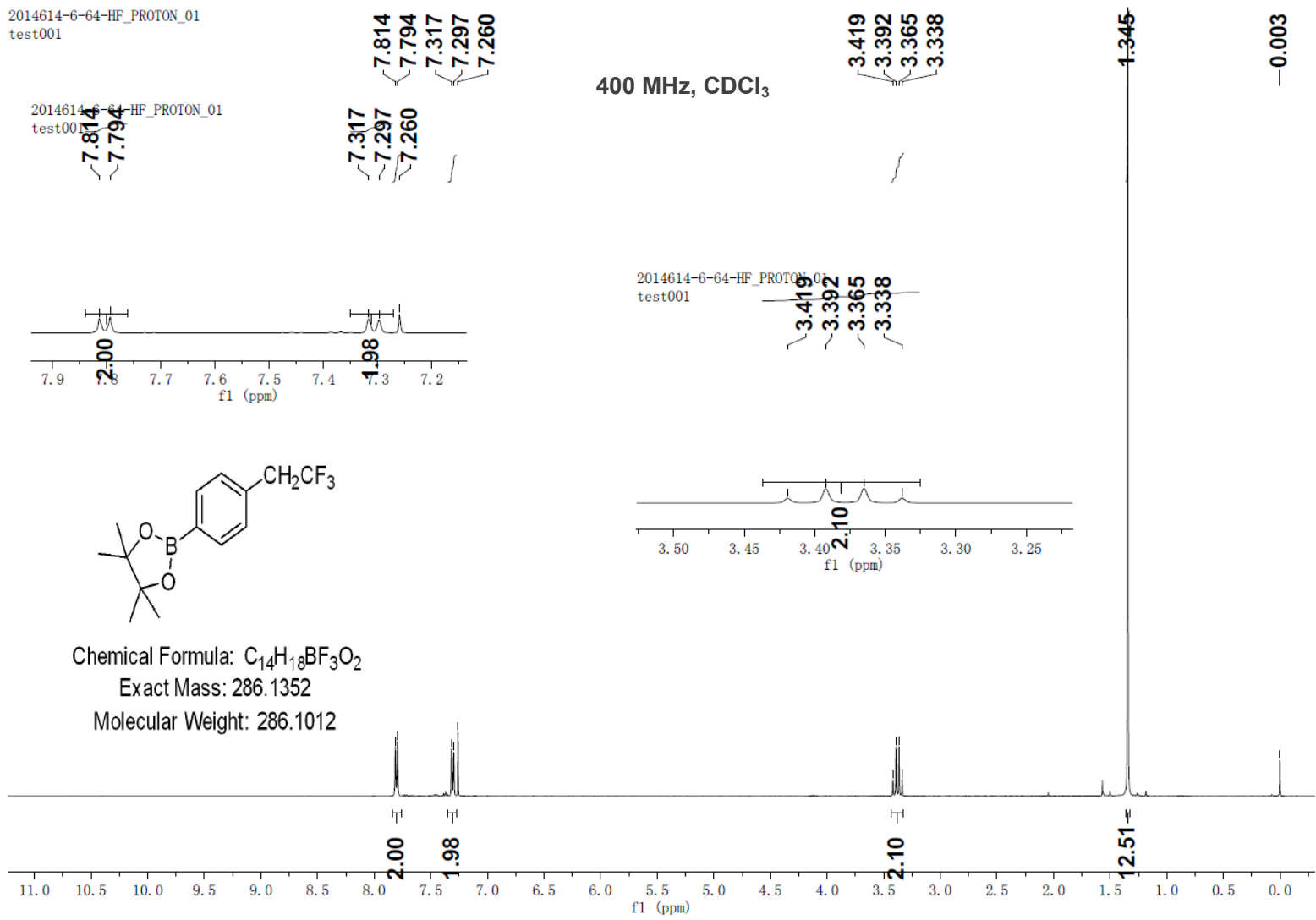


2014614-6-64-c_pslabel_01

test3

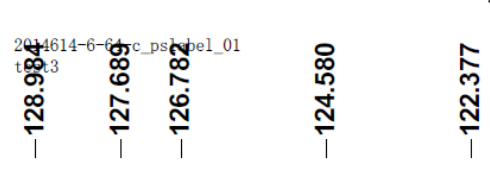

$126 \mathrm{MHz}, \mathrm{CDCl}_{3}$

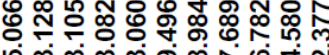

N

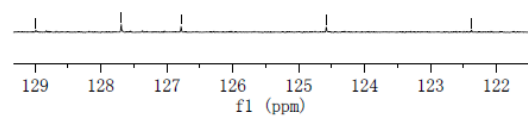

施总旅

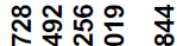

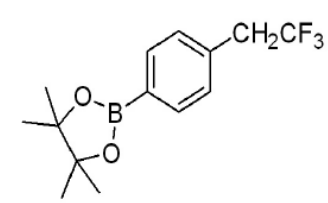

Chemical Formula: $\mathrm{C}_{14} \mathrm{H}_{18} \mathrm{BF}_{3} \mathrm{O}_{2}$

Exact Mass: 286.1352

Molecular Weight: 286.1012

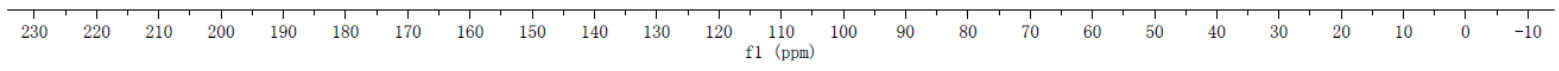

2014614-6-64-HF_FLUORINE_01

test001

\section{ป}

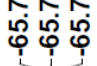

2014614-6-64-HF_FLUORINE_0 test001

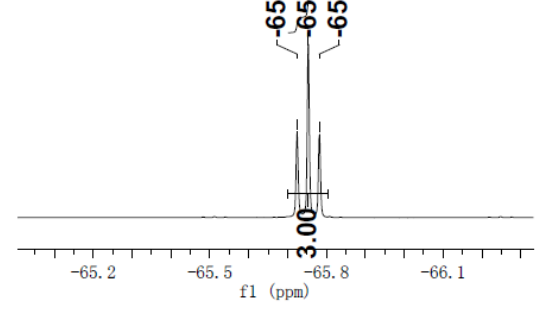

$376 \mathrm{MHz}, \mathrm{CDCl}_{3}$

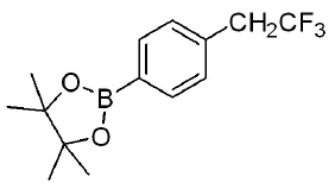

Chemical Formula: $\mathrm{C}_{14} \mathrm{H}_{18} \mathrm{BF}_{3} \mathrm{O}_{2}$

Exact Mass: 286.1352

Molecular Weight: 286.1012

\section{$\dot{8}$}

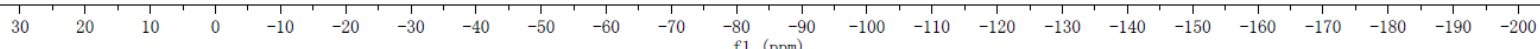


7-(2, 2, 2-Trifluoroethyl)naphthalen-2-ol (23)

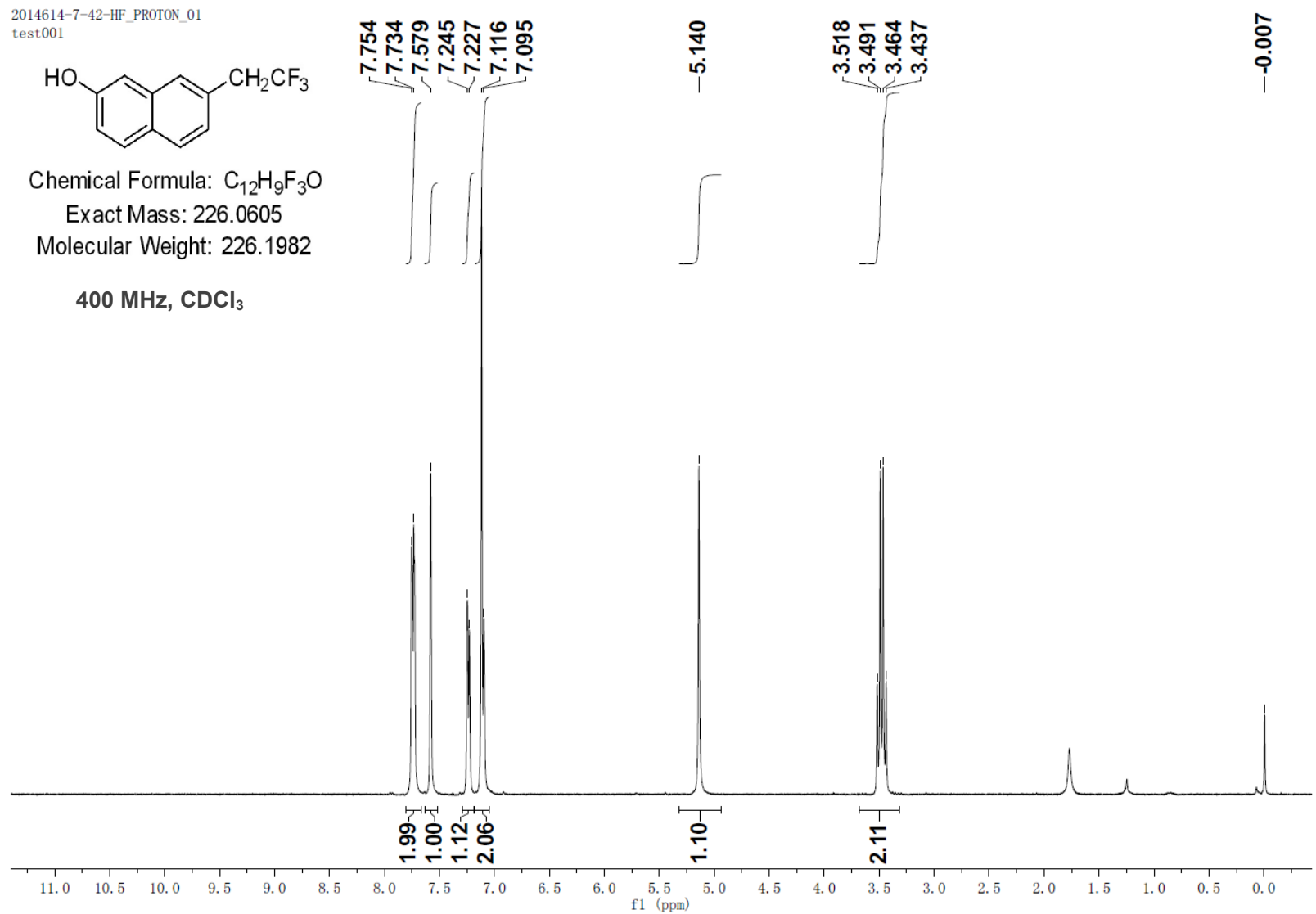

2014614-7-42-C_pslabe1_01 test3

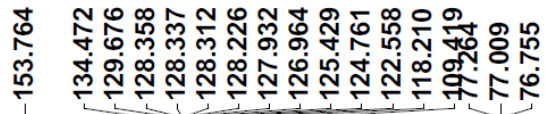

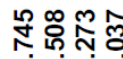

ค่ \& \&

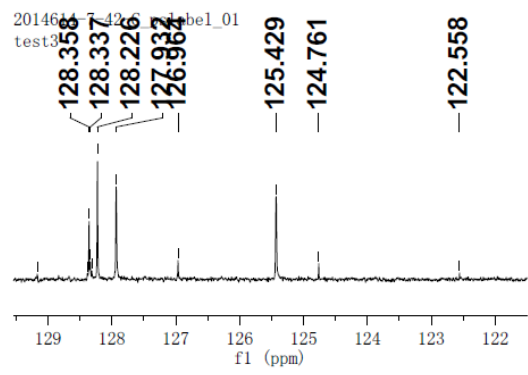

$\mathrm{CH}_{2} \mathrm{CF}_{3}$

Chemical Formula: $\mathrm{C}_{12} \mathrm{H}_{9} \mathrm{~F}_{3} \mathrm{O}$

Exact Mass: 226.0605

Molecular Weight: 226.1982

$126 \mathrm{MHz}, \mathrm{CDCl}_{3}$

$\begin{array}{lllllllllllll}230 & 220 & 210 & 200 & 190 & 180 & 170 & 160 & 150 & 140 & 130 & 120 & 110 \\ \mathrm{f1}(\mathrm{ppm}) & 100\end{array}$ 


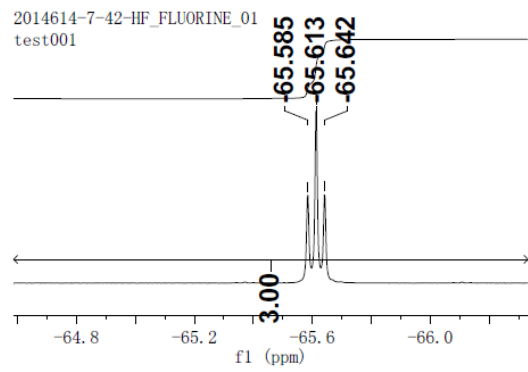

$376 \mathrm{MHz}, \mathrm{CDCl}_{3}$<smiles>Oc1ccc2ccc(CC(F)(F)F)cc2c1</smiles>

Chemical Formula: $\mathrm{C}_{12} \mathrm{H}_{9} \mathrm{~F}_{3} \mathrm{O}$

Exact Mass: 226.0605

Molecular Weight: 226.1982

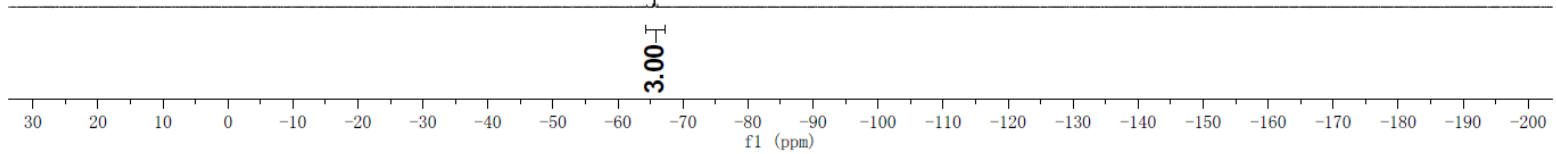

\section{2-(2, 2, 2-Trifluoroethyl)naphthalene (24)}

\section{4-6-60-HF_PROTON}

$\stackrel{\substack{0 \\ 0}}{i}$<smiles>FC(F)(F)Cc1ccc2ccccc2c1</smiles>

Chemical Formula: $\mathrm{C}_{12} \mathrm{H}_{9} \mathrm{~F}_{3}$

Exact Mass: 210.0656

Molecular Weight: 210.1992

$400 \mathrm{MHz}, \mathrm{CDCl}_{3}$

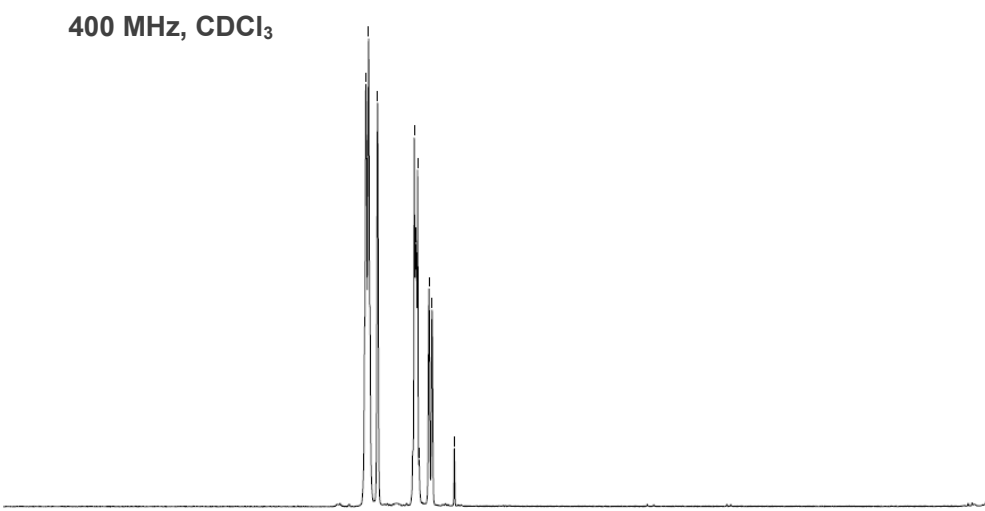

8.

$\stackrel{\infty}{-}$

$\begin{array}{lllllllllll}10.0 & 9.5 & 9.0 & 8.5 & 8.0 & 7.5 & 7.0 & 6.5 & 6.0 & 5.5 & \begin{array}{l}5.0 \\ \mathrm{f1}\end{array}(\mathrm{ppm})\end{array}$ 


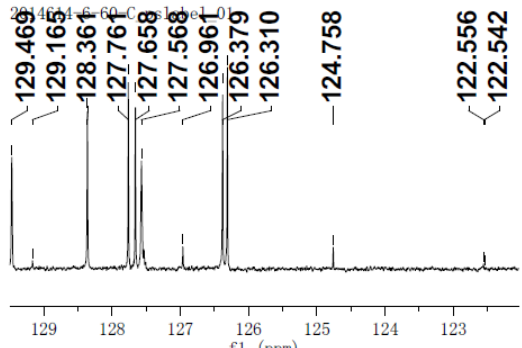

융ำ

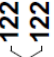

$126 \mathrm{MHz}, \mathrm{CDCl}_{3}$

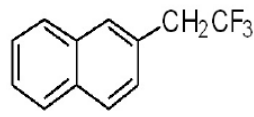

Chemical Formula: $\mathrm{C}_{12} \mathrm{H}_{9} \mathrm{~F}_{3}$

Exact Mass: 210.0656

Molecular Weight: 210.1992

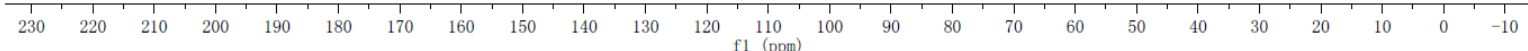

2014614-6-60-HF_FLUORINE 01

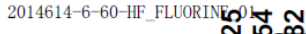

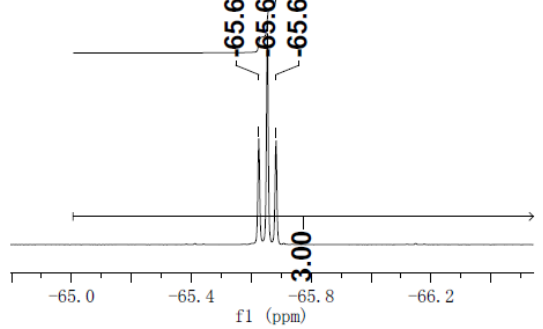

$376 \mathrm{MHz}, \mathrm{CDCl}_{3}$

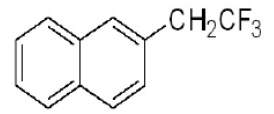

Chemical Formula: $\mathrm{C}_{12} \mathrm{H}_{9} \mathrm{~F}_{3}$

Exact Mass: 210.0656

Molecular Weight: 210.1992

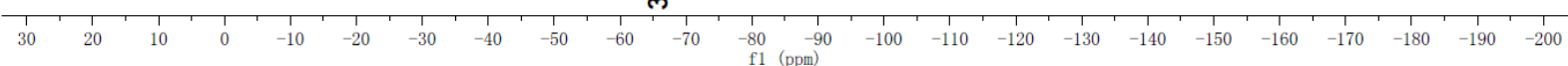


2-(2, 2, 2-Trifluoroethyl)-9H-fluorene (25)
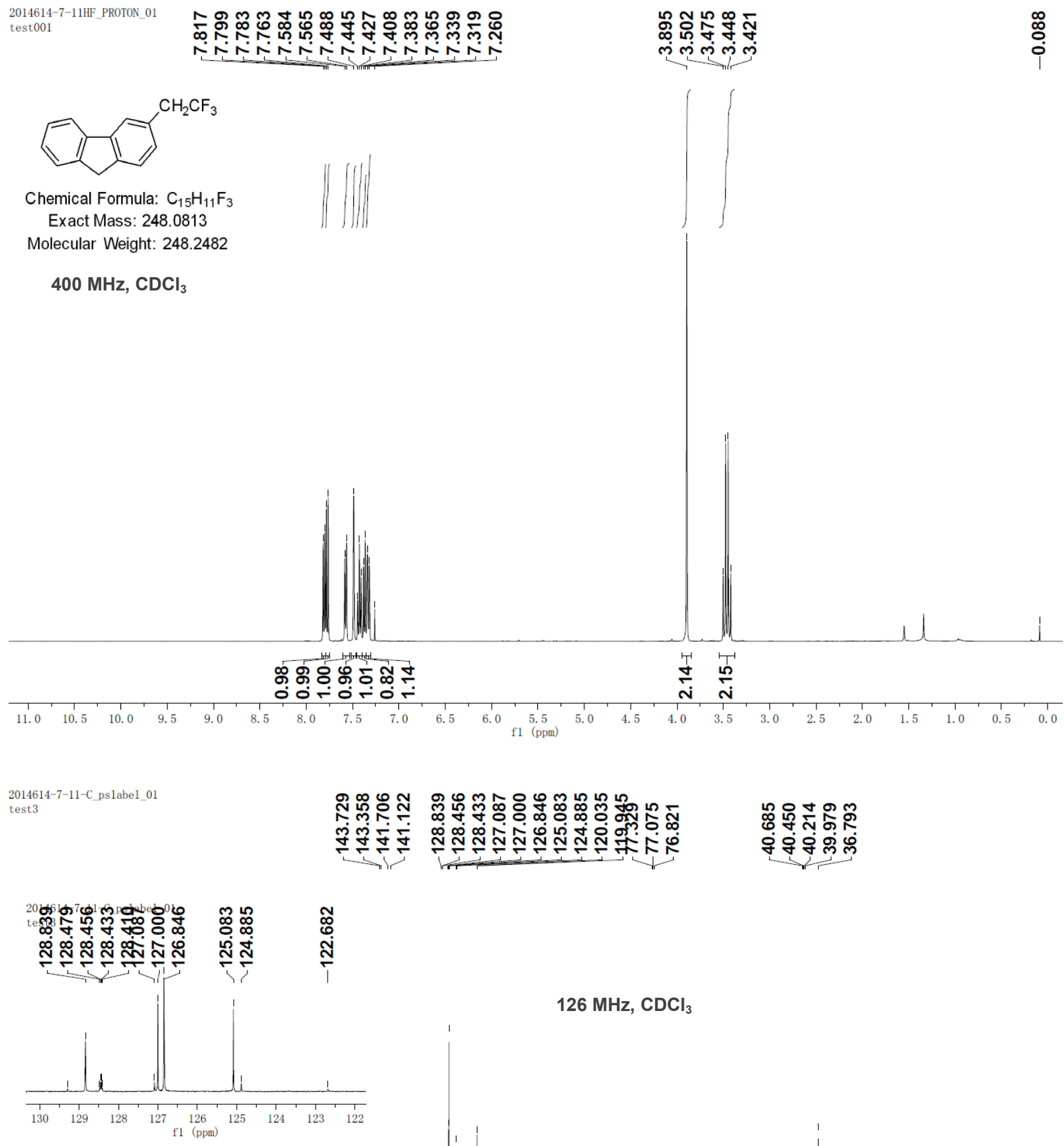

$126 \mathrm{MHz}, \mathrm{CDCl}_{3}$

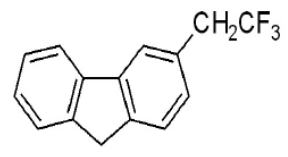

Chemical Formula: $\mathrm{C}_{15} \mathrm{H}_{11} \mathrm{~F}_{3}$

Exact Mass: 248.0813

Molecular Weight: 248.2482

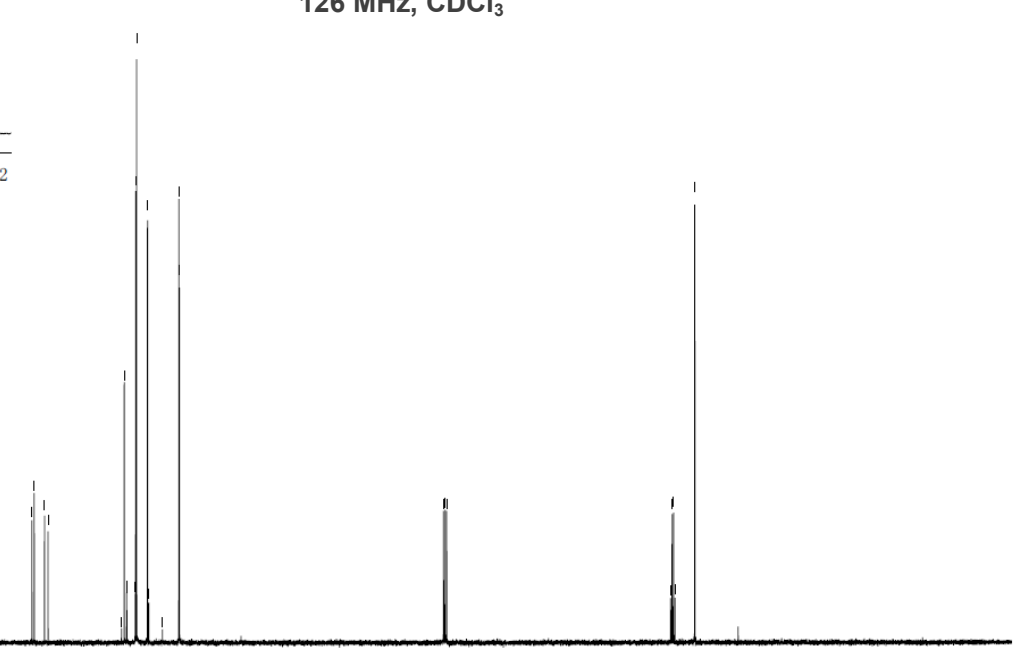

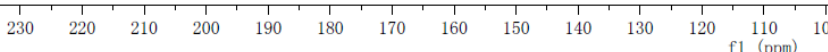


2014614-7-11HF_FLUORINE_01

test001

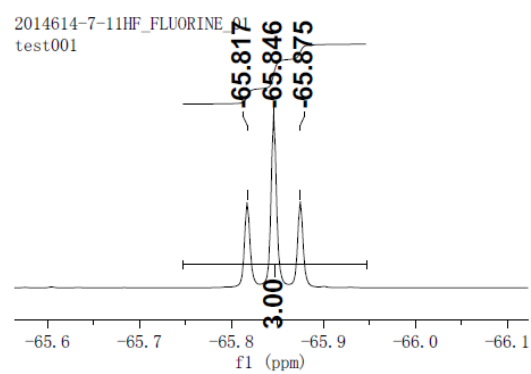

소에

पृ

$376 \mathrm{MHz}, \mathrm{CDCl}_{3}$

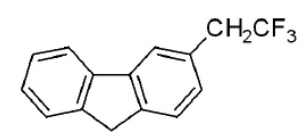

Chemical Formula: $\mathrm{C}_{15} \mathrm{H}_{11} \mathrm{~F}_{3}$

Exact Mass: 248.0813

Molecular Weight: 248.2482

\section{\&}

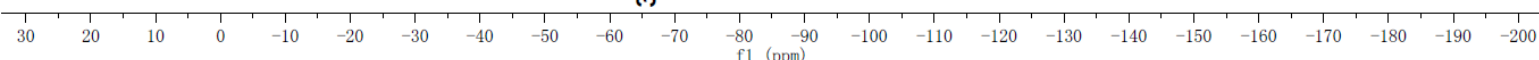

\section{3-(2, 2, 2-Trifluoroethyl)quinolone (26)}

2014614-6-101-H_PROTON_01
test001

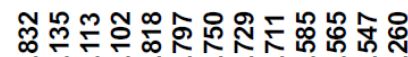

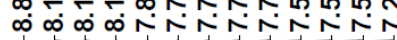

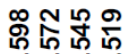

लं ले

ֻับ

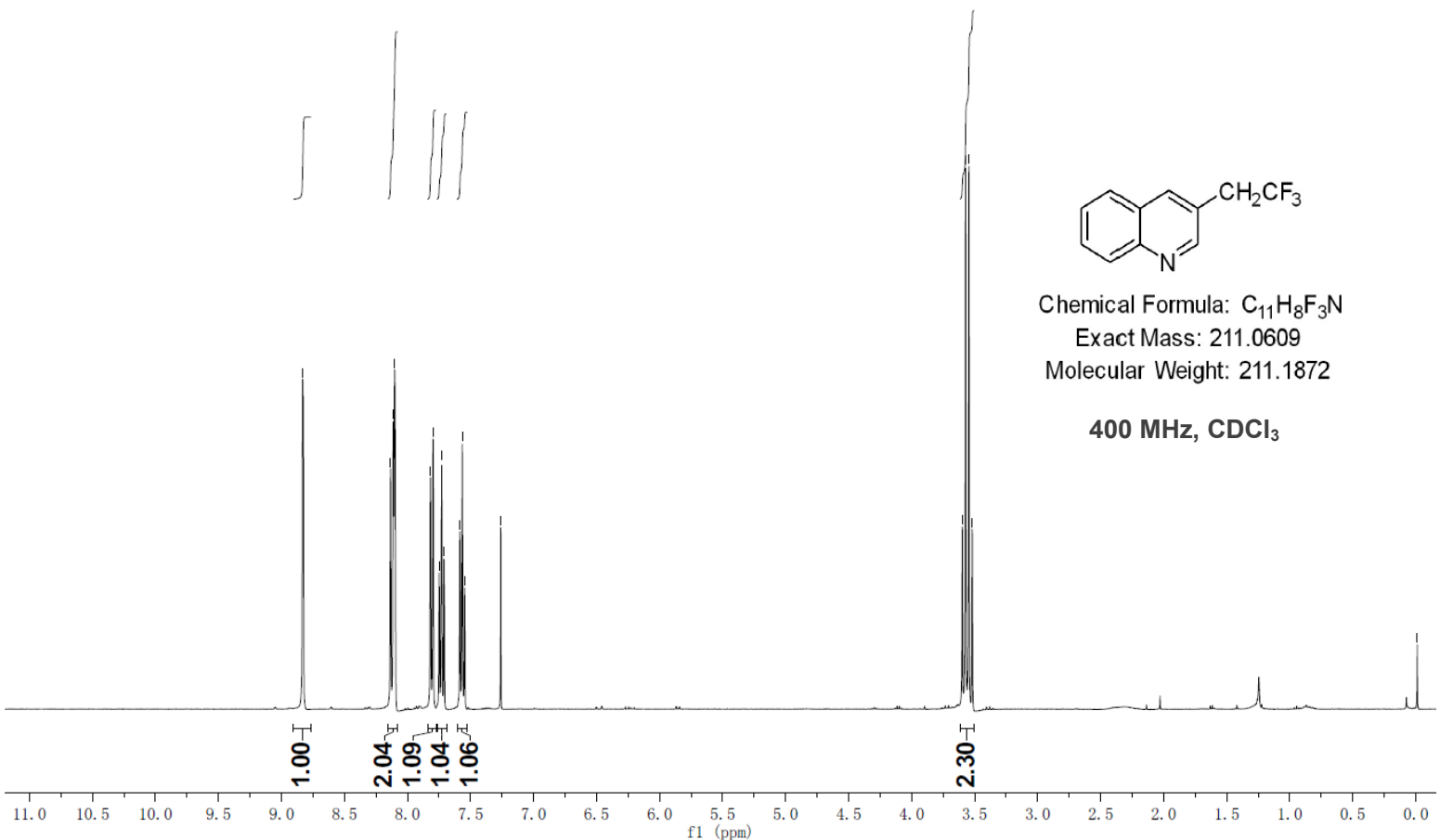


2014614-6-101C_pslabe1_01 test
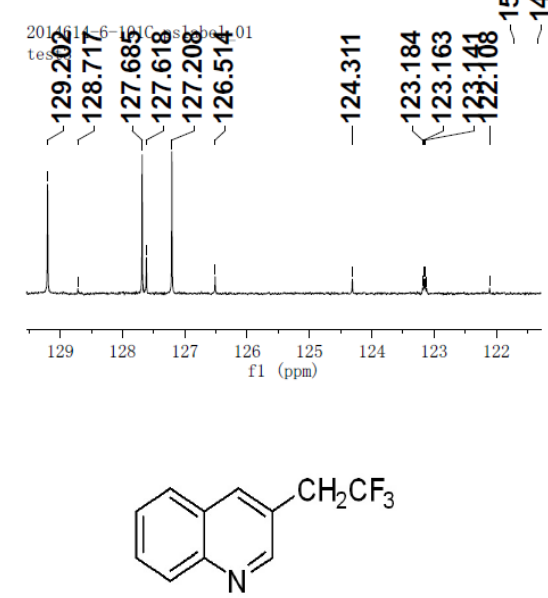

Chemical Formula: $\mathrm{C}_{11} \mathrm{H}_{8} \mathrm{~F}_{3} \mathrm{~N}$

Exact Mass: 211.0609

Molecular Weight: 211.1872

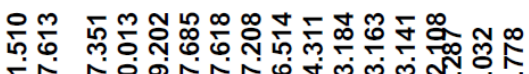

它守

유요용

क्लिलिल

$126 \mathrm{MHz}, \mathrm{CDCl}_{3}$

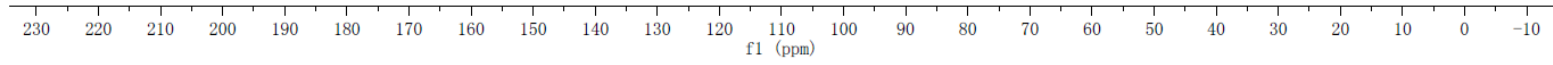

2014614-6-101-HF_FLUORINE_01

test001

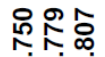

它它它

2014614-6-101-HF_FLUORINE test001

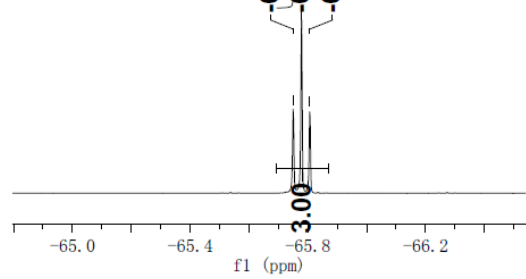

$376 \mathrm{MHz}, \mathrm{CDCl}_{3}$<smiles>FC(F)(F)Cc1cnc2ccccc2c1</smiles>

Chemical Formula: $\mathrm{C}_{11} \mathrm{H}_{8} \mathrm{~F}_{3} \mathrm{~N}$

Exact Mass: 211.0609

Molecular Weight: 211.1872

\section{8}

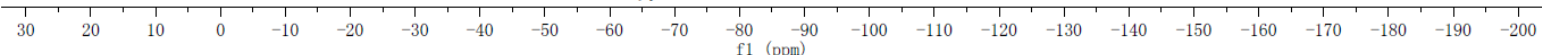


2-Methyl-6-(2, 2, 2-trifluoroethyl)quinolone (27)
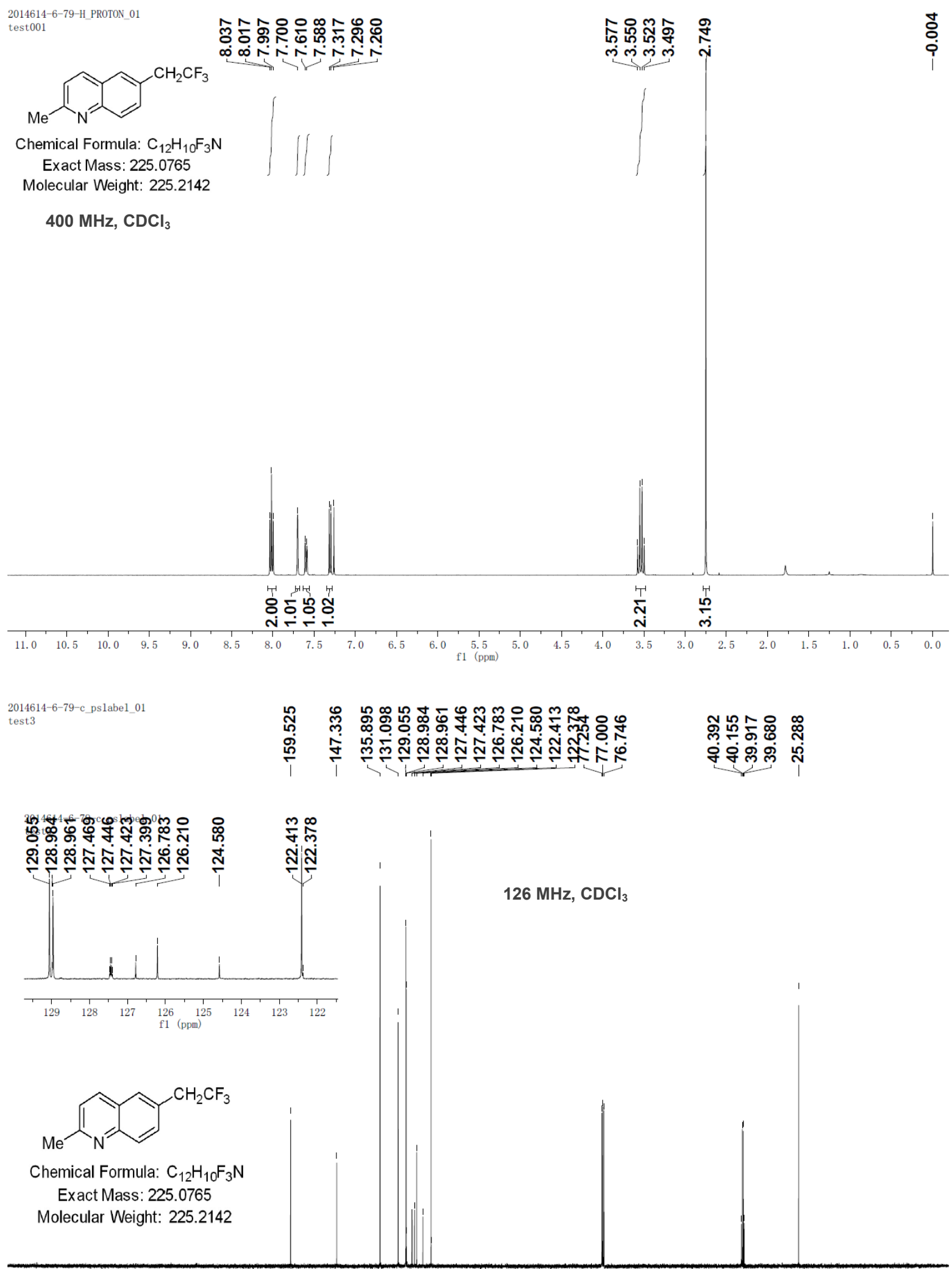

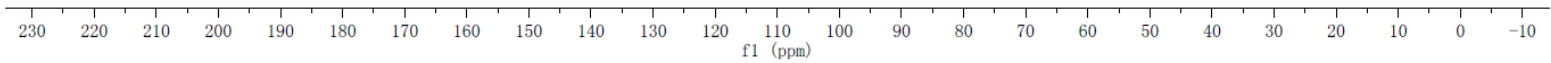




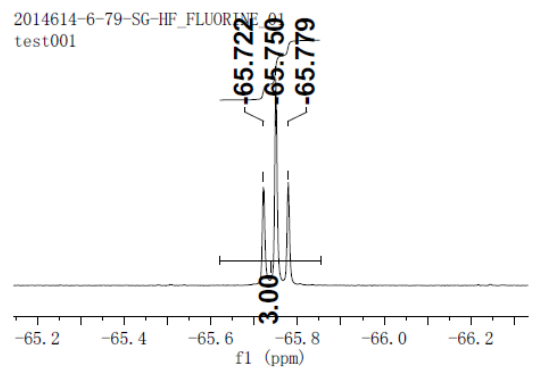

$376 \mathrm{MHz}, \mathrm{CDCl}_{3}$

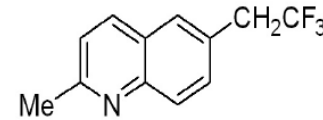

Chemical Formula: $\mathrm{C}_{12} \mathrm{H}_{10} \mathrm{~F}_{3} \mathrm{~N}$

Exact Mass: 225.0765

Molecular Weight: 225.2142

\section{$\stackrel{1}{8}$}

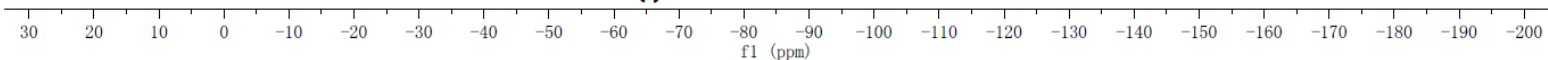

\section{7-(2, 2, 2-Trifluoroethyl)quinoline (28)}

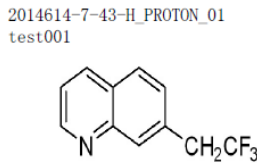

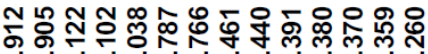

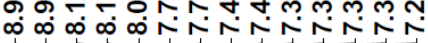

Chemical Formula: $\mathrm{C}_{11} \mathrm{H}_{8} \mathrm{~F}_{3} \mathrm{~N}$

Exact Mass: 211.0609

Molecular Weight: 211.1872

$400 \mathrm{MHz}^{\mathrm{CDCl}_{3}}$
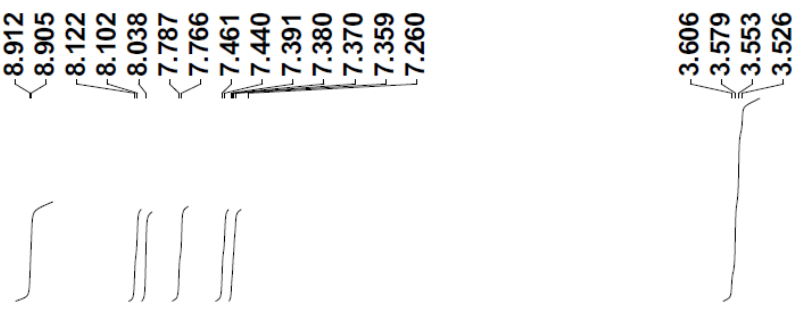

$\stackrel{\infty}{\circ}$

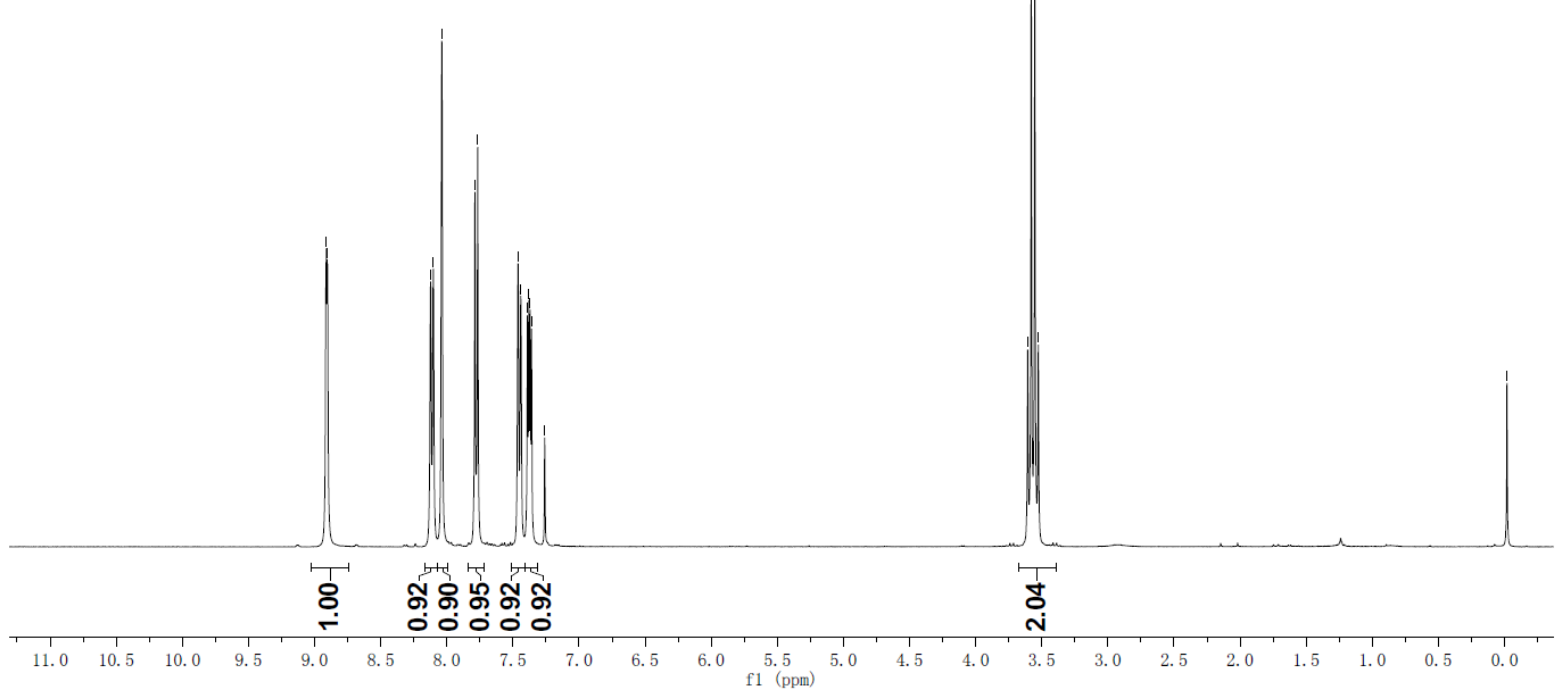




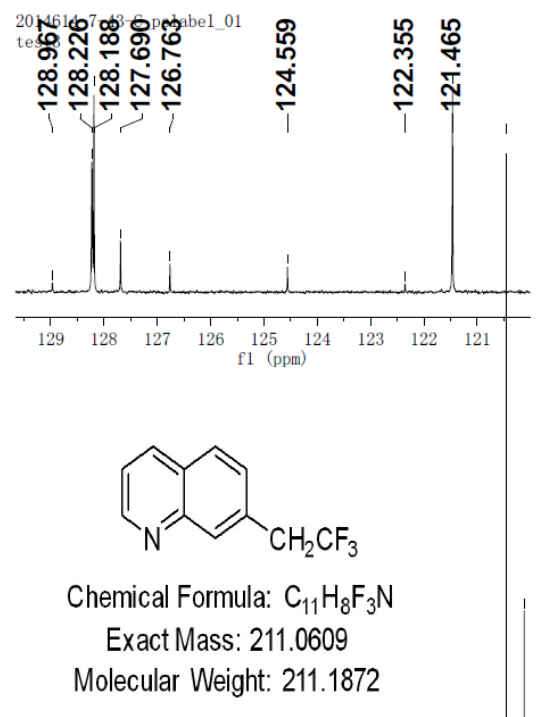

$126 \mathrm{MHz}, \mathrm{CDCl}_{3}$

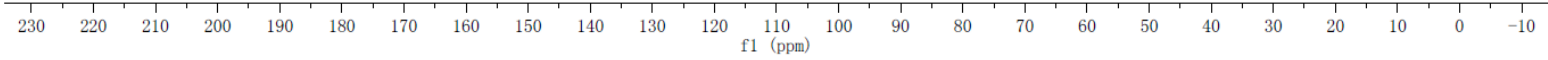

2014614-7-43-HF_FLUORINE_01 test001

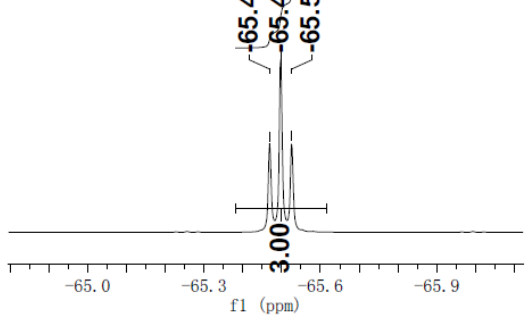

$376 \mathrm{MHz}, \mathrm{CDCl}_{3}$

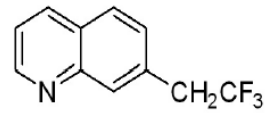

Chemical Formula: $\mathrm{C}_{11} \mathrm{H}_{8} \mathrm{~F}_{3} \mathrm{~N}$

Exact Mass: 211.0609

Molecular Weight: 211.1872

\section{8}

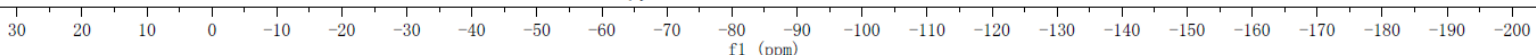


4-(2, 2, 2-Trifluoroethyl)isoquinoline (29)
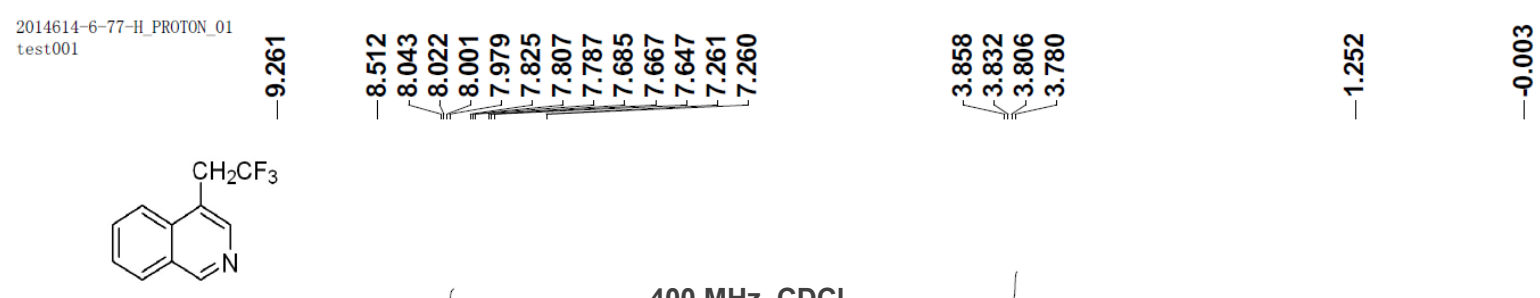

Chemical Formula: $\mathrm{C}_{11} \mathrm{H}_{8} \mathrm{~F}_{3} \mathrm{~N}$ Exact Mass: 211.0609

Molecular Weight: 211.1872
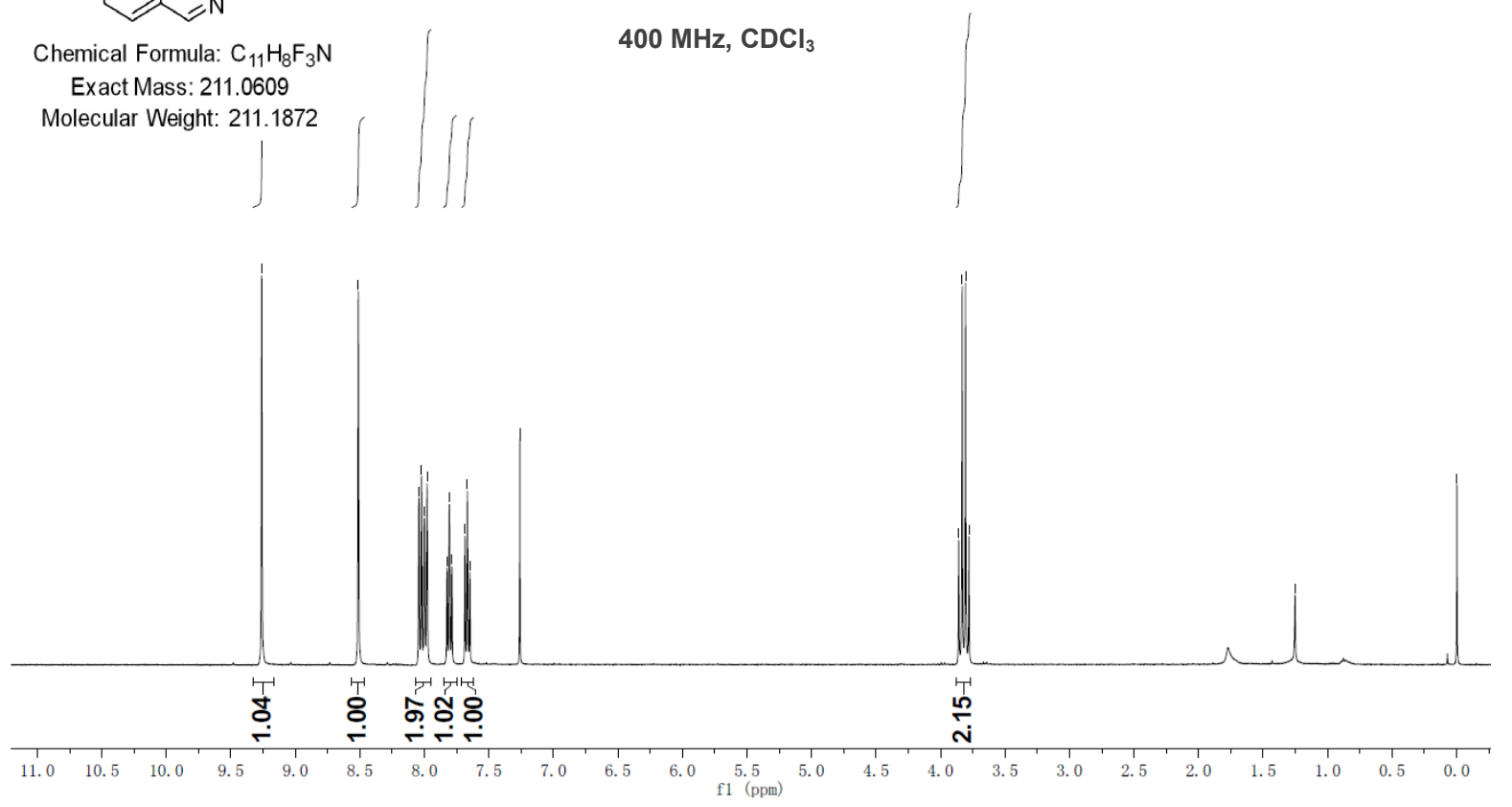

2014614-6-77-c_pslabel_01

test3

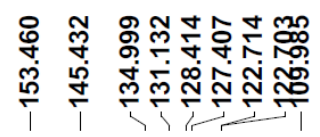

ํํํํํำ

余定

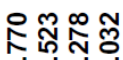

में

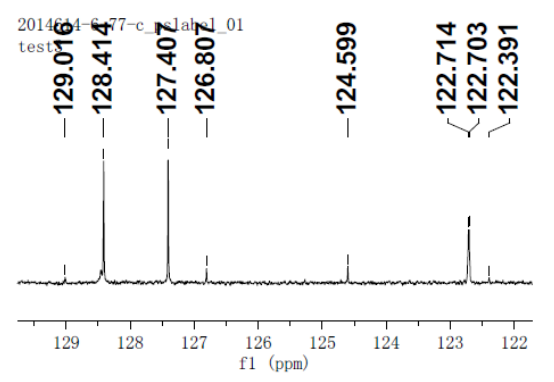

$126 \mathrm{MHz}, \mathrm{CDCl}_{3}$

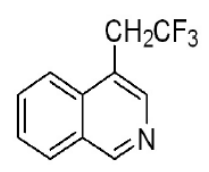

Chemical Formula: $\mathrm{C}_{11} \mathrm{H}_{8} \mathrm{~F}_{3} \mathrm{~N}$

Exact Mass: 211.0609

Molecular Weight: 211.1872

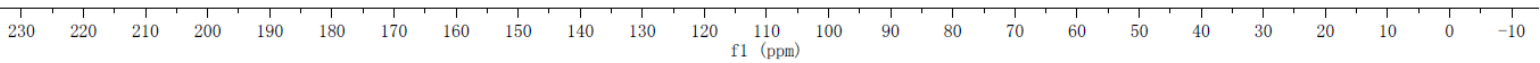


2014614-6-77-SG2-HF_FLUORINE_01

test001
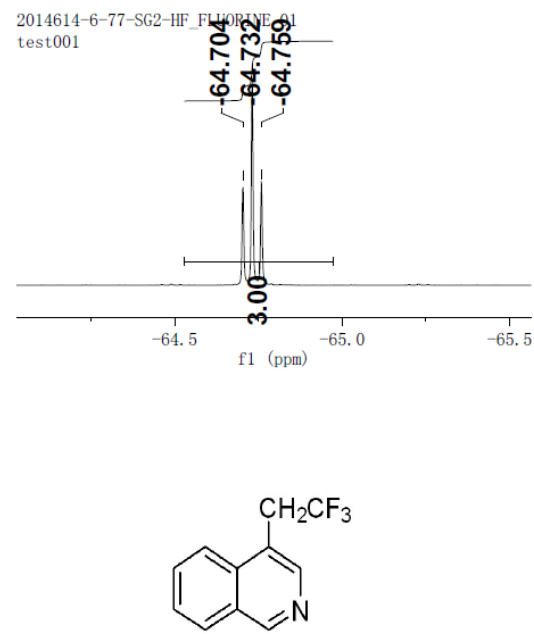

Chemical Formula: $\mathrm{C}_{11} \mathrm{H}_{8} \mathrm{~F}_{3} \mathrm{~N}$

Exact Mass: 211.0609

Molecular Weight: 211.1872

\section{호유요}

फ़்

$376 \mathrm{MHz}, \mathrm{CDCl}_{3}$

\section{웅}

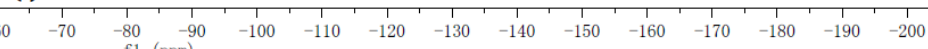

\section{6-(2, 2, 2-Trifluoroethyl)isoquinoline (30)}
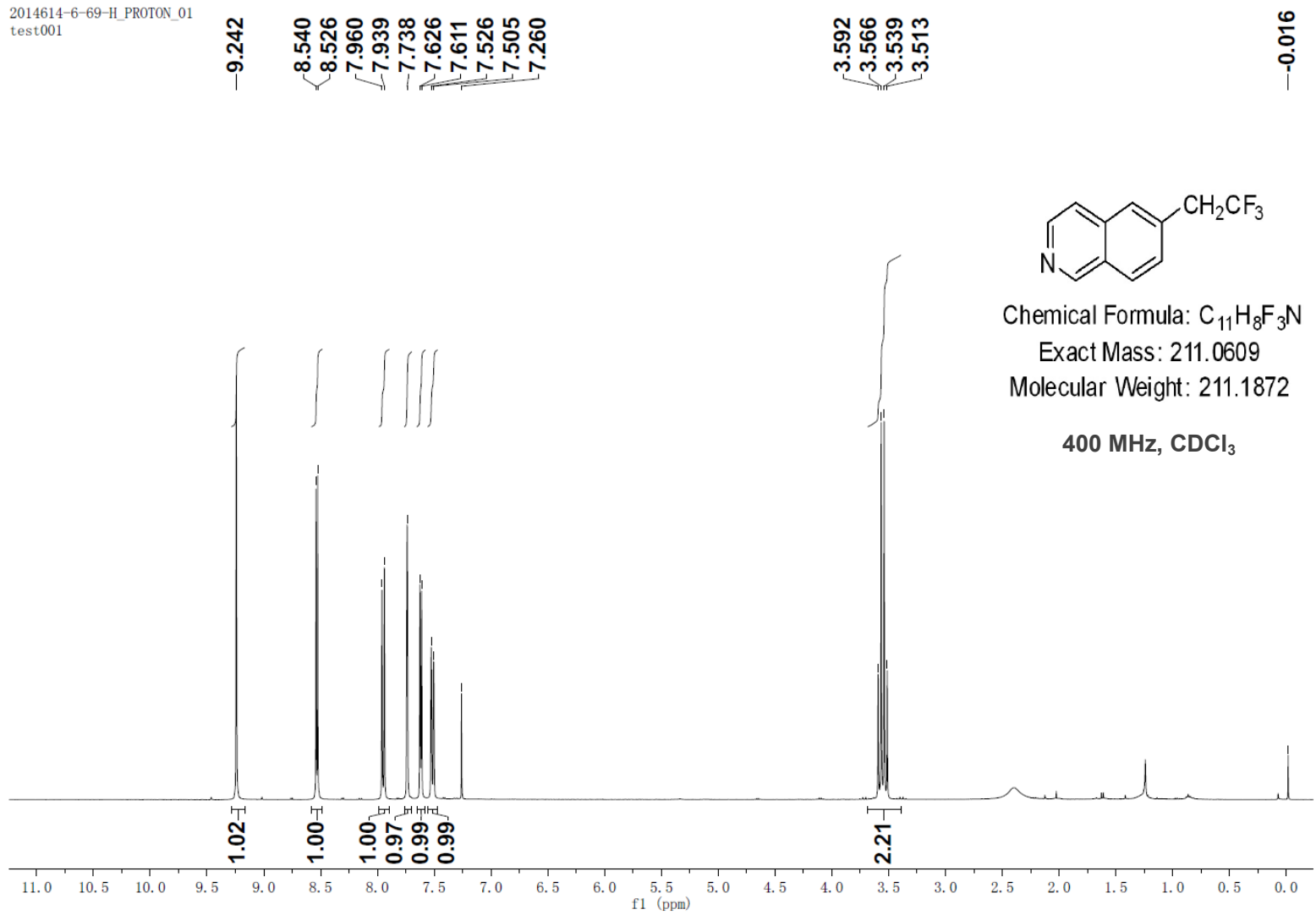

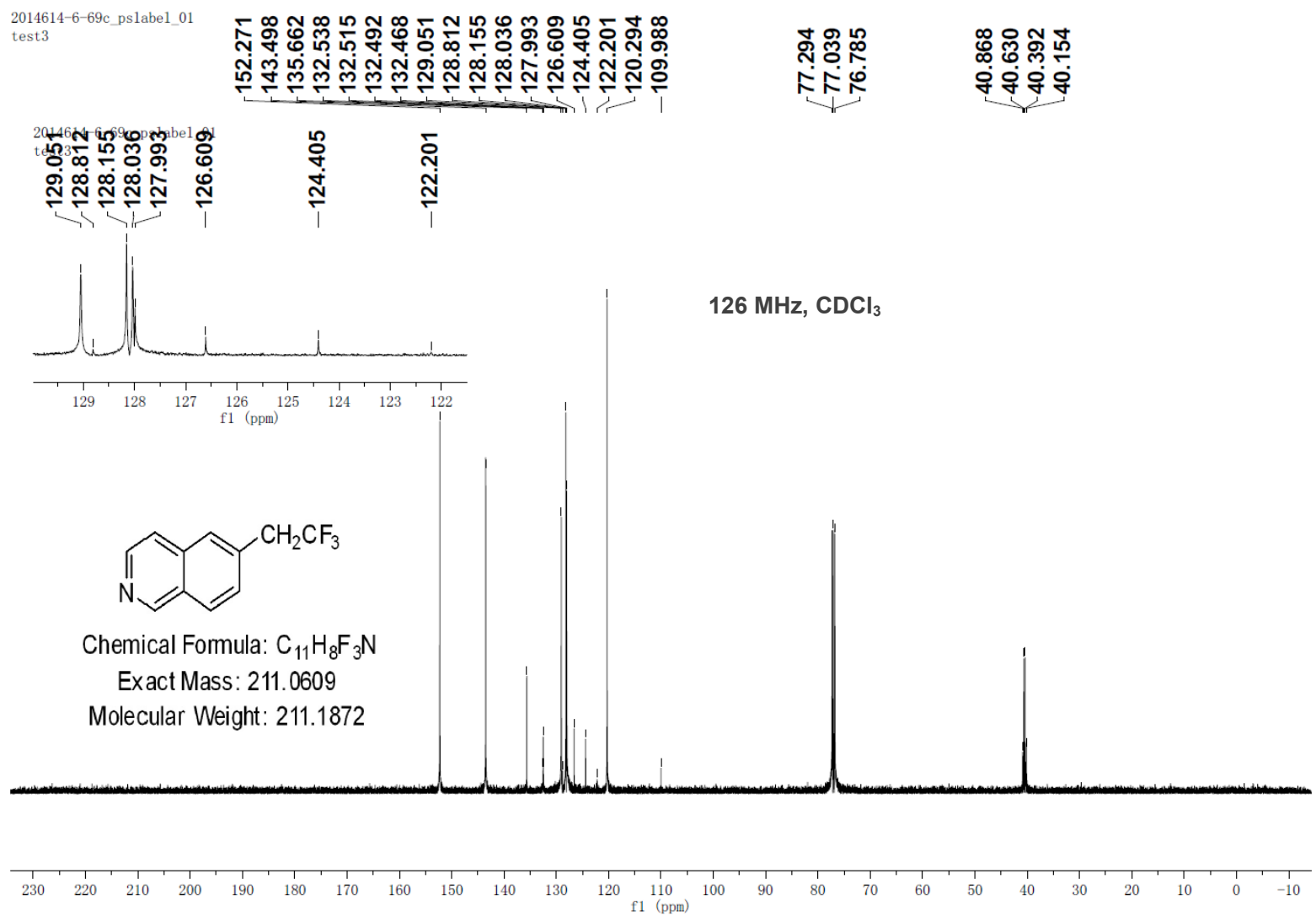

2014614-6-69-HF_FLUORINE_01

test 00

았 용영

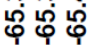

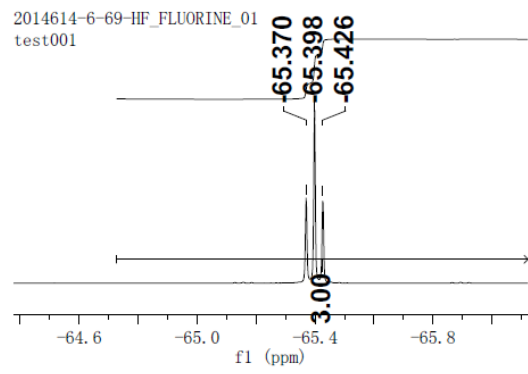

$376 \mathrm{MHz}, \mathrm{CDCl}_{3}$

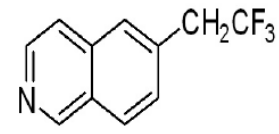

Chemical Formula: $\mathrm{C}_{11} \mathrm{H}_{8} \mathrm{~F}_{3} \mathrm{~N}$

Exact Mass: 211.0609

Molecular Weight: 211.1872

\section{$\stackrel{4}{8}$}

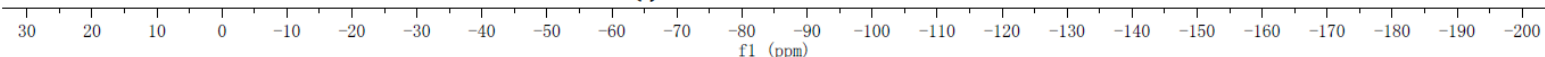


Tert-Butyl 4-(2, 2, 2-trifluoroethyl)-1H-indole-1-carboxylate (31)

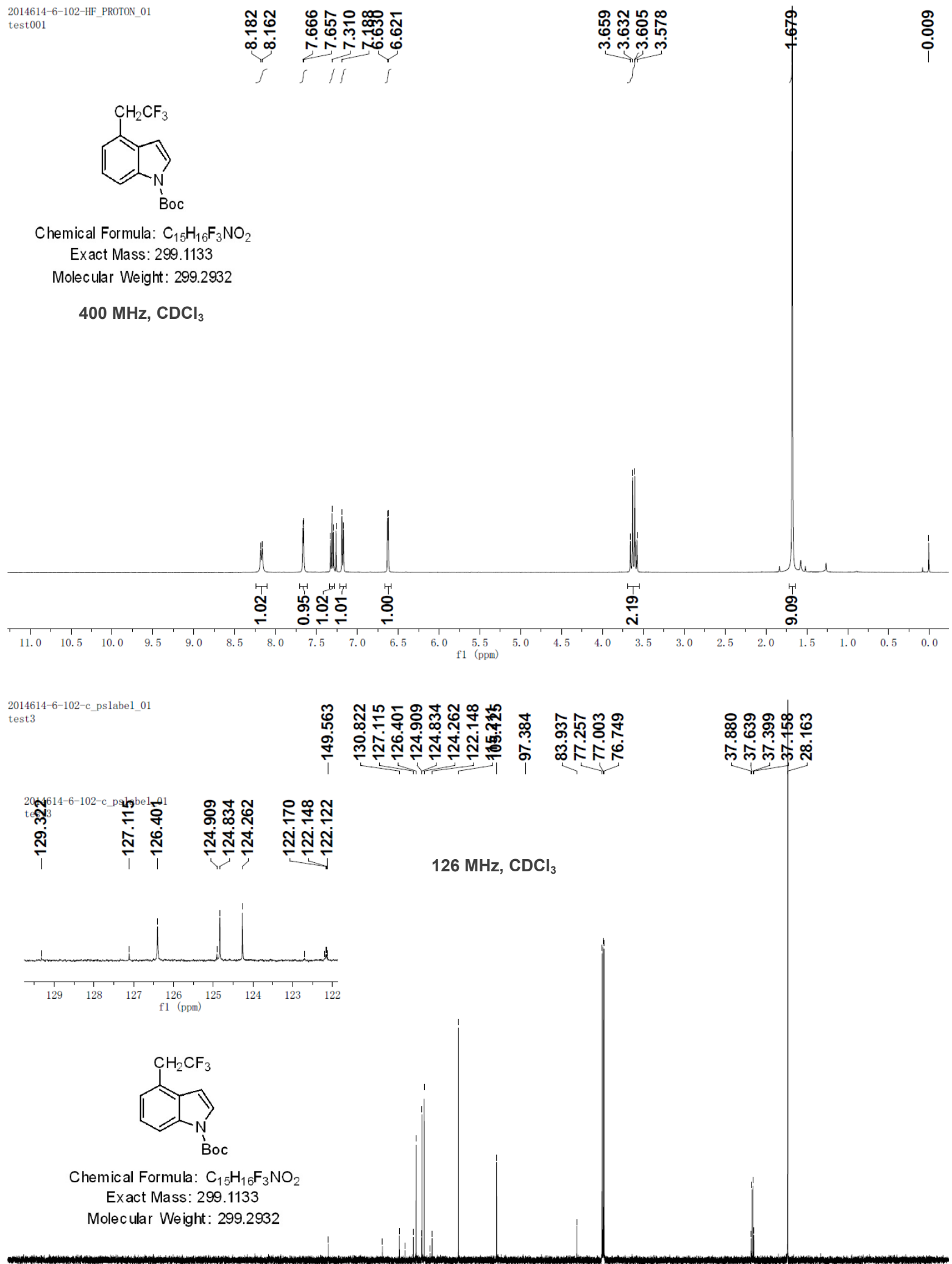

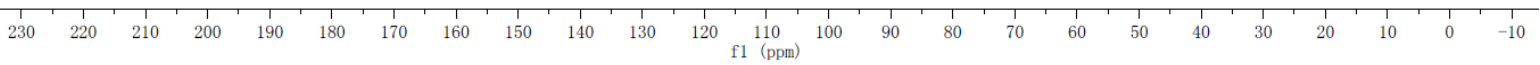




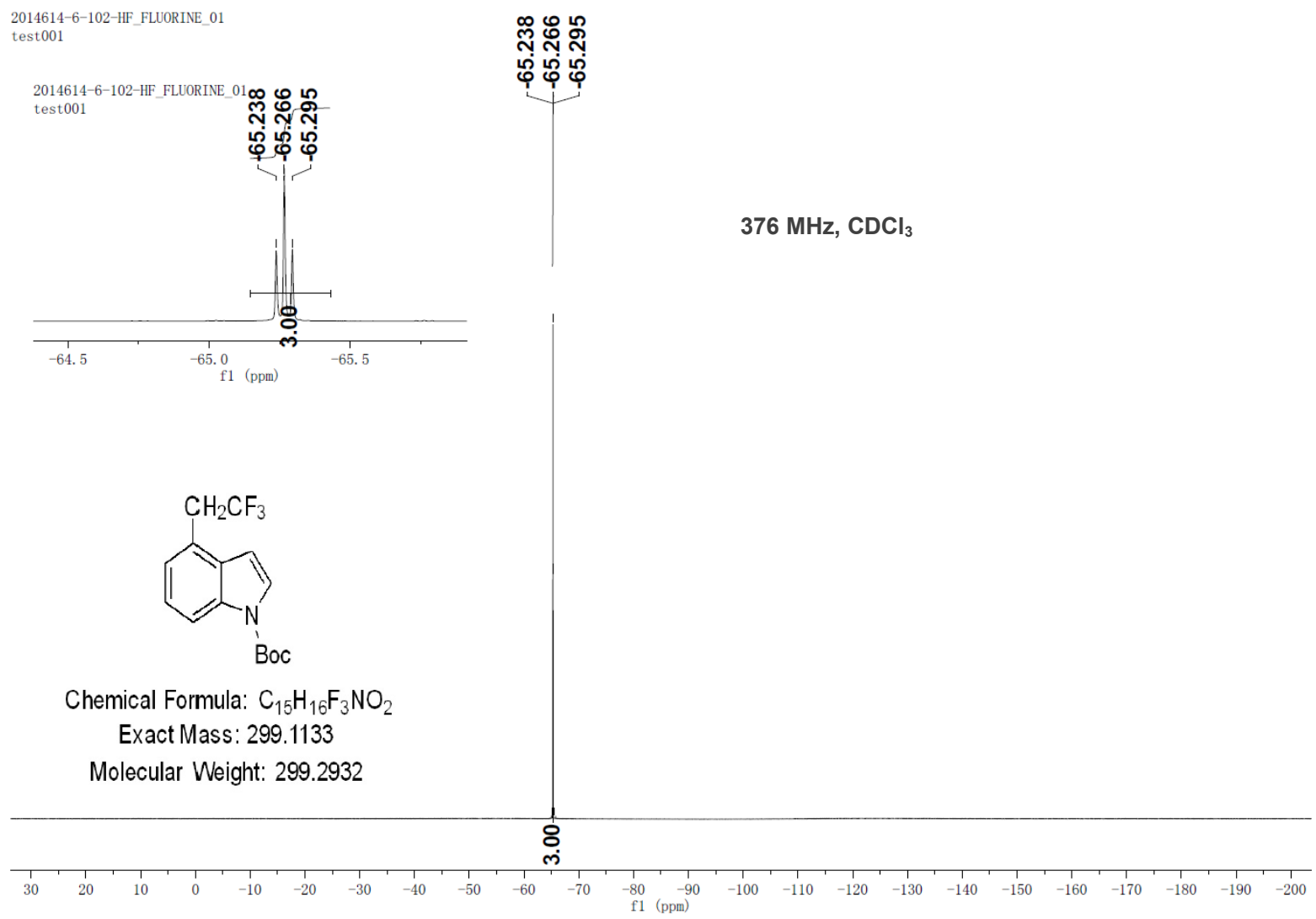

1-(Tert-butyldimethylsilyl)-4-(2,2,2-trifluoroethyl)-1H-indole (32)

2014614-7-44-HF_PROTON_0
test001

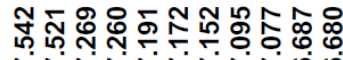

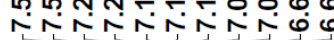

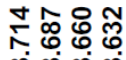
ஸ் लं ले<smiles>CC(C)(C)[Si](C)(C)n1ccc2c(CC(F)(F)F)cccc21</smiles>

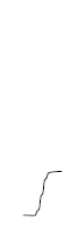

Chemical Formula: $\mathrm{C}_{16} \mathrm{H}_{22} \mathrm{~F}_{3} \mathrm{NSi}$

Exact Mass: 313.1474

Molecular Weight: 313.4392

$400 \mathrm{MHz}, \mathrm{CDCl}_{3}$
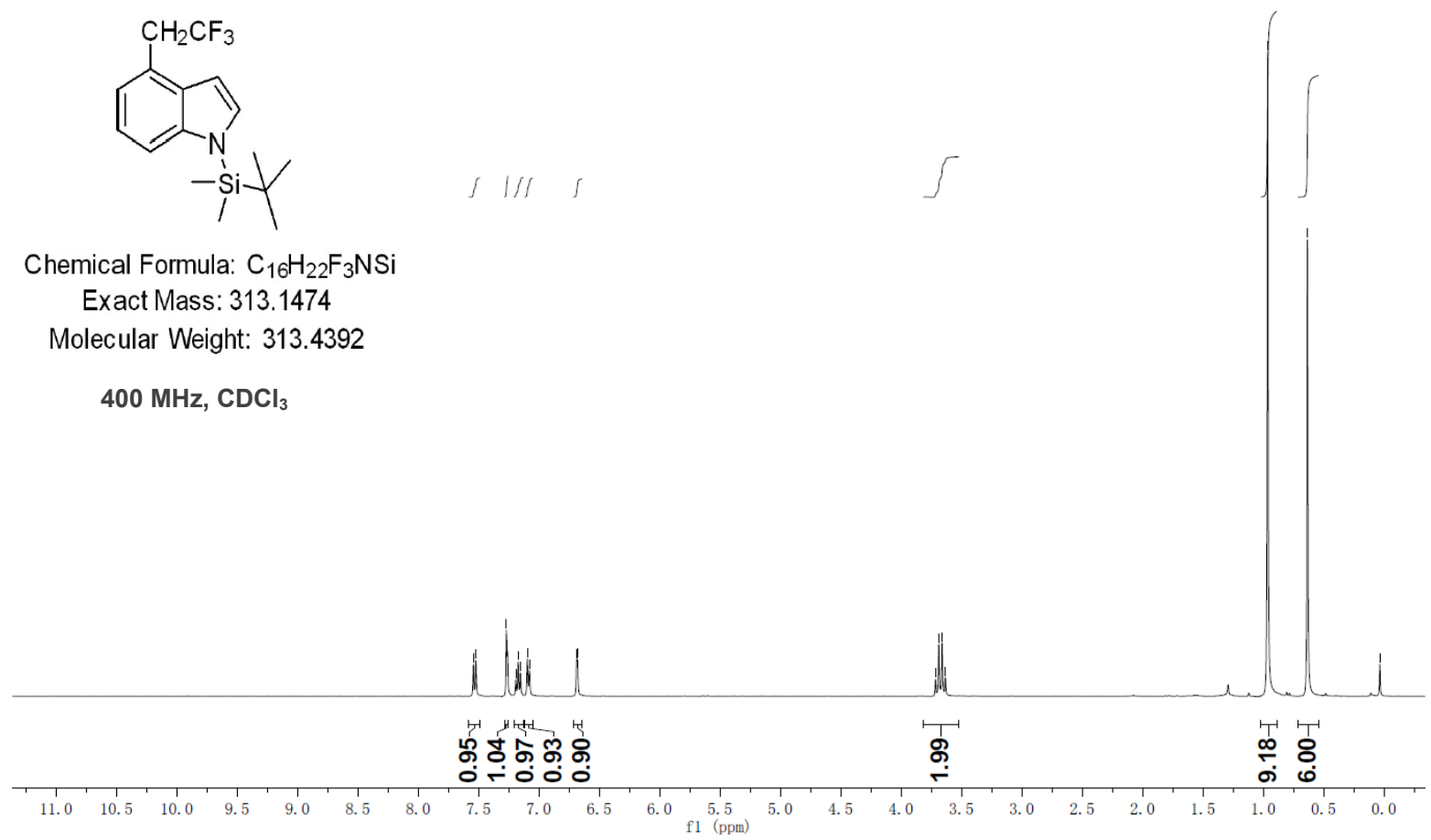
2014614-7-44-C_pslabe1_01

2014614-7-44-C_pslabel_01
test3

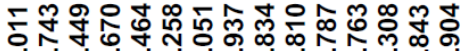

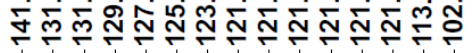

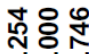

ㅅํㅇ

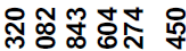

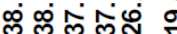

ำ लूलिक ?

$126 \mathrm{MHz}, \mathrm{CDCl}_{3}$
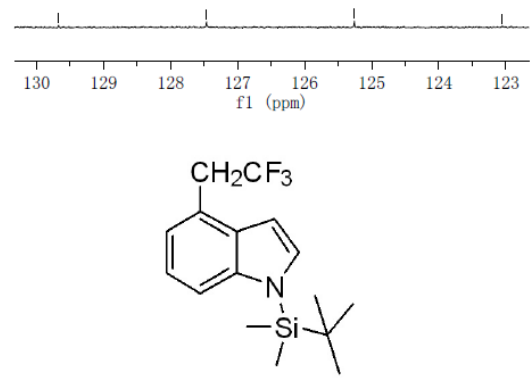

Chemical Formula: $\mathrm{C}_{16} \mathrm{H}_{22} \mathrm{~F}_{3} \mathrm{NSi}$

Exact Mass: 313.1474

Molecular Weight: 313.4392

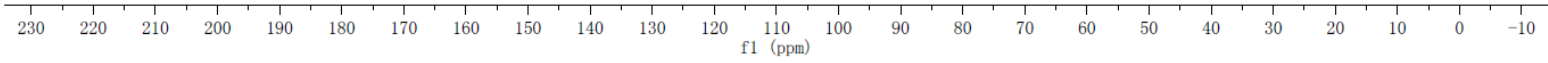

2014614-7-44-HF_FLUORINE_01 test001

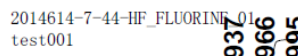

ড़广

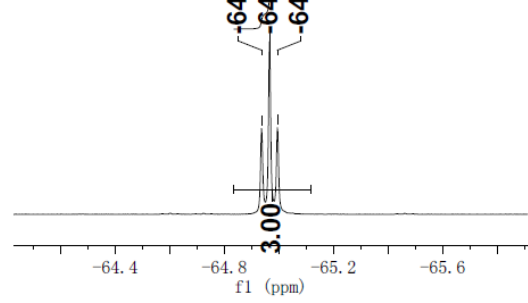

$376 \mathrm{MHz}, \mathrm{CDCl}_{3}$

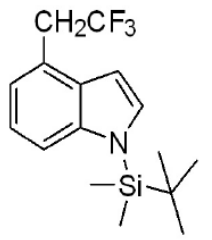

Chemical Formula: $\mathrm{C}_{16} \mathrm{H}_{22} \mathrm{~F}_{3} \mathrm{NSi}$

Exact Mass: 313.1474

Molecular Weight: 313.4392

\section{8}

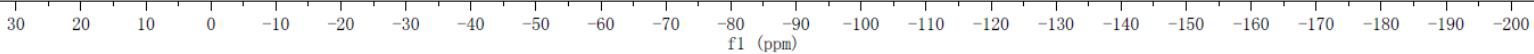



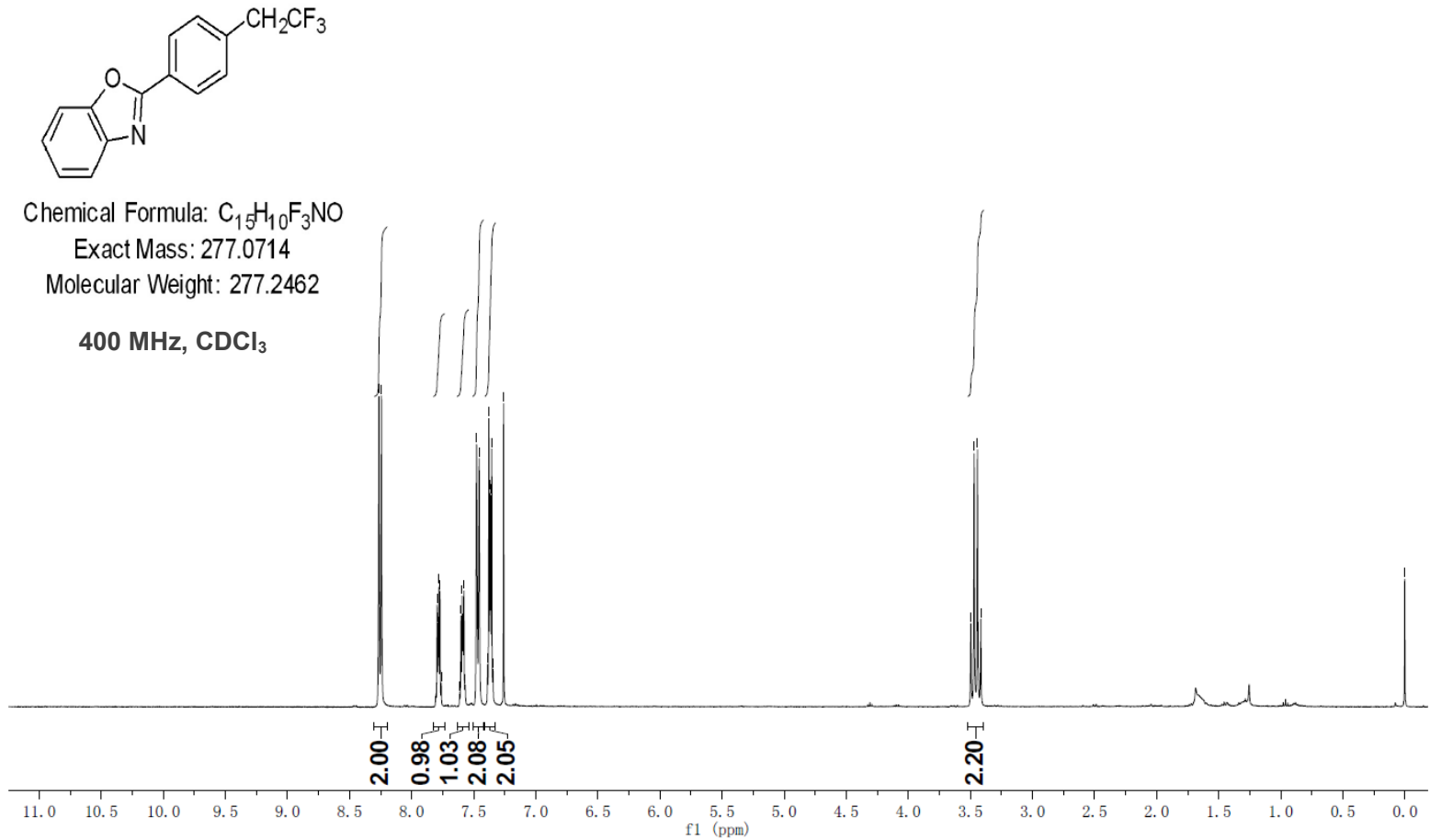

2014614-7-15c_pslabe1_01 test3

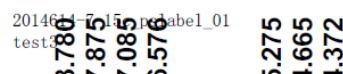

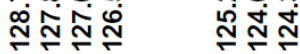
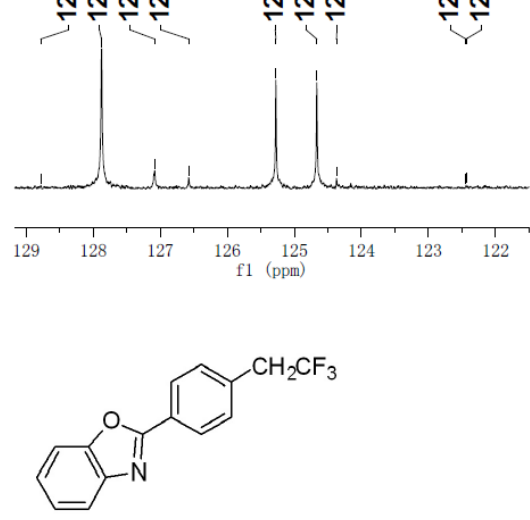

Chemical Formula: $\mathrm{C}_{15} \mathrm{H}_{10} \mathrm{~F}_{3} \mathrm{NO}$ Exact Mass: 277.0714 Molecular Weight: 277.2462
员 $\overline{0}$ 莳

ชิ

ษัฐ ชิ

บู

$126 \mathrm{MHz}^{\mathrm{CDCl}_{3}}$

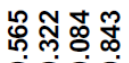

항ํㅇ 


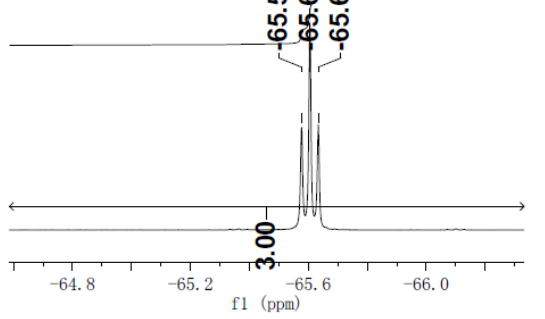

$376 \mathrm{MHz}, \mathrm{CDCl}_{3}$

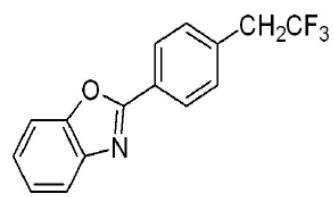

Chemical Formula: $\mathrm{C}_{15} \mathrm{H}_{10} \mathrm{~F}_{3} \mathrm{NO}$

Exact Mass: 277.0714

Molecular Weight: 277.2462

\section{8}

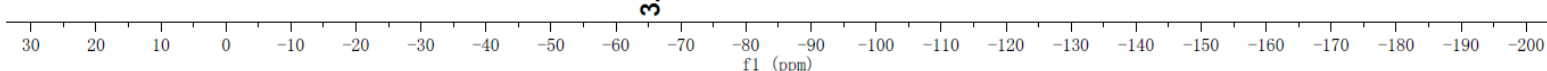

\section{Ethyl 2-methyl-2-(4-(2, 2, 2-trifluoroethyl)phenoxy)propanoate (35a)}

2014614-7-74-HF_PROTON_01 test001<smiles>CCOC(=O)C(C)(C)Oc1ccc(CC(F)F)cc1</smiles>

Chemical Formula: $\mathrm{C}_{14} \mathrm{H}_{17} \mathrm{~F}_{3} \mathrm{O}_{3}$ Exact Mass: 290.1130

Molecular Weight: 290.2822 $400 \mathrm{MHz}, \mathrm{CDCl}_{3}$
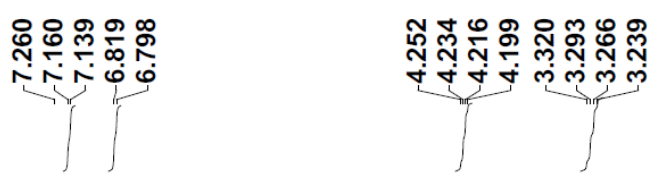

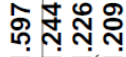




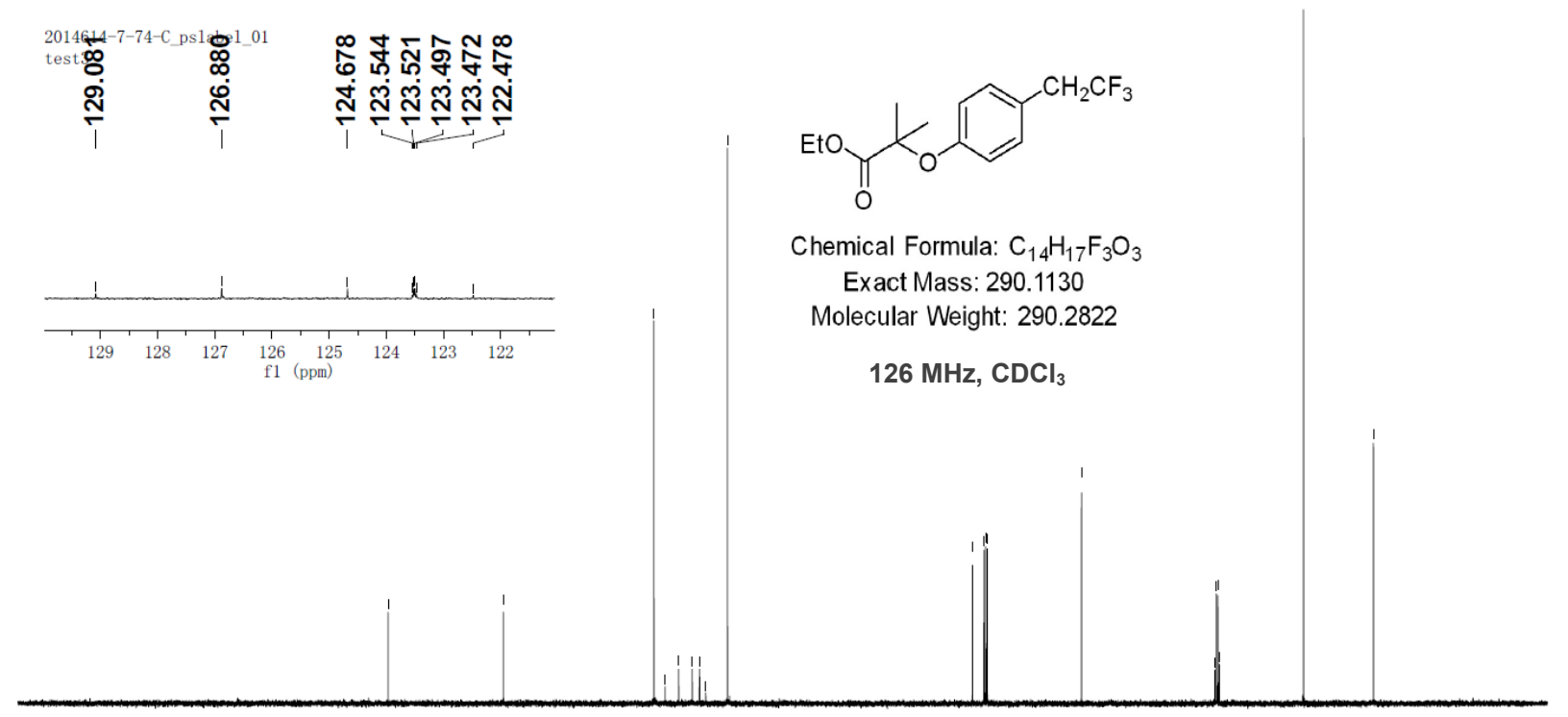

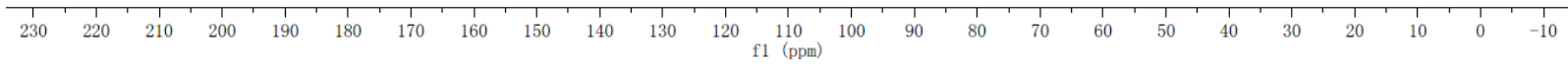

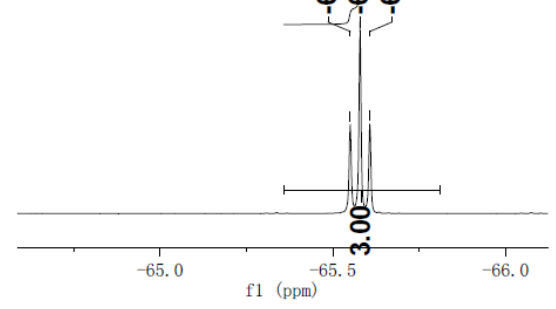

$376 \mathrm{MHz}, \mathrm{CDCl}_{3}$

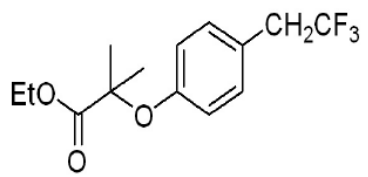

Chemical Formula: $\mathrm{C}_{14} \mathrm{H}_{17} \mathrm{~F}_{3} \mathrm{O}_{3}$

Exact Mass: 290.1130

Molecular Weight: 290.2822

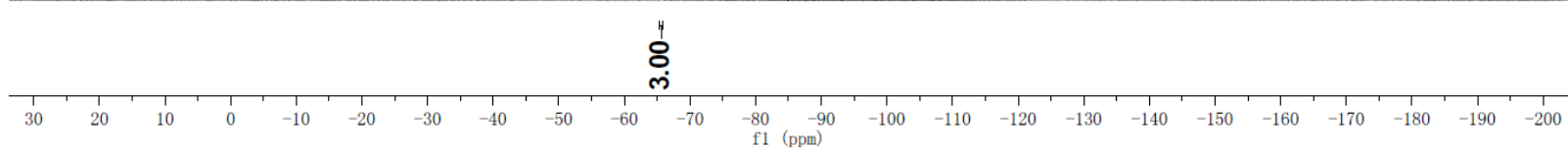


Isopropyl 2-methyl-2-(4-(4-(2, 2, 2-trifluoroethyl)benzoyl)phenoxy)propanoate (35b)
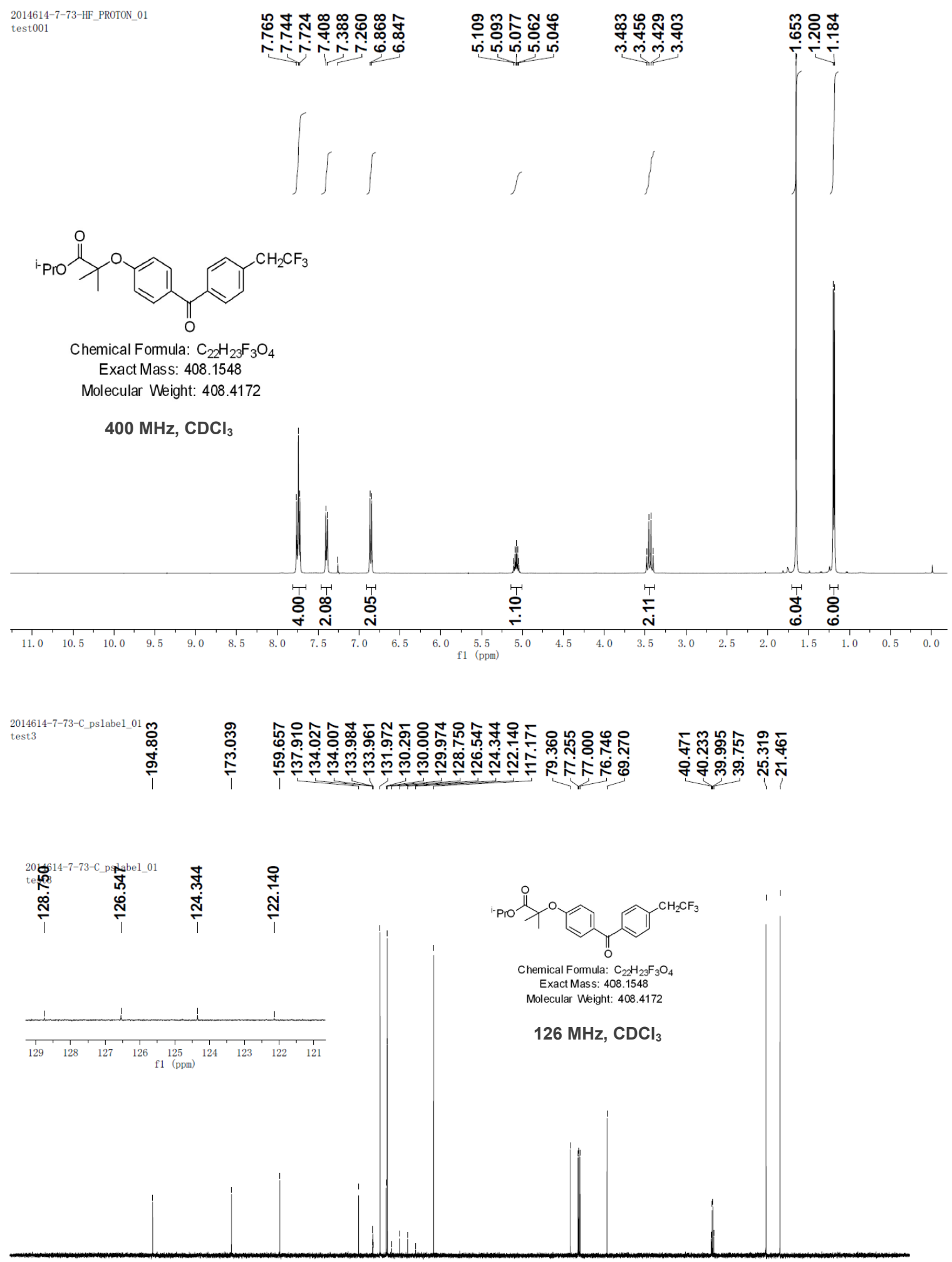

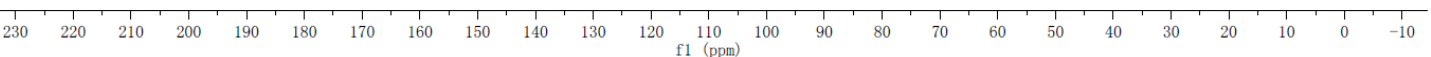


2014614-7-73-HF_FLUORINE_01

test001

2014614-7-73-HF_FLUORINE_0

test001

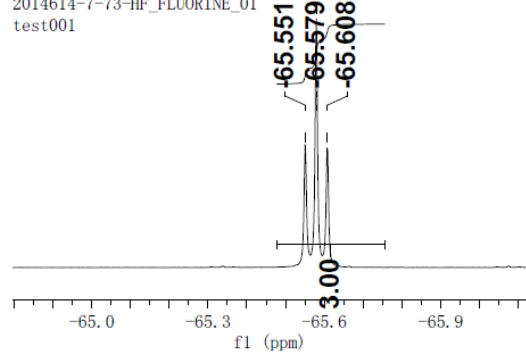

${ }^{\mathrm{P}} \mathrm{PrO} \mathrm{N}_{\mathrm{O}}^{\mathrm{O}}$

Chemical Formula: $\mathrm{C}_{22} \mathrm{H}_{23} \mathrm{~F}_{3} \mathrm{O}_{4}$

Exact Mass: 408.1548

Molecular Weight: 408.4172
둥ำ

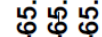

$376 \mathrm{MHz}, \mathrm{CDCl}_{3}$

1-Methyl-2-(4-(2, 2, 2-trifluoroethyl)phenyl)-1, 3-thiazinan-4-one 1, 1-dioxide (35c)

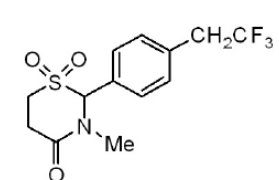

Chemical Formula: $\mathrm{C}_{13} \mathrm{H}_{14} \mathrm{~F}_{3} \mathrm{NO}_{3} \mathrm{~S}$

Exact Mass: 321.0646

Molecular Weight: 321.3142

$400 \mathrm{MHz}, \mathrm{CDCl}_{3}$
ल्ల్లో

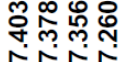

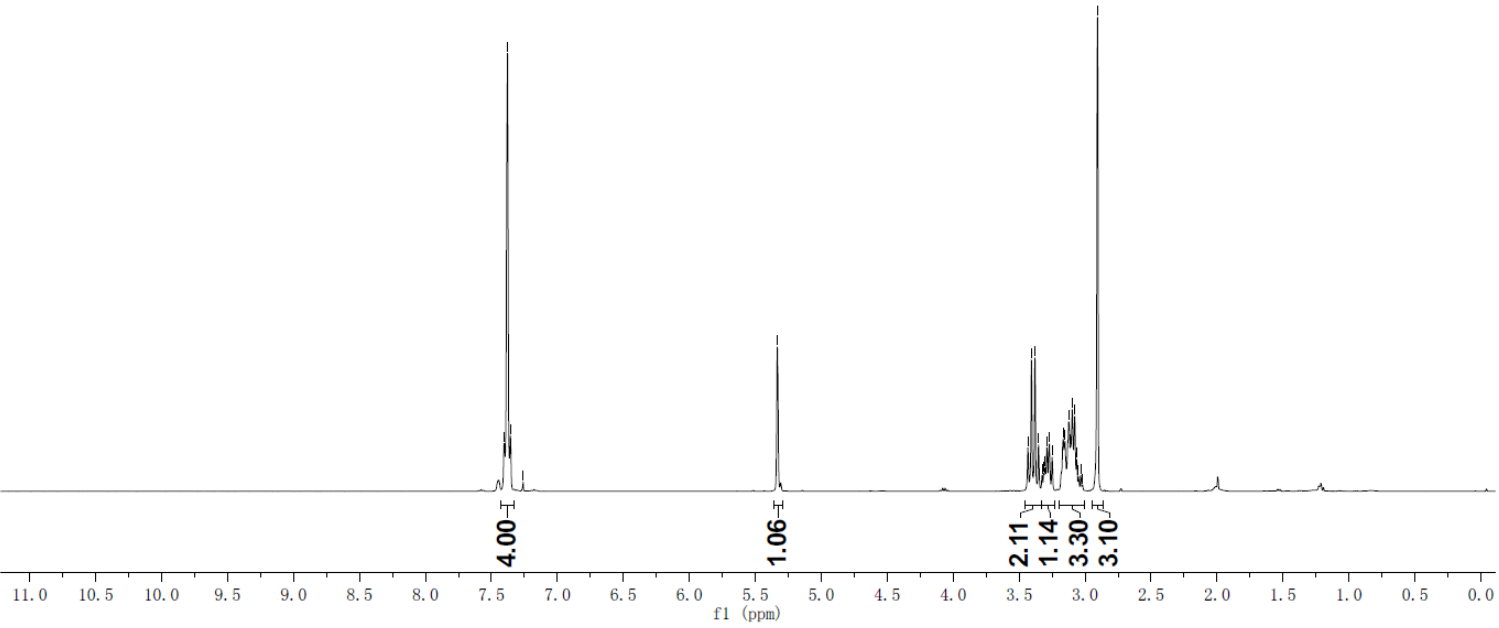




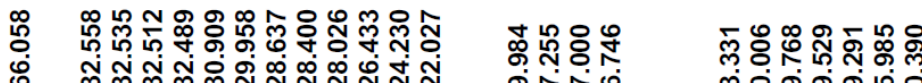

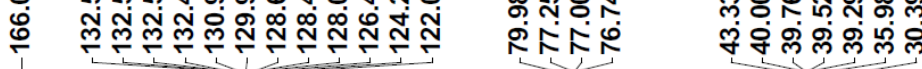
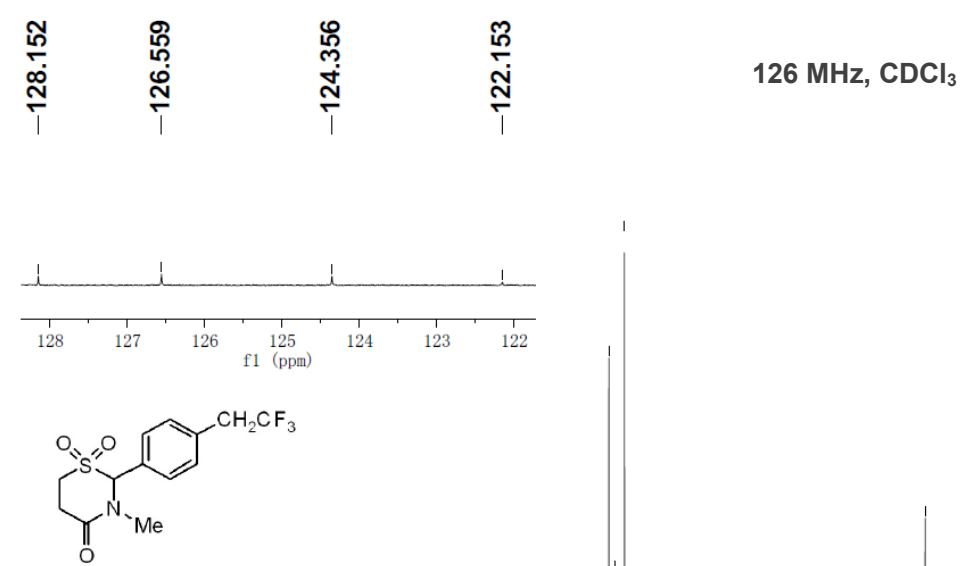

Chemical Formula: $\mathrm{C}_{13} \mathrm{H}_{14} \mathrm{~F}_{3} \mathrm{NO}_{3} \mathrm{~S}$

Exact Mass: 321.0646

Molecular Weight: 321.3142
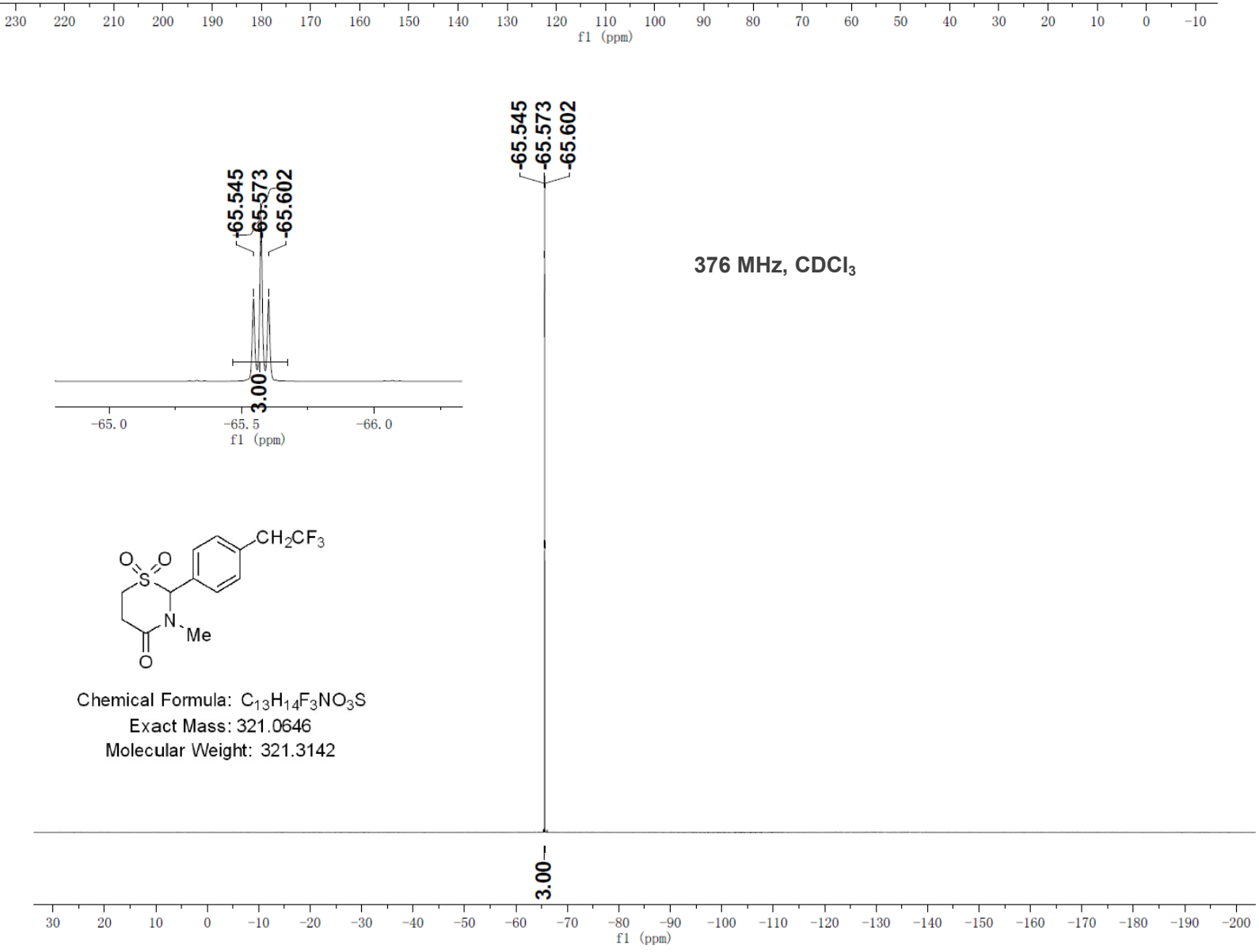
Methyl 2-(2-methyl-1-(4-(2, 2, 2-trifluoroethyl)benzoyl)-1H-indol-3-yl)acetate (35d)

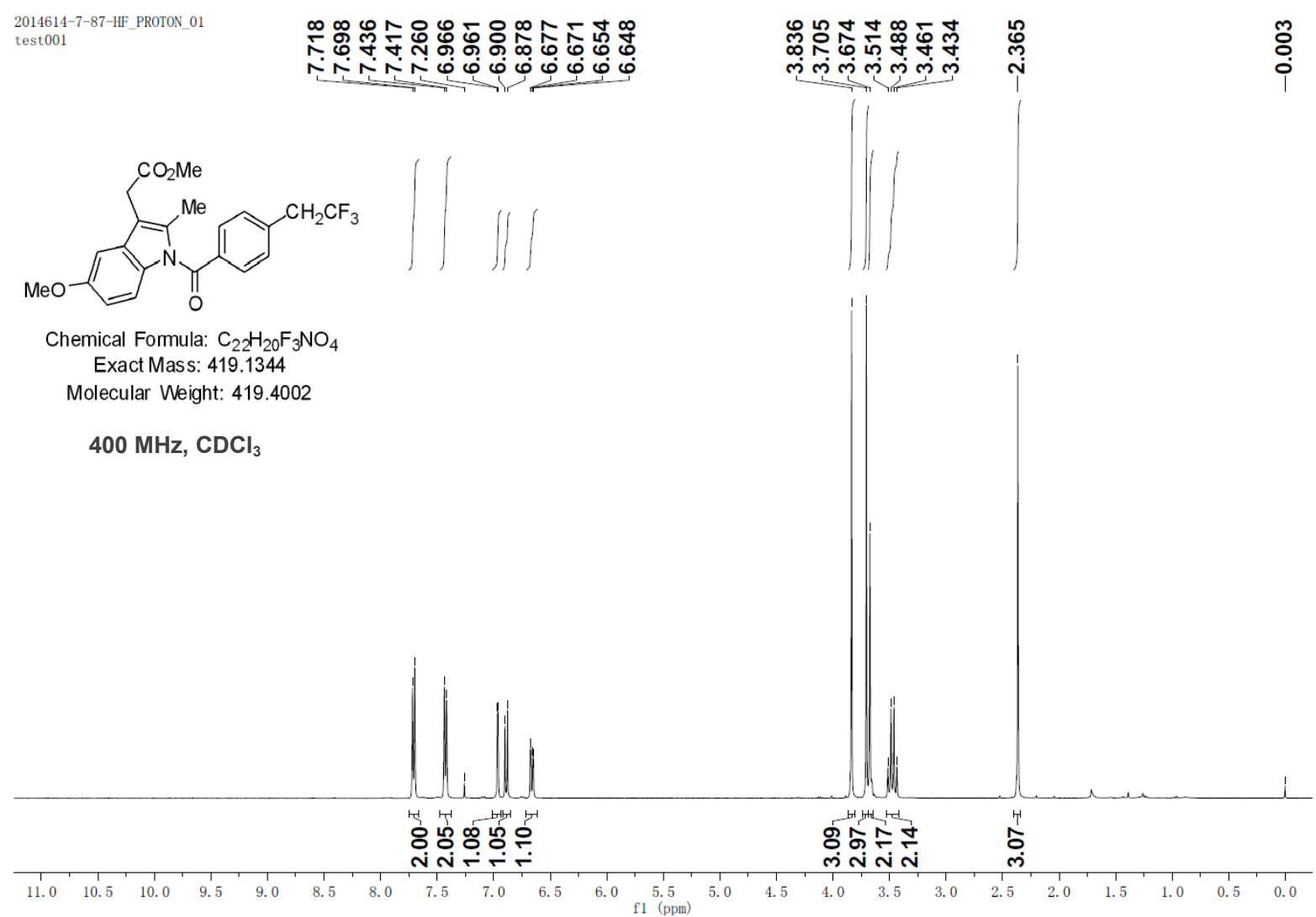

2014614-7-87-C_pslabel_01

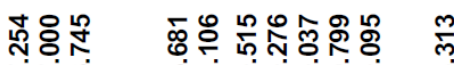

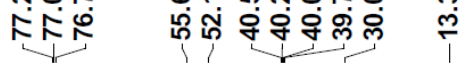

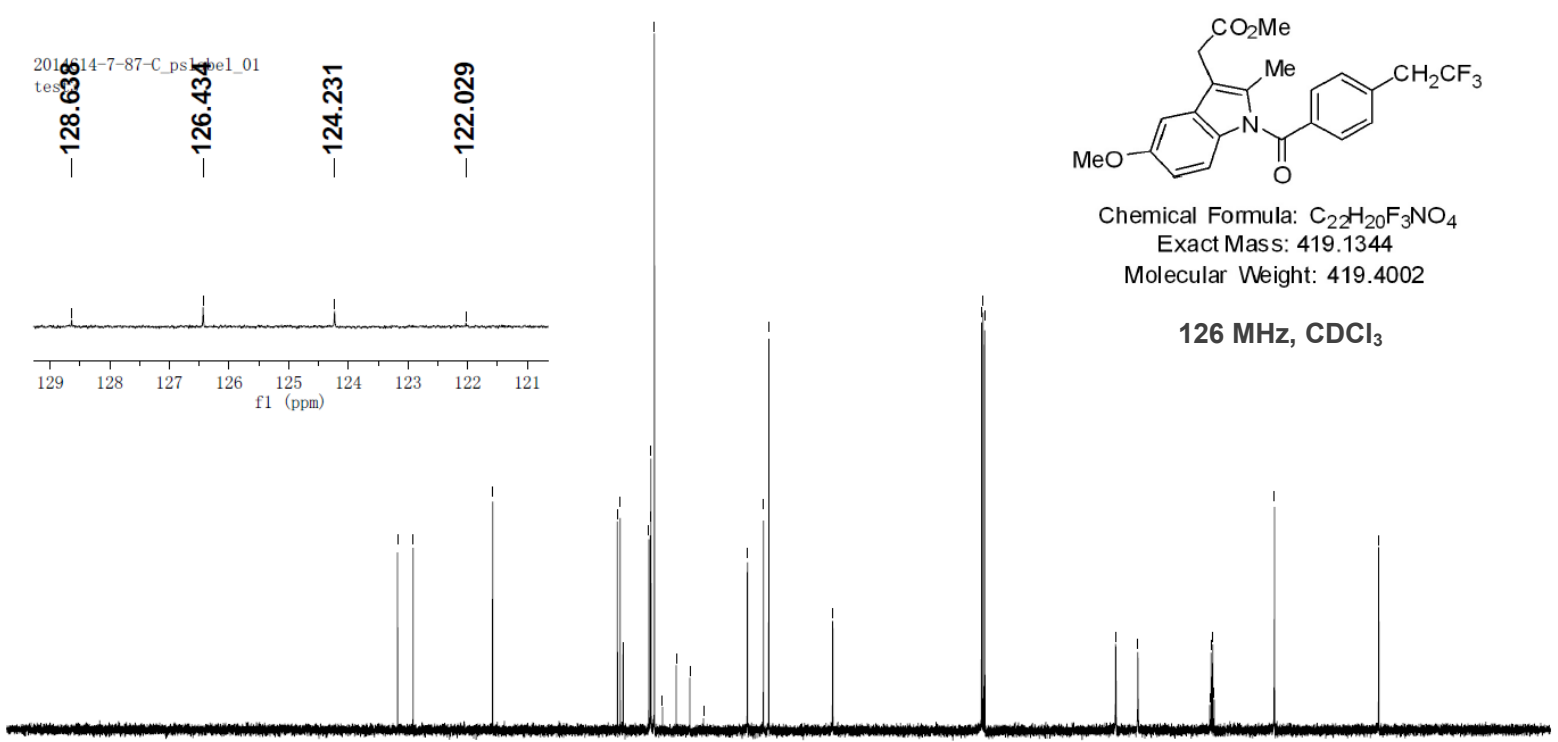

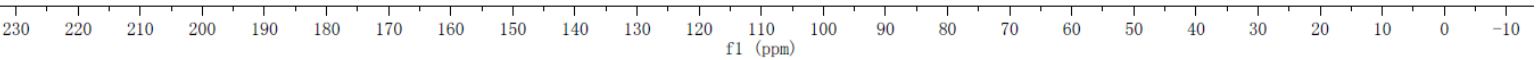




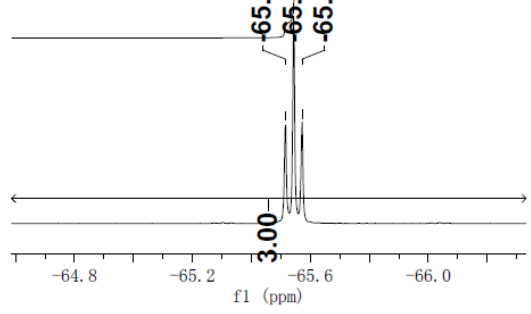

$376 \mathrm{MHz}, \mathrm{CDCl}_{3}$

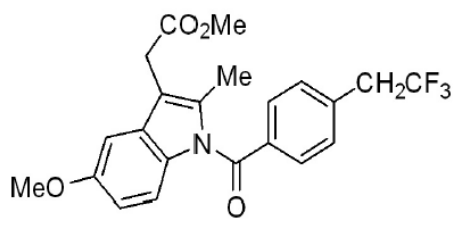

Chemical Formula: $\mathrm{C}_{22} \mathrm{H}_{20} \mathrm{~F}_{3} \mathrm{NO}_{4}$ Exact Mass: 419.1344 Molecular Weight: 419.4002

\section{8}

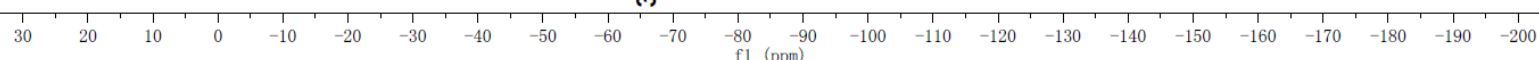

Ethyl 4-(8-(2, 2, 2-trifluoroethyl)-5, 6-dihydro-11H-benzo[5, 6]cyclohepta[1, 2-b]pyridin-11-ylidene)piperidine-1-carboxylate (35e)

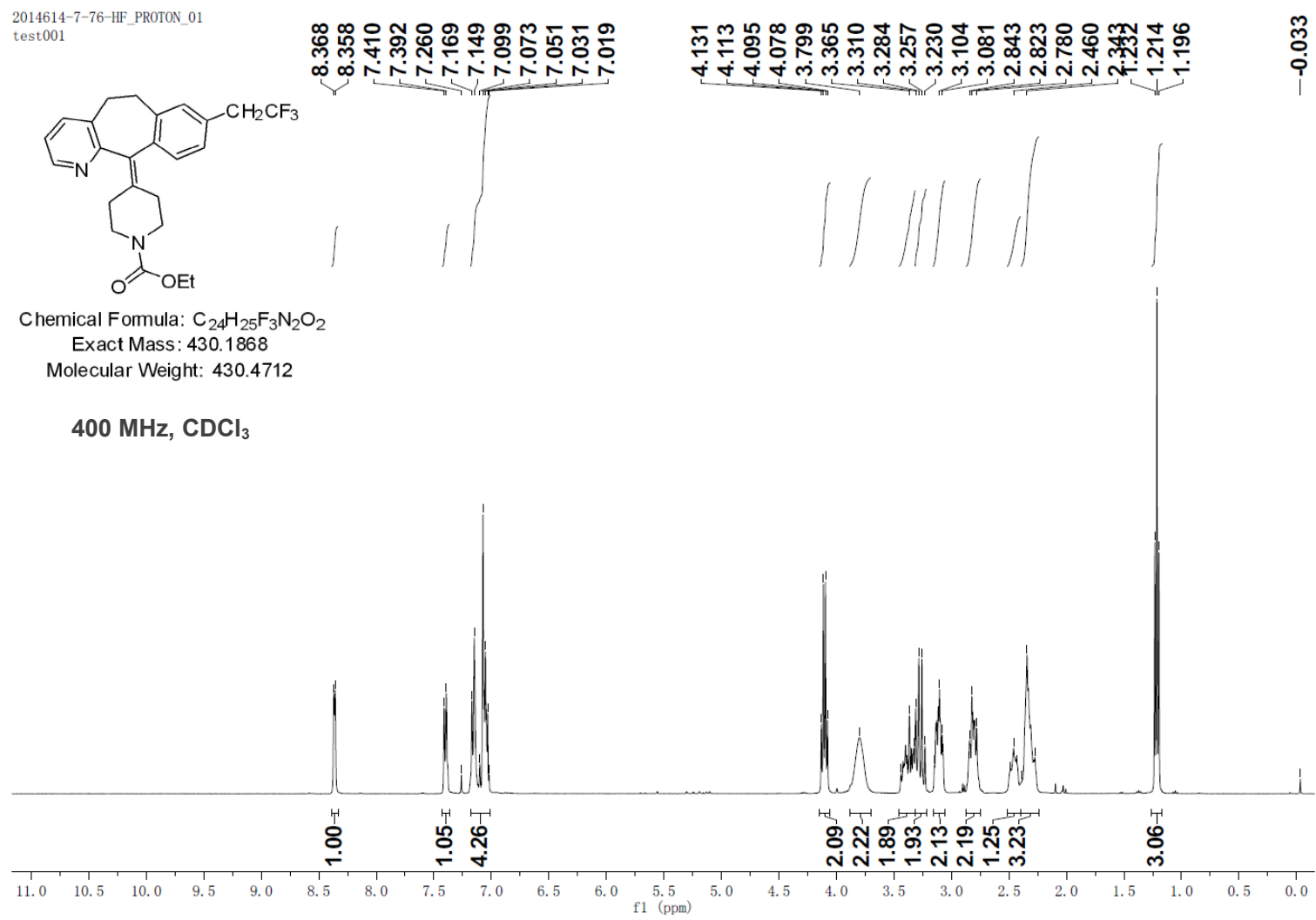




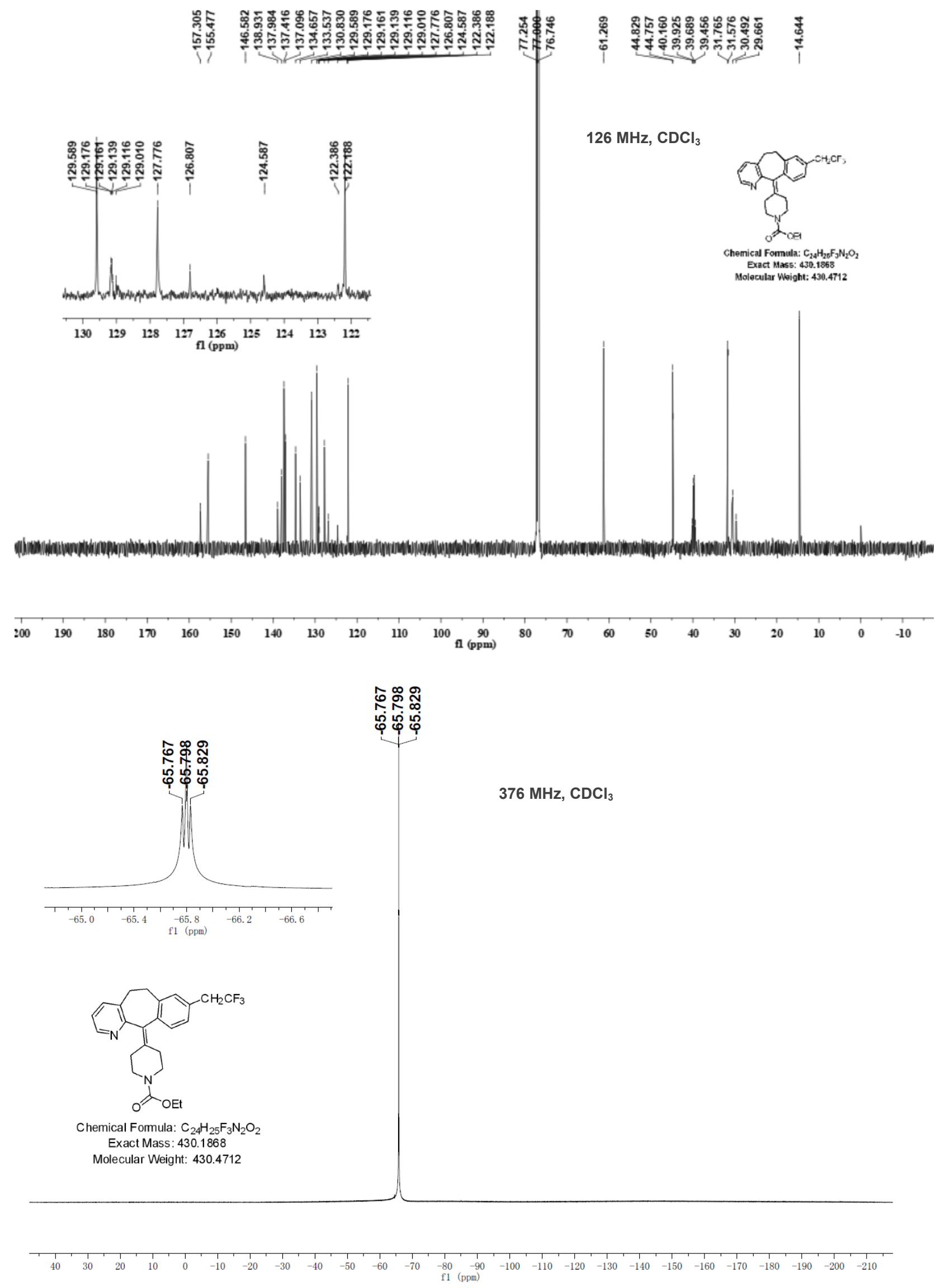



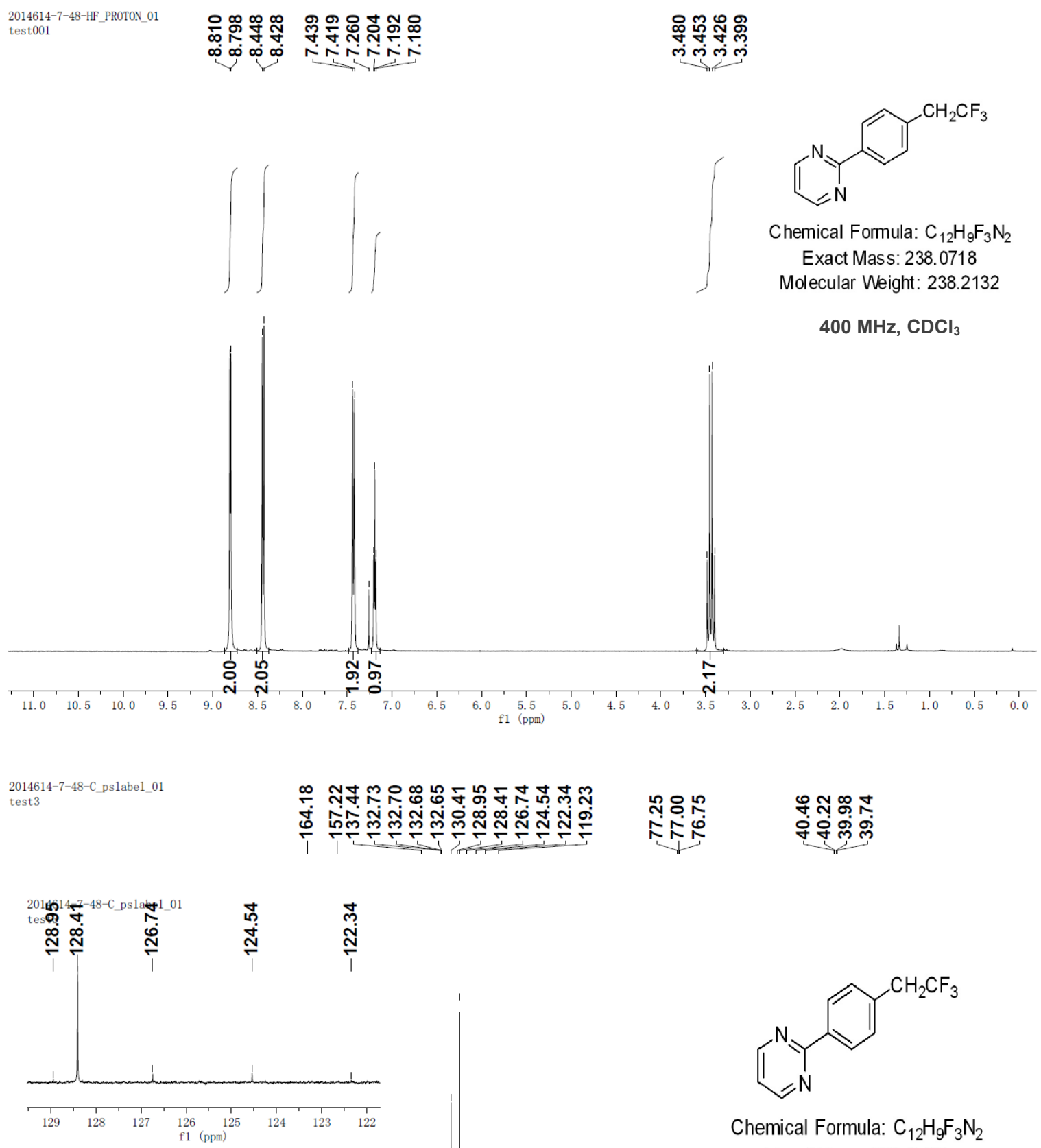

Chemical Formula: $\mathrm{C}_{12} \mathrm{H}_{9} \mathrm{~F}_{3} \mathrm{~N}_{2}$ Exact Mass: 238.0718 Molecular Weight: 238.2132

$126 \mathrm{MHz}, \mathrm{CDCl}_{3}$

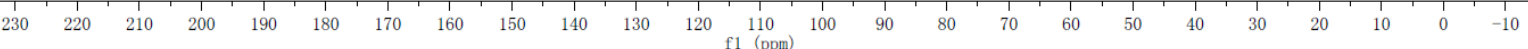


2014614-7-48-HF_FLUORINE_01

test001
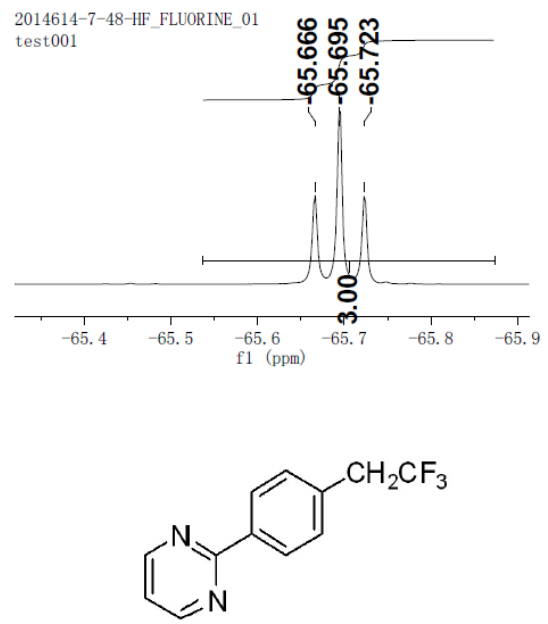

Chemical Formula: $\mathrm{C}_{12} \mathrm{H}_{9} \mathrm{~F}_{3} \mathrm{~N}_{2}$

Exact Mass: 238.0718

Molecular Weight: 238.2132

$376 \mathrm{MHz}, \mathrm{CDCl}_{3}$

Chemical Formula: $\mathrm{C}_{11} \mathrm{H}_{9} \mathrm{~F}_{3} \mathrm{~N}_{2}$

Exact Mass: 226.0718

Molecular Weight: 226.2022

$400 \mathrm{MHz}, \mathrm{CDCl}_{3}$
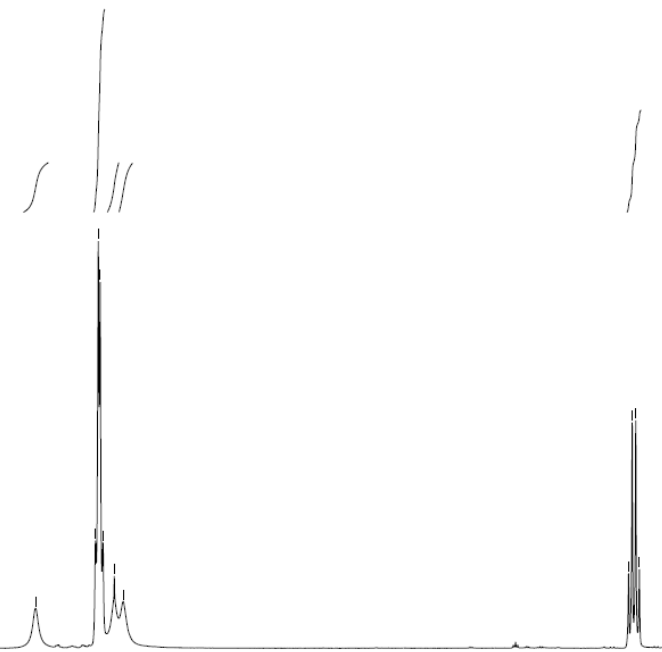

우 8

乌ิ

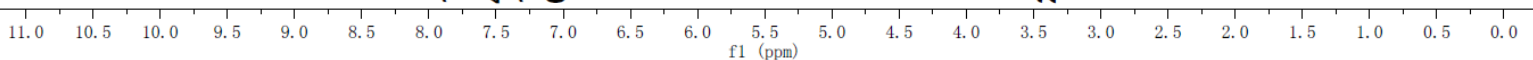




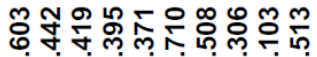

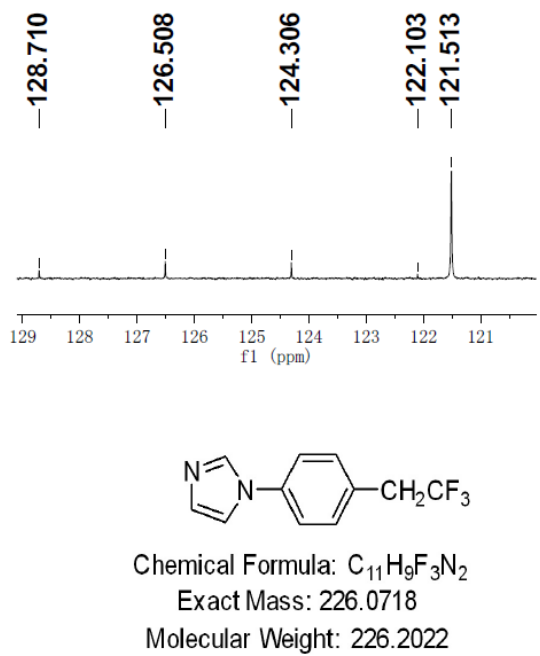
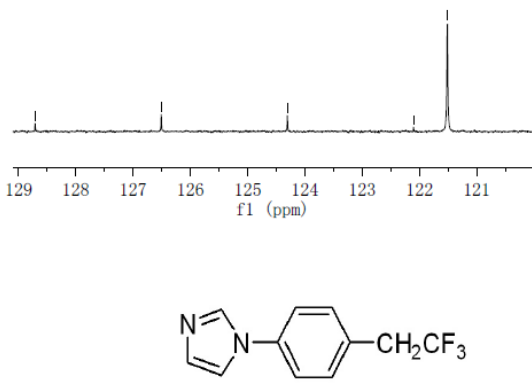$$
126 \mathrm{MHz}, \mathrm{CDCl}_{3}
$$

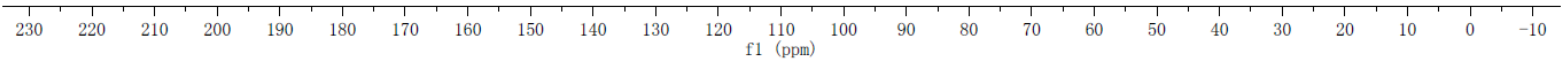

\section{๑ัญ}

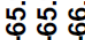

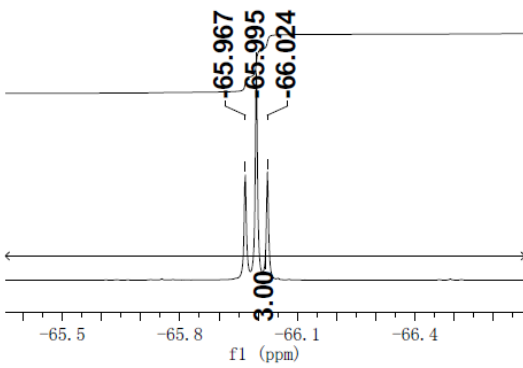

$376 \mathrm{MHz}, \mathrm{CDCl}_{3}$

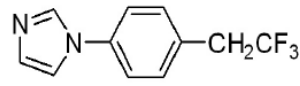

Chemical Formula: $\mathrm{C}_{11} \mathrm{H}_{9} \mathrm{~F}_{3} \mathrm{~N}_{2}$

Exact Mass: 226.0718

Molecular Weight: 226.2022

\section{8}

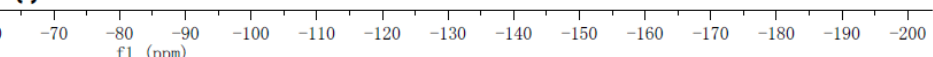




\section{4-(3, 3, 3-Trifluoropropyl)-1, 2-dihydronaphthalene (42)}

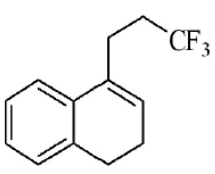

Chemical Formula: $\mathrm{C}_{13} \mathrm{H}_{13} \mathrm{~F}_{3}$

Exact Mass: 226.0969

Molecular Weight: 226.2422

$400 \mathrm{MHz}, \mathrm{CDCl}_{3}$

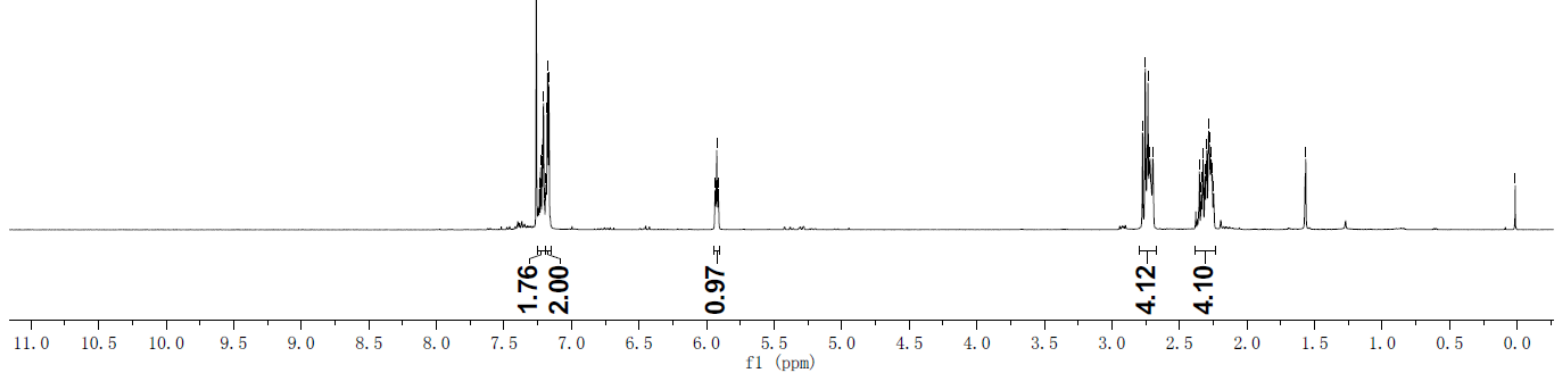

$-7-27-1 \mathrm{C}$

new experiment

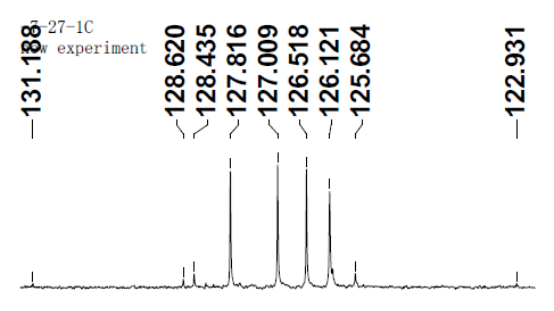

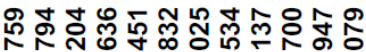

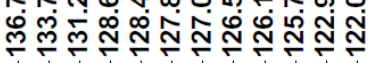

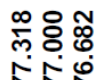

Fíg

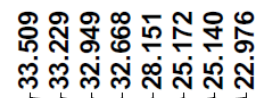

స్

$100 \mathrm{MHz}, \mathrm{CDCl}_{3}$ 
2014614-7-27-1HF_FLUORINE_01

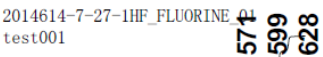

\section{फ़ं}
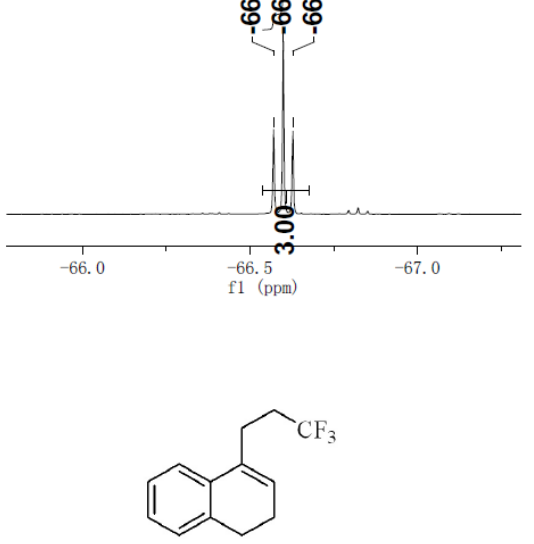

Chemical Formula: $\mathrm{C}_{13} \mathrm{H}_{13} \mathrm{~F}_{3}$ Exact Mass: 226.0969

Molecular Weight: 226.2422

\section{둥욤융}

فํ

$376 \mathrm{MHz}, \mathrm{CDCl}_{3}$

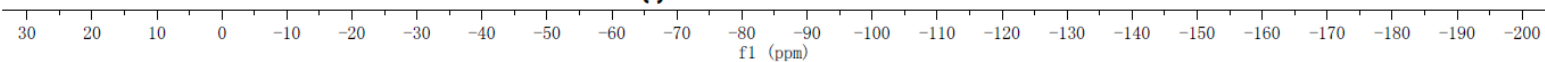

